\title{
Thermal properties of slowly rotating asteroids: results from a targeted survey ${ }^{\star}$
}

\author{
A. Marciniak ${ }^{1}$, V. Alí-Lagoa ${ }^{2}$, T. G. Müller ${ }^{2}$, R. Szakáts ${ }^{3}$, L. Molnár ${ }^{3,4}$, A. Pál ${ }^{3,5}$, E. Podlewska-Gaca ${ }^{1,6}$, N. Parley $^{7}$, \\ P. Antonini ${ }^{8}$, E. Barbotin ${ }^{9}$, R. Behrend ${ }^{10}$, L. Bernasconi ${ }^{11}$, M. Butkiewicz-Bakk ${ }^{1}$, R. Crippa ${ }^{12}$, R. Duffard ${ }^{13}$, \\ R. Ditteon ${ }^{14}$, M. Feuerbach ${ }^{10}$, S. Fauvaud ${ }^{15}$, J. Garlitz ${ }^{16}$, S. Geier ${ }^{17,18}$, R. Goncalves ${ }^{19}$, J. Grice ${ }^{20}$, I. Grześkowiak ${ }^{1}$, \\ R. Hirsch ${ }^{1}$, J. Horbowicz ${ }^{1}$, K. Kamiński ${ }^{1}$, M. K. Kamińska ${ }^{1}$, D.-H. Kim ${ }^{21,22}$, M.-J. Kim² ${ }^{22}$, I. Konstanciak ${ }^{1}$, \\ V. Kudak ${ }^{23,24}$, P. Kulczak ${ }^{1}$, J. L. Maestre ${ }^{25}$, F. Manzini ${ }^{12}$, S. Marks ${ }^{10}$, F. Monteiro ${ }^{26}$, W. Ogłoza ${ }^{27}$, D. Oszkiewicz ${ }^{1}$, \\ F. Pilcher ${ }^{28}$, V. Perig ${ }^{24}$, T. Polakis ${ }^{29}$, M. Polińska ${ }^{1}$, R. Roy ${ }^{30}$, J. J. Sanabria ${ }^{17}$, T. Santana-Ros ${ }^{1}$, B. Skiff ${ }^{31}$, \\ J. Skrzypek ${ }^{1}$, K. Sobkowiak ${ }^{1}$, E. Sonbas ${ }^{32}$, O. Thizy ${ }^{10}$, P. Trela ${ }^{1}$, S. Urakawa ${ }^{33}$, M. Żejmo ${ }^{34}$, and K. Żukowski ${ }^{1}$
}

(Affiliations can be found after the references)

Received 25 January 2019 / Accepted 9 April 2019

\begin{abstract}
Context. Earlier work suggests that slowly rotating asteroids should have higher thermal inertias than faster rotators because the heat wave penetrates deeper into the subsurface. However, thermal inertias have been determined mainly for fast rotators due to selection effects in the available photometry used to obtain shape models required for thermophysical modelling (TPM).

Aims. Our aims are to mitigate these selection effects by producing shape models of slow rotators, to scale them and compute their thermal inertia with TPM, and to verify whether thermal inertia increases with the rotation period.

Methods. To decrease the bias against slow rotators, we conducted a photometric observing campaign of main-belt asteroids with periods longer than $12 \mathrm{~h}$, from multiple stations worldwide, adding in some cases data from WISE and Kepler space telescopes. For spin and shape reconstruction we used the lightcurve inversion method, and to derive thermal inertias we applied a thermophysical model to fit available infrared data from IRAS, AKARI, and WISE.

Results. We present new models of 11 slow rotators that provide a good fit to the thermal data. In two cases, the TPM analysis showed a clear preference for one of the two possible mirror solutions. We derived the diameters and albedos of our targets in addition to their thermal inertias, which ranged between $3_{-3}^{+33}$ and $45_{-30}^{+60} \mathrm{~J} \mathrm{~m}^{-2} \mathrm{~s}^{-1 / 2} \mathrm{~K}^{-1}$.

Conclusions. Together with our previous work, we have analysed 16 slow rotators from our dense survey with sizes between 30 and $150 \mathrm{~km}$. The current sample thermal inertias vary widely, which does not confirm the earlier suggestion that slower rotators have higher thermal inertias.
\end{abstract}

Key words. minor planets, asteroids: general - techniques: photometric - radiation mechanisms: thermal

\section{Introduction}

Thermal infrared flux from asteroids carries information on their surface regolith properties like thermal inertia, surface roughness, regolith grain size, and their compactness. Thermal data also allow us to precisely determine the size and albedo of these objects, when coupled with visual absolute magnitudes. Asteroid sizes are otherwise hard to determine, unless multi-chord stellar occultations, adaptive optics images, or radar echoes are available (Ďrech et al. 2015), yet they are essential characteristics for studies of for example the collisional evolution based on currently observed size-frequency distribution (Bottke et al. 2005), asteroid densities (Carry 2012), or asteroid family members dispersion under thermal recoil force (Vokrouhlický et al. 2015). Physical properties of the regolith covering asteroid surfaces are connected to their age and composition. Young, fresh surfaces display high thermal inertia, because of the small amount of finegrained regolith on the surface, while the surfaces of old targets

\footnotetext{
${ }^{\star}$ Full Table A.1 and photometric data from all individual nights are only available at the CDS via anonymous ftp to cdsarc.u-strasbg.fr $(130.79 .128 .5)$ or via http://cdsarc. u-strasbg.fr/viz-bin/qcat?J/A+A/625/A139
}

are covered with thick layer of insulating regolith, resulting in small thermal inertia values. Also, bodies larger than $100 \mathrm{~km}$ in diameter are usually covered with very fine-grained regolith (thus displaying smaller thermal inertia), while smaller sized ones display signatures of coarser grains in the surface, resulting in larger thermal inertia (Gundlach \& Blum 2013).

These properties can provide valuable insights into the dynamical, collisional and thermal history of asteroids, some of which are intact leftover planetesimals that created planets and shaped the solar system. It might also be possible to study deeper layers under the immediate surface by using longer wavelengths that penetrate deeper, or by studying targets with long episodes of the Sun heating the same surface area, like slow rotators or targets with low spin axis inclination to the orbit.

For detailed studies of asteroid thermal properties, various thermophysical models (henceforth: TPM) are being used (for their overview see Delbo' et al. 2015). The essential input to apply these models is the knowledge of the spin and shape of the objects under study. The rotation period and spin axis inclination dictate the duration and intensity of the alternating cycles of surface heating and cooling. Such cycles, when especially intense, can result in thermal cracking (Delbo' et al. 2014). As 
for the shape, at first approximation it can be represented by a sphere, although such a simple shape often fails to explain thermal lightcurves or separate thermal measurements taken at different viewing aspects and phase angles.

Today the availability of spin and shape modelled targets is the main limiting factor for asteroid studies by TPM. Also, in spite of an abundance of thermal data available mainly from WISE (Wide-field Infrared Survey Explorer), AKARI, IRAS (the Infrared Astronomical Satellite), or Herschel space observatories, they also possess their limitations: for unique solutions from thermophysical modelling, thermal data have to come from a range of aspect and phase angles, probing the target rotation with sufficient resolution at the same time. As a result, so far detailed TPM have been applied to less than 200 asteroids (see the compilations by Delbo' et al. 2015; Hanuš et al. 2018a), and only a small fraction of them rotate slowly, with periods exceeding $12 \mathrm{~h}$. Slow rotators are especially interesting in this context, as a trend of increasing thermal inertia with period has been found by Harris \& Drube (2016), based on estimated beaming parameters $(\eta)$ determined on data from the WISE space telescope (Wright et al. 2010).

The distribution of thermal inertia amongst the members of asteroid families would be crucial for example in the search for evidence for asteroid differentiation, separating iron rich from iron poor family members (Matter et al. 2013). This quantity is also essential for estimates of the orbital drift caused by the Yarkovsky effect, and for studies of regolith properties. However, the relatively small number of targets with known spin and shape parameters prevents detailed thermophysical studies on large groups of asteroids, limiting these to using simple thermal models with spherical shape approximation, often insufficient to explain thermal data taken in various bands and viewing geometries. It is especially vital in the light of high precision thermal infrared data from the WISE and Spitzer space telescopes now available, with uncertainties comparable to average uncertainties of the shape models based on lightcurve inversion (Delbo' et al. 2015; Hanuš et al. 2015).

Nowadays asteroid spin and shape models are created mainly from sparse-in-time visual data (of the order of one to two points per day) from large surveys like those compiled in the Lowell photometric database (Hanuš et al. 2011). The low photometric precision of such data (average $\sigma \sim 0.15-0.2 \mathrm{mag}$ ), originally gathered for astrometric purposes, results in the favouring of some targets in the modelling, for example mainly those displaying large amplitudes in each apparition (Durech et al. 2016). A partial solution to the problem is joining sparse data with dense lightcurves (like in e.g. Hanuš et al. 2013), an approach limited though by the availability of the latter, which are also strongly biased towards large amplitudes and short periods. On the other hand, targeted surveys gathering dense lightcurves favour certain types of objects, like near Earth asteroids (NEAs) or members of specific groups or families like Flora, Eos, or Hungaria asteroids. The resulting spin and shape determinations, even if taken altogether, do not provide unbiased information on for example the spin axis distributions of the whole asteroid population. Instead, they are biased by spin clusters within families (Slivan 2002; Kryszczyńska 2013) and by preferential retrograde rotation of NEAs (Vokrouhlický et al. 2015). Taking all this together can distort the results for the whole population, missing certain specific cases, so that many elements essential for properly understanding asteroid dynamics, physics, and evolution are lacking (Warner \& Harris 2011). For example, Jupiter Trojans on average rotate much more slowly than main belt asteroids, so there might be a gradation of rotation periods with growing heliocentric distance (Marzari et al. 2011). Also, as has been stressed by Warner \& Harris (2011), as much as $40 \%$ of lightcurves considered in the Lightcurve Database $(\mathrm{LCDB})^{1}$ as reliable, have small maximum amplitude: $a_{\max } \leq$ $0.2 \mathrm{mag}$, posing additional challenges in period determination due to potential ambiguities, and having profound effects on the results from any survey using asparse-in-time observing cadence.

Some light on the subject of biases in asteroid models was shed by recent results based on data for asteroids from Gaia Data Release 2 (DR2), which facilitated unique determination of spin parameters for around 200 targets (Ďurech \& Hanuš 2018). This first, largely unbiased sample of targets (with some exceptions, see Santana-Ros et al. 2015) modelled using very precise absolute brightnesses revealed interesting results. Half of the asteroids in this sample turned out to rotate slowly, with periods longer than $12 \mathrm{~h}$, unlike in the sets of models from the majority of previous surveys, based on ground-based data, where shorter periods dominated (e.g. Hanuš et al. 2011, 2013). Results from Gaia DR2 confirm the findings of Szabó et al. (2016) and Molnár et al. (2018) based on data from the Kepler Space Telescope of the substantial contribution of slow rotators in the asteroid population.

It should also be noted that models based on sparse data provide reliable spin parameters, but only low-resolution, coarse shape representations (flag " 1 " or " 2 " in the shape quality system proposed by Hanuš et al. 2018b), limiting their use in other applications (Hanuš et al. 2016). For example, detailed thermophysical modelling needs rather high-resolution (flag “ 3 ”) shape models as input, only possible with large datasets of dense lightcurves obtained at various viewing geometries. Such models usually provide a much better fit to thermal data than shapes approximated by a sphere with the same spin axis and period (Marciniak et al. 2018).

In the next section we refer to our targeted survey, then in Sect. 3 we describe our modelling methods. Results from both lightcurve inversion and thermophysical modelling are presented in Sect. 4, and discussion and conclusions in Sect. 5. Appendix A contains details of the observing runs and composite lightcurves, while Appendix B presents $\mathrm{O}-\mathrm{C}$ plots from thermophysical analysis.

\section{Targeted survey}

Taking all the above-mentioned facts into account, it is clear that there is a continuing need for targeted, dense-in-time observations of objects omitted by previous and ongoing surveys. Such targets are mainly slow rotators, and also bodies that either constantly or temporarily display lightcurves of small amplitudes. To counteract both selection effects, our observing campaign is targeted at main-belt slow rotators $(P>12 \mathrm{~h})$, which at the same time have small maximum amplitudes $\left(a_{\max } \leq 0.25 \mathrm{mag}\right)$. Neither sparse data from large surveys nor available datasets of dense lightcurves allow for their precise spin and shape modelling, so they require a dedicated observing campaign. It is worth noting that it is exactly these features small and slow flux variations - that make them challenging for spin and shape reconstructions, which make these targets perfect calibration sources for a range of infrared observatories (like ALMA - Atacama Large Millimeter Array, APEX - Atacama Pathfinder Experiment, or IRAM - Institut de Radioastronomie Millimétrique, Müller \& Lagerros 2002), on the condition that

http://www. MinorPlanet. info/lightcurvedatabase.html 
these variations can be exactly predicted. To this purpose their reliable spin and shape model are necessary.

The details of our campaign conducted at over 20 stations from 12 countries worldwide, the target selection procedure, and first results are described in Marciniak et al. (2015, 2016). In a nutshell, our survey revealed a substantial number of slow rotators which, in spite of having good data from dense observations, had wrongly determined rotation periods, summarised in LCDB (Warner et al. 2009), a database used in a variety of further studies on asteroid spins and dynamics. We corrected those determinations with the new values for the periods, and confirmed them in consecutive apparitions. We also constructed spin and shape models for the first sample our targets, using dense lightcurves from multiple apparitions, and scaled these models by stellar occultations fitting and thermophysical modelling, with consistent size determinations (Marciniak et al. 2018) In the latter work we also presented simultaneous fits to data from three different infrared missions (IRAS, AKARI, and WISE), and in spite of potential calibration problems of each of the missions alone and cross-calibrations between them, we obtained good fits. This gave us confidence in our models and methods used.

The most important of our previous results presented in Marciniak et al. (2018) were large thermal inertia values obtained for most of our slow rotators, which followed the trend found by Harris \& Drube (2016). This seemed to support the idea that for slow rotators we observe thermal emission from deeper, more compact layers of the surface, a fact that might open new paths for studies of asteroid regolith. However, due to the small number of models for slowly rotating asteroids, until recently thermal inertia values from detailed TPM have been determined for only two such asteroids (227 Elvira and 956 Elisa, Delbo' \& Tanga 2009; Lim et al. 2011; see Fig. 5 in Harris \& Drube 2016; based on compilation by Delbo' et al. 2015, reproduced here in Fig. 12) The above-mentioned trend was found on the values derived from estimated beaming parameters using a simplified approach. Recent results from TPM by Hanuš et al. (2018a) showed a rather large diversity of thermal inertia values for slow rotators within the size range of $10-100 \mathrm{~km}$. Here we further investigate this issue by studying more slow rotators in both visible light and thermal infrared radiation.

Our photometric campaign is ongoing, and new observing stations have joined. Our data sources now also include observations from the Kepler Space Telescope in its extended mission, K2 (Howell et al. 2014). Kepler could not track moving targets during a campaign, so solar system objects could be observed either by using previously allocated, curved, or boomerangshaped masks in accordance with the expected positions of the target minor bodies (see e.g. Pál et al. 2015) or larger continuous pixel masks (so-called supermasks) where such objects also appeared for several days, but not on purpose (see e.g. Molnár et al. 2018). Data processing has been safely established for these observations, regardless of whether these were targeted or not. This processing is based on the registration of the images (to correct for spacecraft jitter) followed by a differential-image photometry by involving oblong-shaped apertures (see Pál et al. 2015; Szabó et al. 2017 and Molnár et al. 2018 for further details). The model of (100) Hekate presented here has been partially based on Kepler data. The asteroid (100) Hekate was observed with a dedicated set of masks for 5.5 and 4.5 days continuously in Campaigns 16 and 18, respectively. Table A.1 gives details of each observing run obtained within the present work. Scarce literature data had to be complemented with from $\sim 100$ up to $\sim 500$ $\mathrm{h}$ of new observations for each target before unique models were feasible.

\section{Lightcurve inversion and thermophysical modelling}

For spin and shape reconstructions we use lightcurve inversion by Kaasalainen \& Torppa (2001), which represents the shape model by a convex polyhedron. All the lightcurves were treated as relative, and not absolutely calibrated, as from our campaign we obtain mostly relative brightness measurements. Only the models with a unique solution, clearly the best in terms of $\chi^{2} \mathrm{fit}$ of observed to modelled lightcurves, have been accepted. Each of the acceptable shape model solutions has been visually inspected to fulfil the criterion of rotation around the axis of greatest inertia. From the range of accepted solutions the uncertainty range of spin axis position and period has been evaluated. As shown by Kaasalainen et al. (2001) this is the only practical way of obtaining realistic uncertainty for spin axis position because formal errors like those obtained from the covariance matrix tend to be strongly underestimated. The effects of random noise are much smaller than systematic errors not uncommon in photometric data, or the combined effects of model shape and spin axis uncertainties. The uncertainty of period is also determined from the range of best-fit solutions, and is dictated by the time span of the whole photometric dataset and the period duration itself. If the minimum in the periodogram is substantially lower than the others, the period uncertainty can be assumed to be one hundreth of this minimum width, which is usually of the same order as the one determined from the best-fit solutions.

As is usually the case when models are based exclusively on dense lightcurves, the uncertainty of the spin axis position (pole) was of the order of a few degrees (see Table 1), and the shape models had a smooth appearance. Formally large uncertainty in some values of pole longitude $\left(\lambda_{\mathrm{p}}\right)$ is due to high inclination of the pole $\left(\beta_{\mathrm{p}}\right)$, translating actually to a relatively small distance on the celestial sphere.

In thermophysical modelling, the diameter $(D)$ and the thermal inertia $(\Gamma)$ are fitted and different surface roughness are tried by varying the opening angle of hemispherical craters covering 0.6 of the area of the facets (following Lagerros 1996), covering rms values between 0.2 and $\sim 1$. Heat diffusion is $1 \mathrm{D}$ and we use the Lagerros approximation (Lagerros 1996, 1998; Müller \& Lagerros 1998; Müller 2002). The TPM implementation is the one used in Alí-Lagoa et al. (2014), based on that of Delbo' \& Harris (2002), however the colour correction is treated differently: instead of colour correcting each facet's flux based on its effective temperature, we now use the $H$ and $G$ values tabulated in the JPL (Jet Propulsion Laboratory) Horizons database to compute an effective temperature based on the heliocentric distance at which each observation was taken (following the approach in Usui et al. 2011). Another simplifying assumption is that spectral emissivity is constant and equal to 0.9 (see e.g. Delbo' et al. 2015), which does not seem unreasonable given the small spectral contrast of $1-3 \%$ found by Licandro et al. (2012) in the $10-\mu \mathrm{m}$ features of $50-60 \mathrm{~km}$ Themis family members (the $20-\mu$ emission plateau is expected to be flatter). Once we obtain $D$, we compute the visible geometric albedo using the $H-G_{12}$ values from Oszkiewicz et al. (2011). The Bond albedo that would be derived from these values is sometimes different from the Bond albedo value used as input for the TPM $\left(A_{i}\right)$. This value was obtained by first averaging the tabulated diameter from IRAS, AKARI, and WISE (Tedesco et al. 2005; Usui et al. 2011; Alí-Lagoa et al. 2018; Mainzer et al. 2016) and occultations (compiled by Dunham et al. 2016). Then, we used this size to compute the Bond albedo from all available $H-G$, $H-G_{12}$, and/or $H-G_{1}-G_{2}$ values from the Minor Planet Center 
Table 1. Spin parameters of asteroid models obtained in this work, with their uncertainty values.

\begin{tabular}{|c|c|c|c|c|c|c|c|c|}
\hline \multirow{2}{*}{$\begin{array}{r}\text { Sidereal } \\
\text { period }(\mathrm{h})\end{array}$} & \multicolumn{2}{|c|}{ Pole 1} & \multicolumn{2}{|c|}{ Pole 2} & \multirow{2}{*}{$\begin{array}{l}\text { rmsd } \\
\text { (mag) }\end{array}$} & \multirow{2}{*}{$\begin{array}{c}\text { Observing span } \\
(\mathrm{yr})\end{array}$} & \multirow[t]{2}{*}{$N_{\text {app }}$} & \multirow[t]{2}{*}{$N_{\mathrm{lc}}$} \\
\hline & $\lambda_{\mathrm{p}}$ & $\beta_{\mathrm{p}}$ & $\lambda_{\mathrm{p}}$ & $\beta_{\mathrm{p}}$ & & & & \\
\hline $\begin{array}{r}\text { (100) Hekate } \\
27.07027 \\
\pm 0.00006\end{array}$ & $\begin{array}{r}104^{\circ} \\
\pm 7^{\circ}\end{array}$ & $\begin{array}{r}+51^{\circ} \\
\pm 2^{\circ}\end{array}$ & $\begin{array}{r}306^{\circ} \\
\pm 8^{\circ}\end{array}$ & $\begin{array}{l}+52^{\circ} \\
\pm 3^{\circ}\end{array}$ & 0.012 & $1977-2018$ & 8 & 62 \\
\hline $\begin{array}{r}\text { (109) Felicitas } \\
13.190550 \\
\pm 0.000004\end{array}$ & $\begin{array}{l}77^{\circ} \\
\pm 1^{\circ}\end{array}$ & $\begin{array}{r}-26^{\circ} \\
\pm 5^{\circ}\end{array}$ & $\begin{array}{r}252^{\circ} \\
\pm 4^{\circ}\end{array}$ & $\begin{array}{l}-49^{\circ} \\
\pm 7^{\circ}\end{array}$ & 0.015 & 1980-2018 & 8 & 62 \\
\hline $\begin{array}{r}\text { (195) Eurykleia } \\
16.52178 \\
\pm 0.00001\end{array}$ & $\begin{array}{r}101^{\circ} \\
\pm 60^{\circ}\end{array}$ & $\begin{array}{l}+71^{\circ} \\
\pm 15^{\circ}\end{array}$ & $\begin{array}{l}352^{\circ} \\
\pm 60^{\circ}\end{array}$ & $\begin{array}{l}+83^{\circ} \\
\pm 15^{\circ}\end{array}$ & 0.015 & 2001-2017 & 7 & 51 \\
\hline $\begin{array}{r}\text { (301) Bavaria } \\
12.24090 \\
\pm 0.00001\end{array}$ & $\begin{array}{l}46^{\circ} \\
\pm 5^{\circ}\end{array}$ & $\begin{array}{r}+61^{\circ} \\
\pm 6^{\circ}\end{array}$ & $\begin{array}{l}226^{\circ} \\
\pm 14^{\circ}\end{array}$ & $\begin{array}{l}+70^{\circ} \\
\pm 6^{\circ}\end{array}$ & 0.014 & 2004-2018 & 5 & 30 \\
\hline $\begin{array}{r}\text { (335) Roberta } \\
12.02713 \\
\pm 0.00003\end{array}$ & $\begin{array}{r}105^{\circ} \\
\pm 7^{\circ}\end{array}$ & $\begin{array}{r}+48^{\circ} \\
\pm 2^{\circ}\end{array}$ & $\begin{array}{r}297^{\circ} \\
\pm 9^{\circ}\end{array}$ & $\begin{array}{l}+54^{\circ} \\
\pm 6^{\circ}\end{array}$ & 0.012 & $1981-2017$ & 9 & 52 \\
\hline $\begin{array}{r}\text { (380) Fiducia } \\
13.71723 \\
\pm 0.00002\end{array}$ & $\begin{array}{l}21^{\circ} \\
\pm 2^{\circ}\end{array}$ & $\begin{array}{r}+34^{\circ} \\
\pm 3^{\circ}\end{array}$ & $\begin{array}{r}202^{\circ} \\
\pm 1^{\circ}\end{array}$ & $\begin{array}{l}+44^{\circ} \\
\pm 2^{\circ}\end{array}$ & 0.016 & 2008-2018 & 6 & 37 \\
\hline $\begin{array}{r}\text { (468) Lina } \\
16.47838 \\
\pm 0.00003\end{array}$ & $\begin{array}{r}74^{\circ} \\
\pm 18^{\circ}\end{array}$ & $\begin{array}{r}+68^{\circ} \\
\pm 6^{\circ}\end{array}$ & $\begin{array}{l}255^{\circ} \\
\pm 17^{\circ}\end{array}$ & $\begin{array}{l}+68^{\circ} \\
\pm 8^{\circ}\end{array}$ & 0.013 & 1977-2018 & 7 & 40 \\
\hline $\begin{array}{r}(538) \text { Friederike } \\
46.739 \\
\pm 0.001\end{array}$ & $\begin{array}{r}168^{\circ} \\
\pm 30^{\circ}\end{array}$ & $\begin{array}{r}-58^{\circ} \\
\pm 5^{\circ}\end{array}$ & $\begin{array}{r}328^{\circ} \\
\pm 25^{\circ}\end{array}$ & $\begin{array}{l}-59^{\circ} \\
\pm 10^{\circ}\end{array}$ & 0.015 & 2003-2018 & 8 & 98 \\
\hline $\begin{array}{r}\text { (653) Berenike } \\
12.48357 \\
\pm 0.00003\end{array}$ & $\begin{array}{r}147^{\circ} \\
\pm 3^{\circ}\end{array}$ & $\begin{array}{r}+\mathbf{1 8}^{\circ} \\
\pm 2^{\circ}\end{array}$ & $\begin{array}{r}335^{\circ} \\
\pm 2^{\circ}\end{array}$ & $\begin{array}{l}+8^{\circ} \\
\pm 1^{\circ}\end{array}$ & 0.009 & 1984-2018 & 6 & 39 \\
\hline $\begin{array}{r}(673) \text { Edda } \\
22.33411 \\
\pm 0.00004\end{array}$ & $\begin{array}{r}66^{\circ} \\
\pm 15^{\circ}\end{array}$ & $\begin{array}{r}+64^{\circ} \\
\pm 6^{\circ}\end{array}$ & $\begin{array}{l}236^{\circ} \\
\pm 13^{\circ}\end{array}$ & $\begin{array}{l}+63^{\circ} \\
\pm 6^{\circ}\end{array}$ & 0.022 & 2005-2017 & 7 & 49 \\
\hline $\begin{array}{r}\text { (834) Burnhamia } \\
13.87594 \\
\pm 0.00002\end{array}$ & $\begin{array}{r}77^{\circ} \\
\pm 10^{\circ}\end{array}$ & $\begin{array}{r}+60^{\circ} \\
\pm 6^{\circ}\end{array}$ & $\begin{array}{r}256^{\circ} \\
\pm 8^{\circ}\end{array}$ & $\begin{array}{l}+69^{\circ} \\
\pm 7^{\circ}\end{array}$ & 0.018 & 2005-2017 & 6 & 32 \\
\hline
\end{tabular}

Notes. The first column gives the sidereal period of rotation, next there are two sets of pole J2000.0 longitude and latitude. The sixth column gives the rms deviations of the model lightcurves from the data, and next follow the photometric dataset parameters (observing span, number of apparitions, and number of individual lightcurve fragments). Pole solutions preferred by TPM are marked in bold.

(Oszkiewicz et al. 2011 or Vereš et al. 2015), and took again the average value. This approach is somewhat arbitrary but the TPM results are not very sensitive to the value of the Bond albedo. Justification for this, further details, and discussion, including information on how we estimate our error bars, are given in Appendix B.

\section{Results}

In this section for each target separately we refer to previous works containing lightcurves, briefly describe our data, the character of brightness variations, and its implications for the spin and shape. Later, the thermal data availability and range are described, followed by the results of applying these models in TPM analysis. In plots like Fig. 1, the fit of model infrared flux variations to thermal lightcurves is presented, and plots like Fig. B.9 show the observation-to-model ratios versus wavelength, helicentric distance, rotational angle, and phase angle.

Table 1 presents spin parameters obtained within this work (sidereal period, spin axis position, and used lightcurve data). Results from thermophysical modelling with our spin and shape models applied are summarised in Table 2, presenting the diameter, albedo, and thermal inertia for the best-fitting model solution. The values obtained here for diameter are "scaling values" of the given spin and shape solutions listed in Table 1 that would otherwise be scale-free. For comparison we also refer there to the diameters obtained previously from the AKARI (Usui et al. 2011), IRAS (Tedesco et al. 2005), and WISE (Mainzer et al. 2011; Masiero et al. 2011) surveys. We also added their taxonomic class following Bus \& Binzel (2002a,b) and Tholen (1989), so that the albedos could be verified for agreement with taxonomy. 
Table 2. Asteroid diameters from AKARI, IRAS, and WISE $\left(D_{\mathrm{A}}, D_{\mathrm{I}}, D_{\mathrm{W}}\right)$ (Tedesco et al. 2005; Usui et al. 2011; Mainzer et al. 2016), compared to our values from TPM (sixth column).

\begin{tabular}{|c|c|c|c|c|c|c|c|c|c|}
\hline \multirow[b]{2}{*}{ Target } & \multirow[b]{2}{*}{$\begin{array}{c}D_{\mathrm{A}} \\
(\mathrm{km})\end{array}$} & \multirow[b]{2}{*}{$\begin{array}{c}D_{\mathrm{I}} \\
(\mathrm{km})\end{array}$} & \multirow[b]{2}{*}{$\begin{array}{c}D_{\mathrm{W}} \\
(\mathrm{km})\end{array}$} & \multirow[b]{2}{*}{$\begin{array}{l}\text { Taxonomic } \\
\text { type }\end{array}$} & \multicolumn{3}{|c|}{ Radiometric solution for combined data } & \multirow[b]{2}{*}{$\begin{array}{l}\mathrm{Rh} \\
(\mathrm{AU})\end{array}$} & \multirow{2}{*}{$\begin{array}{c}\text { Thermal inertia } \\
\text { at } 1 \mathrm{AU} \\
\text { (SI units) }\end{array}$} \\
\hline & & & & & $\begin{array}{l}\text { Diameter } \\
(\mathrm{km})\end{array}$ & $p_{\mathrm{V}}$ & $\begin{array}{l}\text { Thermal inertia } \\
\text { (SI units) }\end{array}$ & & \\
\hline 100 Hekate & 88.52 & 88.66 & 91.421 & $\mathrm{~S}$ & $87_{-4}^{+5}$ & $0.22_{-0.03}^{+0.03}$ & $4_{-2}^{+66}$ & 3.10 & $9_{-5}^{+154}$ \\
\hline 109 Felicitas & 80.81 & 89.44 & 89.000 & $\mathrm{Ch}$ & $85_{-5}^{+7}$ & $0.065_{-0.01}^{+0.008}$ & $40_{-40}^{+100}$ & 3.15 & $95_{-95}^{+236}$ \\
\hline 195 Eurykleia & 89.38 & 85.71 & 80.330 & $\mathrm{Ch}$ & $87_{-9}^{+11}$ & $0.06 \pm 0.02$ & $15_{-15}^{+55}$ & 2.85 & $33_{-33}^{+121}$ \\
\hline 301 Bavaria & 51.90 & 54.32 & 55.490 & $\mathrm{C}$ & $55_{-2}^{+2}$ & $0.047_{-0.003}^{+0.004}$ & $45_{-30}^{+60}$ & 2.65 & $94_{-62}^{+125}$ \\
\hline 335 Roberta & 92.12 & 89.07 & 89.703 & B & $98_{-11}^{+10}$ & $0.046_{-0.008}^{+0.014}$ & Unconstrained & 2.80 & unc. \\
\hline 380 Fiducia & 75.72 & 73.19 & 69.249 & $\mathrm{C}$ & $72_{-5}^{+9}$ & $0.057_{-0.012}^{+0.009}$ & $10_{-10}^{+140}$ & 2.50 & $20_{-20}^{+278}$ \\
\hline 468 Lina & 59.80 & 69.34 & 64.592 & $\mathrm{CPF}$ & $69_{-4}^{+11}$ & $0.052_{-0.014}^{+0.006}$ & $20_{-20}^{+280}$ & 3.00 & $46_{-46}^{+638}$ \\
\hline 538 Friederike & 72.86 & 72.49 & 79.469 & $\mathrm{C}$ & $76_{-2}^{+5}$ & $0.06 \pm 0.01$ & $20_{-20}^{+25}$ & 3.50 & $50_{-50}^{+115}$ \\
\hline 653 Berenike & 46.91 & 39.22 & 56.894 & $\mathrm{~K}$ & $46_{-2}^{+4}$ & $0.18_{-0.03}^{+0.02}$ & $40_{-40}^{+120}$ & 3.00 & $91_{-91}^{+273}$ \\
\hline 673 Edda & 39.38 & 37.53 & 41.676 & $\mathrm{~S}$ & $38_{-2}^{+6}$ & $0.13_{-0.05}^{+0.03}$ & $3_{-3}^{+33}$ & 2.82 & $7_{-7}^{+72}$ \\
\hline 834 Burnhamia & 61.44 & 66.65 & 66.151 & GS: & $67_{-6}^{+8}$ & $0.074_{-0.016}^{+0.014}$ & $22_{-20}^{+30}$ & 2.75 & $47_{-43}^{+64}$ \\
\hline
\end{tabular}

Notes. We also provide the corresponding visible geometric albedo $\left(p_{\mathrm{V}}\right)$ as described in Sect. 3. Finally, we tabulate the nominal thermal inertia $(\Gamma)$ and its value normalised at 1 AU using the mid-value $(\mathrm{Rh})$ between the maximum and minimum heliocentric distances spanned by each object's IR data set. To convert $\Gamma$ values to $1 \mathrm{AU}$ we assumed that $\Gamma$ is proportional to $\mathrm{Rh}^{-3 / 4}$ (see the Discussion section). Error bars are $3-\sigma$.

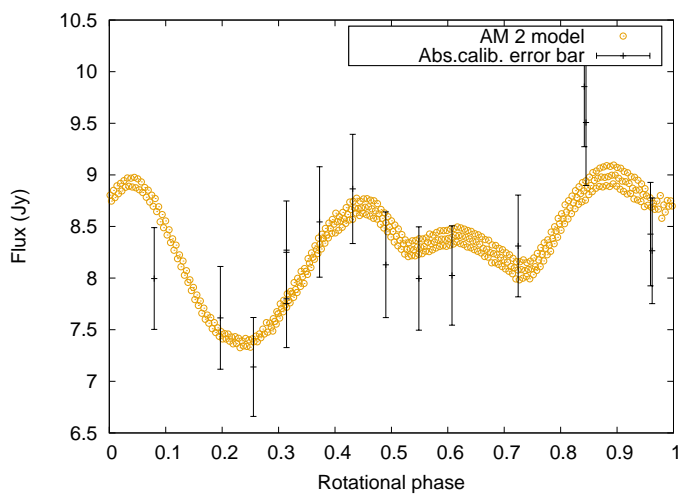

Fig. 1. (100) Hekate W4 data and model thermal lightcurves for shape model 2. Data error bars are $1 \sigma$. Table B.1 summarises the TPM analysis.

For practical reasons, the projections of the shape models and the fit to all the visible lightcurves are not presented here, but will be available from the DAMIT (Database of Astroid Models from Inversion Techniques) $)^{2}$ created by Durech et al. (2010), for models viewing and download, to be used in other applications. In Appendix A (from Figs. A.1 to A.53), we present all the previously unpublished photometric data in the form of composite lightcurves, as they testify the good quality of our spin and shape solutions: photometric accuracy here is mostly at the level of a few millimagnitudes, which is one to two orders of magnitude better than in sparse data standardly used for asteroid modelling. Also, with such slow rotation, separate lightcurves would not show the whole character of the brightness variability, but only a small fraction of the full lightcurve, which is visible only in the folded plots. Moreover, such plots enable us to see lightcurve

2 http://astro.troja.mff.cuni.cz/projects/asteroids3D evolution caused by phase angle effect, which due to various levels of shadowing highlight various shape features. Since we applied no photometric phase correction, their signatures are visible on the overlapping fragments that cover the same rotational phase, but are spaced by more than a month in time like for example in Fig. A.18. Apparitions with only one lightcurve fragment or those with data covering only a small fraction of full rotation, are not presented here, because creating composite lightcurves would be either impossible or would not present much information on the period or the lightcurve appearance. However, such data have been used in the modelling, helping to constrain spin and shape. The full list of observing runs is summarised in Table A.1.

\section{1. (100) Hekate}

We compiled archival lightcurves from four apparitions, published by Tedesco (1979), Gil-Hutton (1990), Hainaut-Rouelle et al. (1995), and Galad et al. (2009), and complemented them with data from the SuperWASP survey (Wide Angle Search for Planets, Grice et al. 2017), and our own data from three more apparitions (see Figs. A.1-A.4). SuperWASP cameras are known to suffer from the detector having a temperature dependence, so the absolute lightcurves can be systematically shifted in magnitude depending on the temperature of the detector. However, as we treat them as relative lightcurves this should not be an issue. In the last apparition, Hekate was observed for our project by the Kepler Space Telescope, resulting in two continuous, fourday-long lightcurves of great quality. The K2 data have been reduced with the fitsh package, using the same methods that were already applied to targeted observations of Trojan asteroids and chance observations of main-belt asteroids (MBAs) in the mission (Pál 2012; Szabó et al. 2016, 2017; Molnár et al. 2018). Overall, Hekate displayed interesting, asymmetric lightcurves of amplitudes ranging from 0.11 to 0.23 magnitude and a long, $27.07 \mathrm{~h}$ period. 
The spin parameters of our model are presented in Table 1. To construct it, initial scanning of parameter space needed an increased number of trial poles and iteration steps. Overall both spin solutions fit the visual lightcurves at a very good level of $0.012 \mathrm{mag}$.

\section{TPM analysis}

The two mirror pole solutions of Hekate are named AM 1 and AM 2. We used 52 infrared observations, 32 from IRAS $(8 \times 12 \mu \mathrm{m}, 8 \times 25 \mu \mathrm{m}, 8 \times 60 \mu \mathrm{m}, 8 \times 100 \mu \mathrm{m})$, five from AKARI $(2 \times$ S9W, $3 \times$ L18W), and 15 from WISE (W4). We assumed $A=0.090$ for the Bond albedo.

Both models provide formally acceptable fits, as do the corresponding spheres (see Table B.1 and the $\chi^{2}$ plots in Fig. B.9). The fit to the WISE "lightcurve" seems reasonable for both models, although not around the zero rotational phases shown in Figs. 1, B.1, and B.9 (third panel from the top). The IRAS data present a slope in the observation-to-model ratios (OMR) versus wavelength plot, which is shown in the top panel of Fig. B.9. Model 2 provides a better fit than 1 and the spheres so we select it as our provisionally favoured solution. Nonetheless, these results could be biased by our neglecting the dependence of thermal conductivity - and therefore thermal inertia - with temperature (see Rozitis et al. 2018, and the review in Delbo' et al. 2015, for instance), over the wide range of heliocentric distances sampled (2.6-3.6 au). However, there is not enough data taken at long heliocentric distances to make a conclusive statement.

To summarise the TPM analysis: the surface roughness is not constrained, the diameter is $87_{-4}^{+5} \mathrm{~km}$, and geometric albedo, $p_{\mathrm{V}}=0.22_{-0.03}^{+0.03}$. We would benefit from additional thermal light curves at negative phase angles (i.e. after opposition) and higher heliocentric distances (to study the conductivity dependence with temperatures). Low thermal inertias of approximately 5 SI units ${ }^{3}$ and low to medium roughness fit the data better.

\section{2. (109) Felicitas}

Available photometric data for Felicitas came from the works of Zappala et al. (1983), Harris \& Young (1989), and from the SuperWASP archive (Grice et al. 2017). We added to these two apparitions data from five more obtained within our campaign, and also data from the WISE satellite obtained in W1 band, which is dominated by reflected light (thermal contribution in $\mathrm{W} 1$ for this target was estimated at $8-30 \%$ ). The latter dataset greatly helped to constrain the model, in an approach first proposed by Ďurech et al. (2018). Felicitas lightcurves have been changing substantially between the apparitions, reaching peakto-peak amplitudes from 0.06 to $0.22 \mathrm{mag}$, with the synodic period around $13.194 \mathrm{~h}$ (Figs. A.6-A.8).

\section{TPM analysis}

The two mirror solutions for Felicitas from Table 1 are denoted AM 1 and AM 2. In the thermal approach we used 38 observations, 15 from IRAS $(5 \times 12 \mu \mathrm{m}, 7 \times 25 \mu \mathrm{m}, 3 \times 60 \mu \mathrm{m})$, five from AKARI $(3 \times \mathrm{S} 9 \mathrm{~W}, 2 \times \mathrm{L} 18 \mathrm{~W})$, and 18 from WISE $(9 \times \mathrm{W} 3$, $9 \times$ W4). We assumed $A=0.025$ for the Bond albedo.

Unlike the AM 2 model, AM 1 provides a formally acceptable fit (Table B.2, and the $\chi^{2}$ plot in Fig. B.10), although the WISE bands are not fitted equally well: the model overestimates the W4 data and underestimates the W3 (top panel in Fig B.10).

\footnotetext{
$3\left(\mathrm{Jm}^{-2} \mathrm{~s}^{-0.5} \mathrm{~K}^{-1}\right)$
}
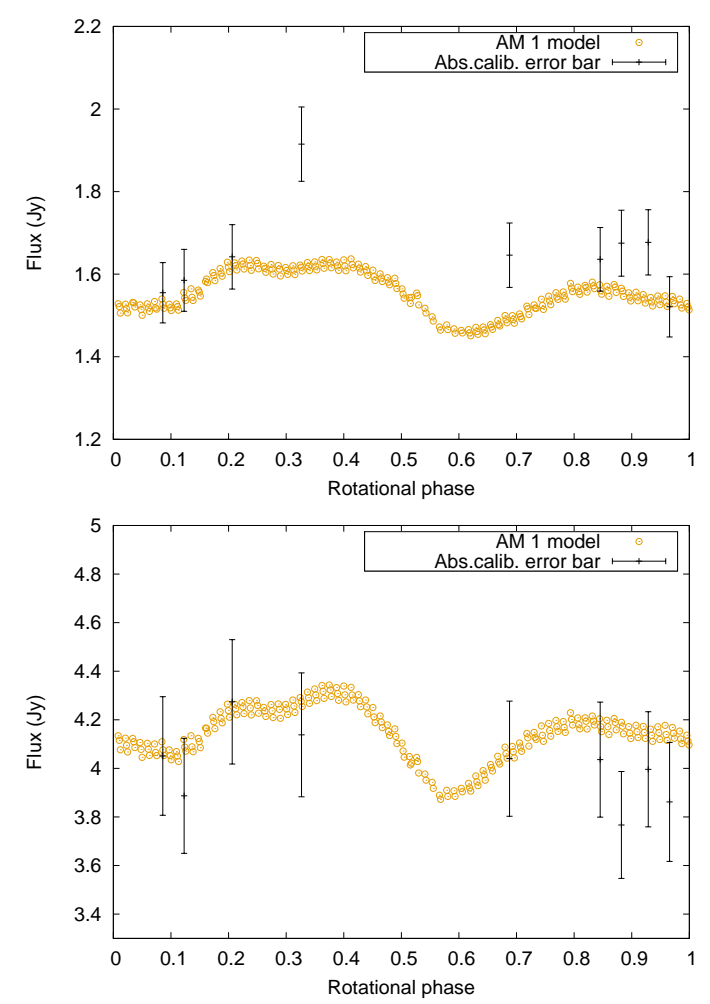

Fig. 2. Asteroid (109) Felicitas W3 (top) and W4 (bottom) data and model thermal light curves for shape model AM 1 (see Table B.2).

Even though the model thermal lightcurves miss some W3 and W4 fluxes (Fig. 2), we consider it as a preliminary approximated solution given that it fits significantly better than the sphere. Additional thermal curves could help confirm or reject this model and data at negative phase angles could improve the constraints for the thermal inertia.

As usual, the surface roughness is not constrained. From the diameter $\left(85_{-5}^{+7} \mathrm{~km}\right)$, we obtain the albedo $p_{\mathrm{V}}=0.065_{-0.01}^{+0.008}$.

\section{3. (195) Eurykleia}

There was no prior publication presenting lightcurves of Eurykleia, but plots from three apparitions are available on the observers' web pages ${ }^{4}$, indicating a long, $16.52 \mathrm{~h}$ period. Here we publish all of these data, adding more recent observations from four more apparitions. During all the seven apparitions, Eurykleia displayed similarly shaped lightcurves with one minimum sharper than the other, which was accompanied by an additional bump or shelf (see Figs. A.9-A.14). The amplitude was stable, being always around 0.24 mag, which indicates a stable aspect angle due to the high inclination of the spin axis. As expected, the resulting model has a high value of $|\beta|$, and a formally large range of possible values of $\lambda$ (see Table 1 ).

\section{TPM analysis}

The two mirror solutions from lightcurve inversion are labelled AM 1 and AM 2. We used a total of 57 infrared observations from IRAS (nine epochs $\times$ four filters), AKARI $(4 \mathrm{~S} 9 \mathrm{~W}+5$ L18W) and WISE W4 (12). The WISE W3 data were saturated for this object and the W4 data, with reported magnitudes

\footnotetext{
4 http://obswww. unige.ch/ behrend/page_cou.html, http://eoni.com/ garlitzj/Period.htm
} 


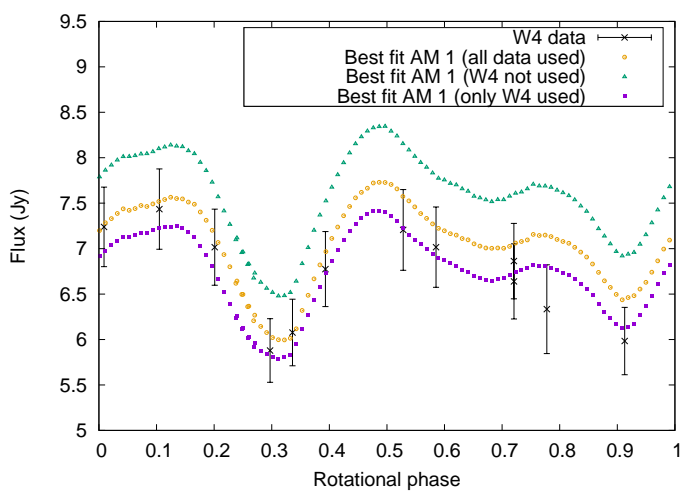

Fig. 3. W4 data and model thermal lightcurves for (195) Eurykleia's shape model AM 1. The different models resulted from fitting different subsets of data. Table B.3 contains the corresponding thermo-physical parameters.

between 0.00 and 0.35 , are near the $-0.6 \mathrm{mag}$ identified as the onset of saturation for individual W4 images (Cutri et al. 2012). We assumed $A=0.020$ for the Bond albedo.

Both shape models provide statistically similarly good fits to all the data, so the TPM cannot reject any of the mirror solutions in this case. Using all the data to optimise the $\chi^{2}$ (i.e. considering $v=57-2=55$ degrees of freedom), the minimum reduced chi-squared $\left(\bar{\chi}_{\mathrm{m}}^{2}\right)$ were 0.51 and 0.60 for the AM 1 and AM 2 models, respectively. These are significantly better than the values obtained for spheres with the same respective spin pole orientation (see Table B.3).

Although the thermal data seems to be fitted very well based on our small $\bar{\chi}_{\mathrm{m}}^{2}$, the OMR of many IRAS data are slightly but systematically above 1 , the WISE OMRs slightly below 1 , and the AKARI ones are not fully horizontally aligned (Fig. B.11, upper panels). Figures 3 and B.2 show that both shape models fit the variation of the W4 thermal lightcurve reasonably well. However, the fluxes predicted by the TPM solution that best fits all the data (yellow open circles) systematically overestimate most of the W4 observations. This is worse for the solution that best fits the AKARI and the IRAS data (open triangles), because the fitted diameter is larger in this case (third line in Table B.3, first and third plot from the top in Fig. B.12). However, the OMR values for the IRAS and AKARI data do align horizontally (Fig. B.11, lower panels). Finally, we can fit the lightcurve much better if we optimise the W4 data alone (filled squares), but the thermal inertia is lower by a factor of three (fourth entry in Table B.3, second and fourth plot in Fig. B.12).

Conclusions from TPM analysis are the following: both shapes seem to fit the data well, including the shape of the W4 thermal lightcurve (Fig. 3), although the flux level of the W4 data is systematically below our fitted model's fluxes. We do not have an explanation for such a systematic mismatch, but perhaps additional thermal IR data could correct the TPM diameter of (195) Eurykleia to a higher value closer to $90 \mathrm{~km}$, or a lower value closer to $80 \mathrm{~km}$. Thus, with the current dataset, the possible diameters range from 78 to $98 \mathrm{~km}$ depending on which subsets of data we fit (Table B.3). As a compromise, we take the midvalue in this range, $87_{-9}^{+11} \mathrm{~km}(3 \sigma$ level error bars, $\sim 13 \%$ relative error).

On the other hand, the large $3 \sigma$ range of possible thermal inertias does not change when we fit different subsets of data, but the error bars are widely asymmetric. We can only provide an upper limit of $70 \mathrm{SIu}$, with a best-fitting value of $\Gamma=15 \mathrm{SIu}$. Surface roughness is not constrained at the $3 \sigma$ level, but high

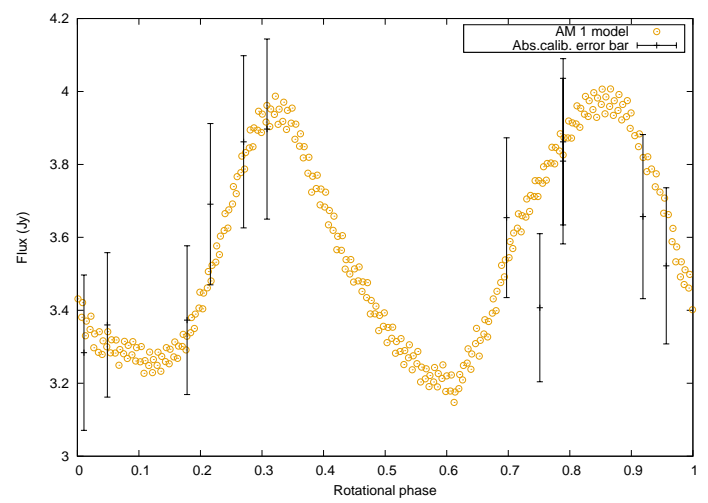

Fig. 4. Asteroid (301) Bavaria AM 1 model thermal lightcurves and W4 data (see Table B.4).

roughness models fit the data better, as is usually the case for main-belt asteroids.

Finally, the geometric albedos derived from our radiometric diameters vary slightly depending on the photometric phase correction applied and the source of the absolute magnitudes and slope parameters. To compute our final value, we use the $H-G_{12}$ values from the Oszkiewicz et al. (2011) table and calculate the maximum and minimum possible $p_{V} \mathrm{~s}$ using all the three sources listed above. We find $p_{\mathrm{V}}=0.06 \pm 0.02$.

\section{4. (301) Bavaria}

The lightcurve from only one previous apparition of asteroid Bavaria has been reported in the literature (Warner 2004). Our campaign built an extensive lightcurve dataset for this target, covering four viewing aspects and a wide range of phase angles. Our data covered sometimes four to five months within one apparition, and less than a year passed between consecutive observed apparitions. Such an observing strategy was defined as the most optimal for spin and shape reconstructions by Slivan (2012), and is confirmed by our experience. Bavaria during its 12.24-h-long rotation displayed wide minima and amplitudes from 0.25 to 0.36 magnitudes (Figs. A.15-A.18), exceeding our initial target selection criteria in the course of the campaign; nonetheless it was retained in the target list. Although the solution for the sidereal period and two possible spin axis positions are very well defined, due to the high value of pole latitude (see Table 1) the shape model vertical extent is poorly constrained.

\section{TPM analysis}

The two mirror solutions from Table 1 are AM 1 and AM 2. We used 36 thermal observations, 18 from IRAS $(9 \times 25 \mu \mathrm{m}$, $9 \times 60 \mu \mathrm{m})$, six from AKARI $(3 \times \mathrm{S} 9 \mathrm{~W}, 3 \times \mathrm{L} 18 \mathrm{~W})$, and 12 from WISE (W4). We rejected IRAS 12- and 100-micron data because they contain clear outliers (by a factor of several). We assumed $A=0.020$ for the Bond albedo.

Both models and the corresponding spheres provide a good fit (see Table B.4, and the $\chi^{2}$ plots in Fig. B.13), although with slightly offset diameters and different roughness (the spheres require low roughness rather than medium). The AM 1 OMR plots are shown in Fig. B.13, and the W4 model thermal lightcurves with the data in Figs. 4 and B.3.

As usual, the roughness is not constrained at the $3 \sigma$ level. With a diameter $55_{-2}^{+2} \mathrm{~km}$, we get $p_{\mathrm{V}}=0.047_{-0.003}^{+0.004}$ and a thermal inertia between 10 and 100 SI units. 


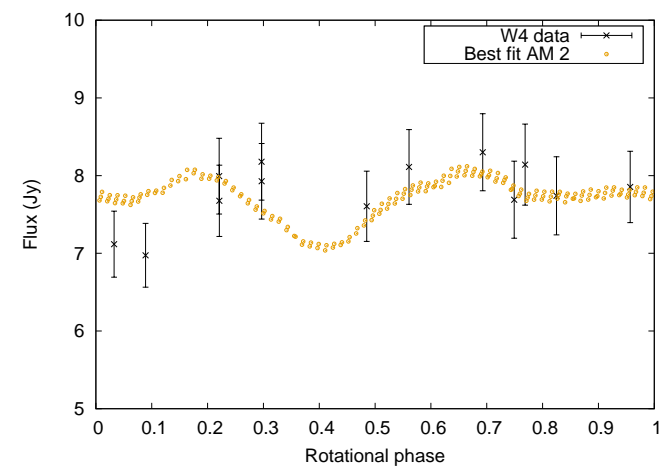

Fig. 5. Asteroid (335) Roberta's WISE data and model thermal lightcurves for shape model 2 and the corresponding best-fitting solutions (very low thermal inertia of $15 \mathrm{SIu}$ ).

\section{5. (335) Roberta}

We compiled a large dataset covering as many as nine apparitions of asteroid Roberta, including data from Binzel (1987), Harris et al. (1992), Lagerkvist et al. (1995), Warner et al. (2007), Pilcher \& Martinez (2015), SuperWASP archive (Grice et al. 2017), and our own data (presented in Figs. A.19-A.21). This target showed rather rare, monomodal lightcurves of amplitudes from 0.13 to $0.19 \mathrm{mag}$, depending on the aspect. Its rotation period, $12.027 \mathrm{~h}$, commensurate with an Earth day, required observations from sites well spaced in longitude for full coverage. Thanks to such an extensive dataset both spin and shape solutions are well constrained (Table 1), however different from those found by Blanco et al. (2000), who reported four pole solutions, all much lower in $|\beta|$ than ours, and a sidereal period longer by $0.027 \mathrm{~h}$, which is a substantial difference.

\section{TPM analysis}

The two mirror solutions are AM 1 and AM 2. Thermal data consisted of 44 observations, 22 from IRAS $(6 \times 12 \mu \mathrm{m}$, $6 \times 25 \mu \mathrm{m}, 6 \times 60 \mu \mathrm{m}, 4 \times 100 \mu \mathrm{m})$, nine from AKARI $(4 \times \mathrm{S} 9 \mathrm{~W}$ and $5 \times \mathrm{L} 18 \mathrm{~W}$ ), and 13 from WISE (W4). We assumed $A=0.020$ for the Bond albedo.

The best solutions for both models are comparably good, and even the spheres provide a formally good fit with compatible results (see Table B.5, and the $\chi^{2}$, and OMR plots in Fig. B.14). The diameter is constrained at the $3 \sigma$-level but with a relatively large relative error of $\sim 10 \%$. The fit to the WISE lightcurve (Figs. 5 and B.4) shows that the fit at phases between 0.0 and 0.10 might be improved with additional visible data for the inversion (phase 0 corresponds to JD 2442489.835789).

The problem with this model is that the $\bar{\chi}_{\text {min }}^{2}$ versus $\Gamma$ curve is very shallow at high $\Gamma$ s, so the thermal inertia is basically unconstrained. The aspect angles sampled by the thermal data are concentrated around equatorial values, so additional data at other sub-observer latitudes and a dense thermal lightcurve at pre-opposition (positive phase angle) would help. The diameter constraint of $98_{-11}^{+10} \mathrm{~km}$ leads to an albedo of $p_{\mathrm{V}}=0.046_{-0.008}^{+0.014}$.

\section{6. (380) Fiducia}

A previous lightcurve of Fiducia was published by Warner (2004). With this work we add six apparitions, confirming the synodic period around $13.72 \mathrm{~h}$. Fiducia's lightcurve shape varied substantially from one apparition to another, reaching 0.32 mag amplitude in one, and then decreasing down to 0.04 in the other (see Figs. A.22-A.28). In the year 2014 Fiducia was observed

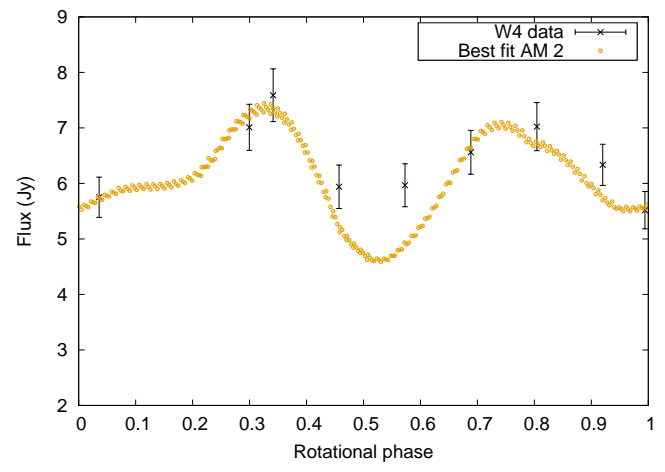

Fig. 6. Asteroid (380) Fiducia's WISE data and model thermal lightcurves for shape model 2 and the corresponding best-fitting solutions (very low thermal inertia of $10 \mathrm{SIu}$; see Table B.6).

exclusively on the $0.8 \mathrm{~m}$ TJO (Telescopi Joan Oró) telescope in the Montsec Observatory in a mode similar to "dense-sparse cadence" described by Warner \& Harris (2011). In spite of the relative sparseness of datapoints, the lightcurve's general character is clearly outlined, and the synodic period from other apparitions is confirmed (Fig. A.24).

In the apparition on the verge of the years 2015 and 2016, Fiducia was observed under an exceptionally large range of phase angles (Table A.1), which resulted in distinctive differences in the lightcurve character. Thus we present data from that apparition on two separate plots, one for small, and the other for large phase angles (Figs. A.26 and A.25, respectively).

The unique solution for the sidereal period could only be found in this case during denser scanning of parameter space. In spite of clear signatures of low inclination of the spin axis, the resulting pole solution is located at rather moderate latitudes (see Table 1). This might be a symptom of the lightcurve inversion method bias against low poles found by Cibulková et al. (2016), as small shape modifications can compensate for the shifted spin axis position of the model.

\section{TPM analysis}

The two obtained mirror solutions are AM 1 and AM 2. We had 54 thermal observations at our disposal, 38 from IRAS $(10 \times 12 \mu \mathrm{m}, 10 \times 25 \mu \mathrm{m}, 10 \times 60 \mu \mathrm{m}, 8 \times 100 \mu \mathrm{m})$, seven from AKARI $(5 \times$ S9W and $2 \times \mathrm{L} 18 \mathrm{~W})$, and nine from WISE (W4). We assumed $A=0.020$ for the Bond albedo.

The best solutions for both models have comparable $\bar{\chi}_{\text {min }}^{2}$ and very shallow minima in the $\bar{\chi}_{\min }^{2}$ versus $\Gamma$ plots. Thermal inertias between 0 and 150 SIu fit the data ( $3 \sigma$ limits), but the best fit is $\Gamma=10$ SI units (see Table B.6, the $\chi^{2}$ plot, and OMR plot in Fig. B.15). The corresponding spheres give similar diameters but fit the data better with higher thermal inertia $(\Gamma \approx 200 \mathrm{SIu})$. Only the most extreme roughness values produce fits outside the $3 \sigma$ range, and low roughness solutions $(\mathrm{rms}<0.30)$ fit the data better. The shapes reasonably reproduce the W4 lightcurve (Figs. 6 and B.5), but the fits could probably be improved with "less boxy" shape models.

From TPM analysis one can conclude that the roughness is unconstrained, but thermal inertias are lower than 150 at the $3 \sigma$ level (the best fit is for $\Gamma=10 \mathrm{SIu}$ ). The diameter range of $72_{-5}^{+9} \mathrm{~km}$ leads to an albedo $p_{\mathrm{V}}=0.057_{-0.012}^{+0.009}$.

\section{7. (468) Lina}

Lightcurves from two previous apparitions of Lina have been published by Tedesco (1979) and Buchheim (2007). Our visual 


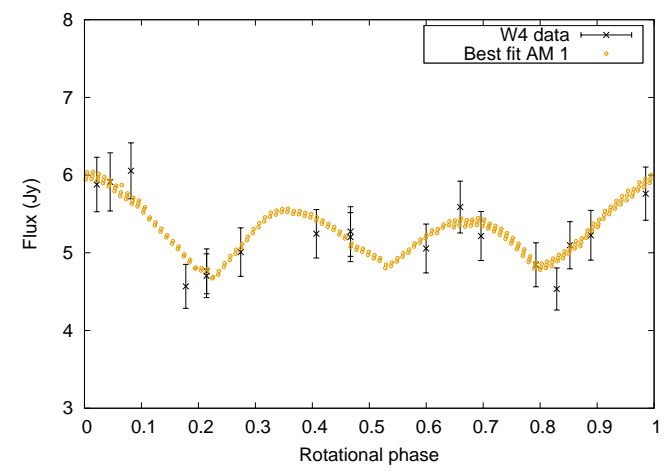

Fig. 7. Asteroid (468) Lina's WISE data and model thermal lightcurves for shape model 1's corresponding best-fitting solutions (see Table B.7).

observations of this target spanned six apparitions, and we found it displaying complex lightcurves, with wide, wavy maxima and narrow minima (Figs. A.29-A.33). Peak-to-peak amplitudes ranged between 0.13 and 0.18 mag within its $16.48 \mathrm{~h}$ period. Model spin parameters are given in Table 1. Shape model stretch along the spin axis is somewhat uncertain.

\section{TPM analysis}

The two mirror solutions are named AM 1 and AM 2. We used 33 observations, seven from IRAS $(2 \times 12 \mu \mathrm{m}, 2 \times 25 \mu \mathrm{m}$, $2 \times 60 \mu \mathrm{m}, 1 \times 100 \mu \mathrm{m})$, eight from AKARI $(2 \times \mathrm{S} 9 \mathrm{~W}$ and $6 \times \mathrm{L} 18 \mathrm{~W}$ ), and 20 from WISE (W4), and assumed $A=0.020$ for the Bond albedo.

The comparatively fewer data points are available for this object. The best-fitting roughness and thermal inertias are low (rms $\sim 0.3$ and $\Gamma=20$ SIu; see Table B.7, and the $\chi^{2}$ plots in Fig. B.16), even for the spherical models. The fit to the WISE lightcurve is reasonably good (Figs. 7 and B.6), but the $\bar{\chi}_{\min }^{2}$ is 1.20 , which is not optimal. The data sample widely different heliocentric distances, from 2.5 to 3.5 au (Fig. B.16), but the residuals do not present a strong trend.

The analysis points to thermal inertias lower than $300 \mathrm{SIu}$ and low roughness, but this object requires more thermal data to constrain these properties better. Even the diameter has a relatively large error bar by TPM standards: $69_{-4}^{+11} \mathrm{~km}$. The corresponding albedo is $0.052_{0.014}^{+0.006}$.

\section{8. (538) Friederike}

Data previously published came from one apparition and clearly displayed a very long, $46.728 \mathrm{~h}$ period for this target (Pilcher 2013). In addition to it we used previously unpublished data from the years 2003 and 2006, with only partial coverage though (Table A.1), and own data from five more apparitions. Those with good phase coverage are shown in Figs. A.34-A.37. Lightcurves of Friederike had minima of different widths and amplitudes from 0.20 to $0.25 \mathrm{mag}$.

In spite of a large and varied dataset, only with the addition of WISE W1 data could the unique solution for period, spin axis, and shape be found. These data proved to be a necessary, continuous basis lasting a few tens of hours, while other lightcurves covered only at most $20 \%$ of the full rotation. Such extremely long period targets are especially challenging for ground-based studies, being better targets for wide-field, space-borne observatories. Due to this long period and relatively short time span of the observational dataset, the precision of the sidereal period determination is lower than in the case of other targets (Table 1).

\section{TPM analysis}

The two mirror spin-shape solutions are AM $1\left(168^{\circ},-58^{\circ}\right.$, $46.73928 \mathrm{~h})$ and AM $2\left(328^{\circ},-59^{\circ}, 46.73985 \mathrm{~h}\right)$. Friederike is well observed at thermal wavelengths: two four-band IRAS measurements, ten measurements by AKARI $(4 \times \mathrm{S} 9 \mathrm{~W}, 6 \times \mathrm{L} 18 \mathrm{~W})$, taken at different epochs before and after opposition, and 23 WISE data points (at W3 and W4), also at two epochs before and after opposition.

Solution AM 1 provides a borderline acceptable fit to all thermal data simultaneously, as do the corresponding spheres (see Table B.8). However, the AM 2 solution seems to match the WISE W3 and W4 lightcurves a bit better. The residuals in the observation-divided-by-model plots (see Fig. B.17) indicate that the spin-shape solutions are not perfect or - alternatively - that there are surface variegations that influence the thermal fluxes. The WISE W3 and W4 lightcurves favour AM 2, but do not help to settle the spin ambiguity completely. Overall, in this case high and extremely high surface roughness worked very well and all the radiometric solutions point to low values for the thermal inertia well below $\Gamma=20$ SIunits (see $\chi^{2}$ plots in Fig. B.17), with a trend to higher inertias at shorter heliocentric distance (2.6 au) and lower values at the largest heliocentric distance $(>3.5 \mathrm{au})$. The heliocentric influence on thermal inertia is also visible in the radiometric solutions for the individual datasets (see Table B.8): all WISE data are taken at $r_{\text {helio }}>3.4$ au, while the AKARI and IRAS data are taken well below 3.0 au. The overall best radiometric solution for AM 2 and extremely high surface roughness produces $\Gamma=20$ SIunits, a diameter of $76_{-2}^{+4}$, and a geometric albedo of $0.06 \pm 0.01$

\section{9. (653) Berenike}

Berenike displayed small amplitudes in the range of $0.05-0.16$ (Figs. A.38-A.41), also in the archival observations by Binzel (1987) and Galad \& Kornos (2008). However, in the last apparition observed in this work the amplitude unexpectedly rose to $0.38 \mathrm{mag}$, being over two times larger than ever, which is confirmed by two independent observing runs (Fig. A.42). That apparition must have provided the only viewing geometry when signatures of full elongation of this target could be visible. This case shows the importance of probing a wide range of geometries for correct reproduction of the spin axis position and shape elongation. The synodic period was around $12.485 \mathrm{~h}$, and the full dataset spanned seven apparitions.

The shape model is indeed elongated in the equatorial dimensions, and this time the pole position is low, as expected (see Table 1). Still, the shape model extent along the spin axis is poorly constrained, probably due to the lack of data from geometries where intermediate amplitudes could be observed.

\section{TPM analysis}

We denote the two mirror solutions as AM 1 and AM 2. We used 49 thermal observations, 24 from IRAS $(8 \times 12 \mu \mathrm{m}, 8 \times 25 \mu \mathrm{m}$, $8 \times 60 \mu \mathrm{m})$, eight from AKARI $(4 \times \mathrm{S} 9 \mathrm{~W}, 4 \times \mathrm{L} 18 \mathrm{~W})$, and 17 from WISE $(8 \times \mathrm{W} 3,9 \times \mathrm{W} 4)$. The Bond albedo was assumed to be $A=0.070$.

Model AM 1 is significantly better than AM 2 and the spheres in this case, although the reduced $\chi^{2}$ is 1.1 , slightly over unity (Table B.9, and the $\chi^{2}$ plot in Fig. B.18). The IRAS data residuals show some scatter and the WISE data are reasonably well fitted but still present some waviness in the OMR versus rotational phase plot (Figs. 9 and B.18). The latter could indicate that there is room for improvement of the shape model. Very high 

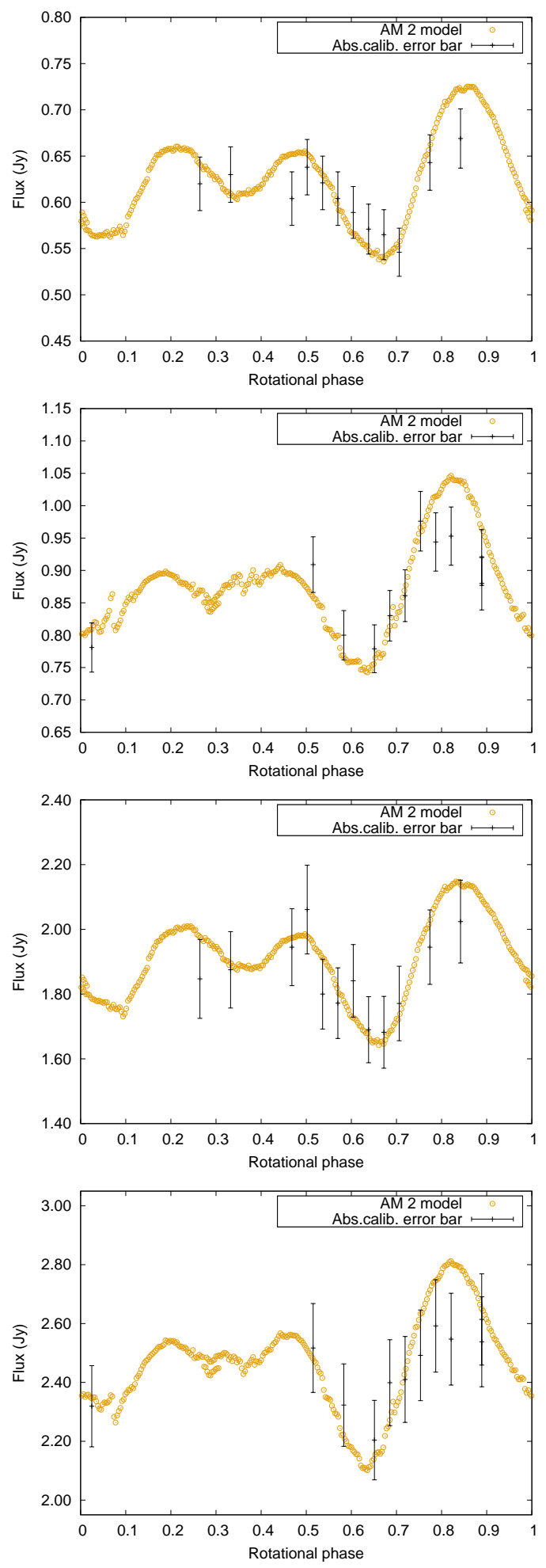

Fig. 8. Asteroid (538) Friederike's WISE W3 (first two plots from the top) and W4 data (last two plots) taken at two epochs, and thermal lightcurves for shape model AM 2 and the corresponding best-fitting solutions obtained based on all thermal data.

roughness solutions fit the data better, and an rms lower than 0.3 can be rejected at the $3 \sigma$ level.

We conclude that additional densely sampled thermal lightcurves and an improved shape model could improve the constraints on the thermal inertia and surface roughness. With a diameter $46_{-2}^{+4} \mathrm{~km}$, we get $p_{\mathrm{V}}=0.18_{-0.03}^{+0.02}$, and the best fit is obtained for medium values of thermal inertia.
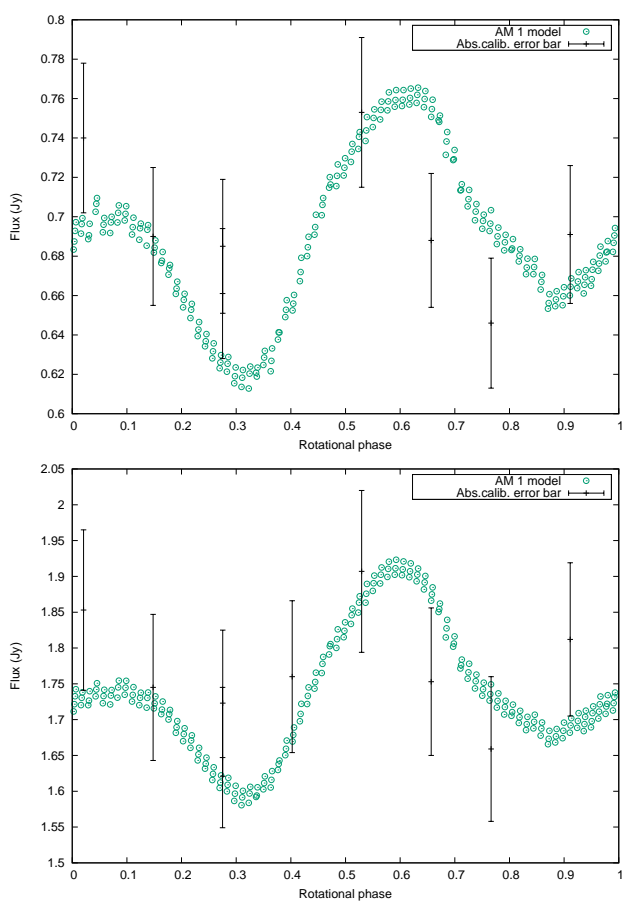

Fig. 9. Asteroid (653) Berenike WISE data (W3 top, W4 bottom) and the AM 1 model's thermal lightcurves (see Table B.9).

\subsection{0. (673) Edda}

The first published lightcurve of Edda was obtained within our project (Marciniak et al. 2016), and was highly asymmetric, with maxima unequally spaced in time. Here we present data from five more apparitions, confirming the period of $22.34 \mathrm{~h}$, and nontypical, asymmetric lightcurve behaviour (see Figs. A.43-A.47). This dataset was complemented by one more apparition with partial coverage by data from the SuperWASP archive (Grice et al. 2017). lightcurve amplitudes ranged from 0.13 to 0.23 mag. The shape model from lightcurve inversion is somewhat angular, and the fit to some of the lightcurves of smallest amplitude is imperfect. Both spin solutions are well constrained and are given in Table 1.

\section{TPM analysis}

The two mirror solutions are named AM 1 and AM 2. At our disposal there were 54 infrared observations, 20 from IRAS $(7 \times 12 \mu \mathrm{m}, 7 \times 25 \mu \mathrm{m}, 6 \times 60 \mu \mathrm{m})$, six from AKARI $(3 \times \mathrm{S} 9 \mathrm{~W}$ and $3 \times \mathrm{L} 18 \mathrm{~W})$, and $28 \mathrm{WISE}(14 \times \mathrm{W} 3$ and $14 \times \mathrm{W} 4)$. We assumed $A=0.047$ for the Bond albedo.

The best solutions for both models have comparable and very low $\bar{\chi}_{m}^{2}$ with very low thermal inertia of about 3 SI units (see Table B.10 and the $\chi^{2}$ plots in Fig. B.19). The low $\bar{\chi}_{\mathrm{m}}^{2} \mathrm{~s}$ suggest the error bars might be overestimated, so we normalise the $\bar{\chi}^{2}$ curves to have the minimum at 1 in order to compute the uncertainties of the parameters (see discussion in Hanuš et al. 2015). The shapes fit the WISE data well (Figs. 10 and B.7), and unlike (195) Eurykleia's case, the best-fitting solution also fits the AKARI data with a reasonably flat OMR versus wavelength plot (Fig. B.19).

To conclude, although high thermal inertias of about 70 SI units are still allowed, the best solutions seem to point to very low thermal inertias of around 3 SI units. The diameters are constrained to be $38_{-2}^{+6} \mathrm{~km}$ ( $3 \sigma$ error bars), which lead to a visible geometric albedo of $p_{\mathrm{V}}=0.13_{-0.05}^{+0.03}$. This value is 

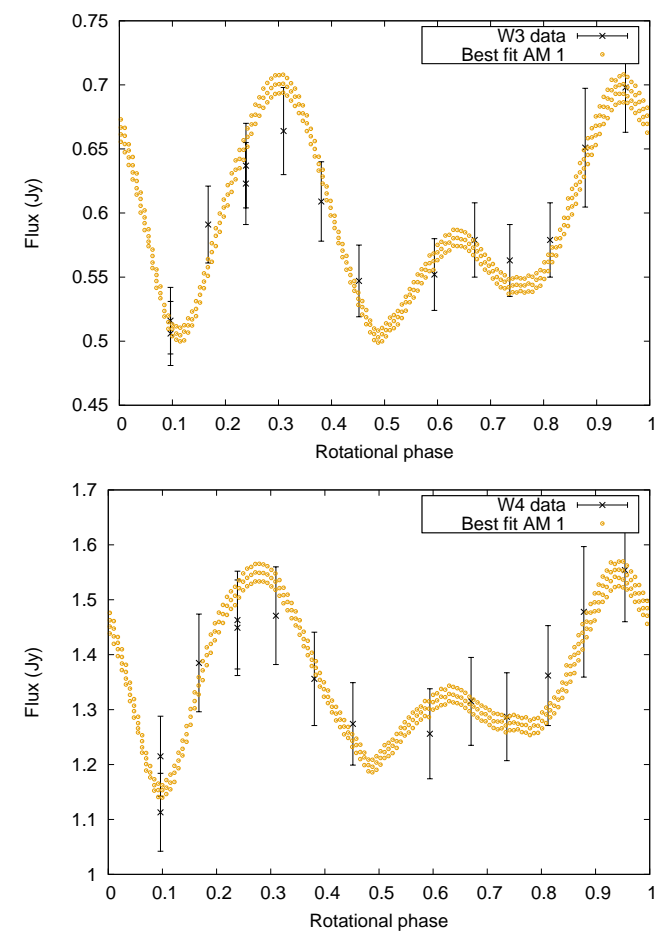

Fig. 10. Asteroid (673) Edda's WISE data and model thermal lightcurves for shape model 1's best-fitting solution (very low thermal inertia of 3 SI units).

somewhat low for a typical S-type (classified based on a visible SMASS spectrum, Small Main-Belt Asteroid Spectroscopic Survey Bus \& Binzel 2002a,b).

\subsection{1. (834) Burnhamia}

Previously observed by Buchheim (2007) and in our campaign, Burnhamia displayed lightcurves of extrema at unequal levels, $13.87 \mathrm{~h}$ period, and amplitudes from 0.15 to 0.25 mag. We accumulated data from six apparitions well spread in longitude, and present them in Figs. A.48-A.53. In the lightcurve inversion we obtained two well-defined pole solutions (Table 1) with somewhat unconstrained shape models both in the vertical dimension and in the level of smoothness of the shape.

\section{TPM analysis}

The two mirror solutions are denoted AM 1 and AM 2. We used 70 thermal observations, 42 from IRAS $(11 \times 12 \mu \mathrm{m}, 11 \times 25 \mu \mathrm{m}$, $11 \times 60 \mu \mathrm{m}, 9 \times 100 \mu \mathrm{m})$, one from AKARI (L18W), and 26 from WISE $(13 \times \mathrm{W} 3,13 \times \mathrm{W} 4)$. We assumed $A=0.035$ for the Bond albedo.

It turned out to be yet another slow rotator with seemingly low thermal inertia. The three-sigma upper limit on $50 \mathrm{SI}$ is quite clear. The $\bar{\chi}_{\min }^{2}$ is $\sim 0.8$ for both models (Table B.11, and the $\chi^{2}$ plots in Fig. B.20), so the fit is good but there is room for improvement. The WISE data are reasonably well fitted (Fig.11, and B.8), but the $100 \mu \mathrm{m}$ IRAS data are not (these data carry less weigh for the fit, however).

As usual, the roughness is not constrained, the diameter is $67_{-6}^{+8} \mathrm{~km}$ and $p_{\mathrm{V}}=0.074_{-0.016}^{+0.014}$. We could benefit from additional thermal light curves at positive phase angles (pre-opposition) and lower heliocentric distance although there is no apparent trend in the lowest panel of Fig. B.20.
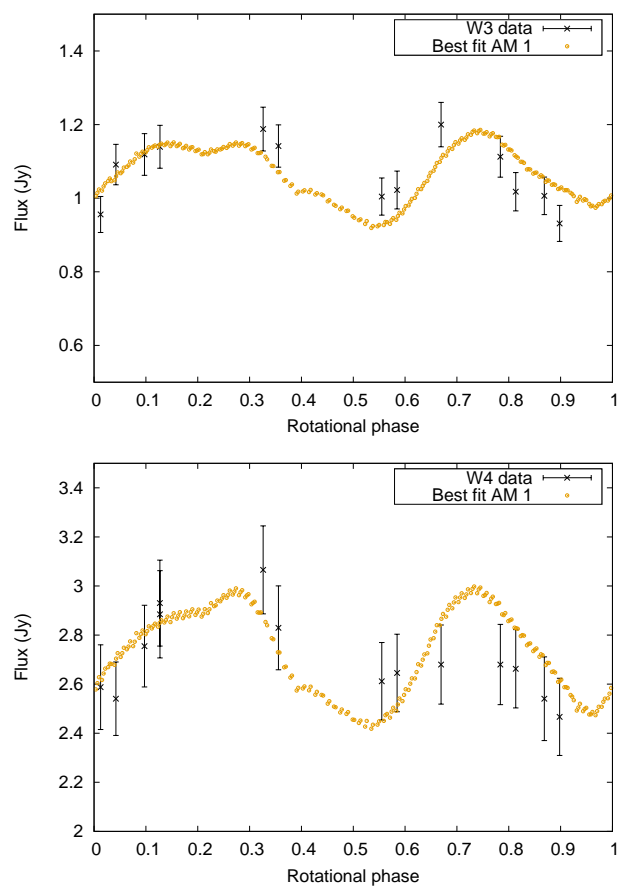

Fig. 11. Asteroid (834) Burnhamia's WISE data and model thermal lightcurves for shape model 1's best-fitting solution (see Table B.11).

\section{Discussion and conclusions}

We present here 11 new spin and shape models of slowly rotating asteroids from lightcurve inversion on dense lightcurves, with determined sizes, albedos, and thermal parameters. Together with five models from our previous study (Marciniak et al. 2018), we have 16 shape models within our sample of slow-rotators with applied TPM. This number substantially enlarges the available pool of well-studied slow rotators. Models obtained in this work provide good resolution, "smooth" shape representations for a multitude of future applications, including calibration in the infrared.

One of our aims was to verify whether the thermal inertia indeed increases with the rotation period (Harris \& Drube 2016). Our initial sample (Marciniak et al. 2018) seemed to be in line with this finding, while our current sample is dominated by targets with lower thermal inertias. To ensure this different result is not related to the fact that we used a different code for this work ${ }^{5}$, we modelled one of the targets from Marciniak et al. (2018) and reproduced the results. This is expected given that we use the same model approach and approximations. We also cross-checked our results with those estimated by Harris \& Drube (2016) and found one overlapping target, 487 Venetia, with consistent thermal inertia values: 96 SIu in Harris \& Drube (2016), and $100 \pm 75$ SIu in Marciniak et al. (2018). In Fig. 12 we superimposed our results on the plot from Harris \& Drube (2016).

Taking both of our samples together (from this work and from Marciniak et al. 2018), we find diverse values of thermal inertia, ranging from 3 up to 125 SI units. To make a quantitative comparison, we collected the thermal inertias of all targets with sizes $30<D<200 \mathrm{~km}$ from Hanuš et al. (2018a), Delbo' et al. (2015), and our values and split them into two groups: slow $(P>12 \mathrm{~h})$ and fast $(P<12 \mathrm{~h})$ rotators. Neither the two-sample

\footnotetext{
5 We used the TPM code of Müller (2002) in Marciniak et al. (2018), whereas here we used the TPM code of Delbo' \& Harris (2002) modified in Alí-Lagoa et al. (2014) mentioned in Sect. 3.
} 


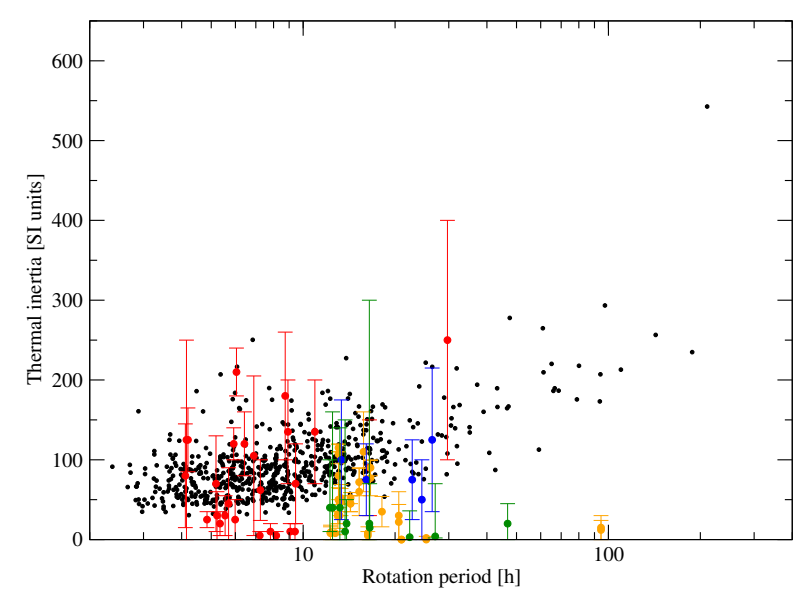

Fig. 12. Updated plot from Harris \& Drube (2016) of thermal inertia dependence on rotation period. Black dots are thermal inertias estimated from $\eta$ values by Harris \& Drube (2016), red dots from detailed TPM compiled by Delbo' et al. (2015), orange are slow rotators from "VariedShape TPM" by Hanuš et al. (2018a), blue are from TPM results of Marciniak et al. (2018), and green dots from this work. Our new results do not confirm the growing trend.

Kolmogorov-Smirnov test nor the k-sample Anderson-Darling test rule out the null hypothesis that both samples (with 33 and 36 values, respectively) are drawn from the same distribution. The $p$-values are higher than 0.3 , far even from a lax threshold of statistical significance of 5\%. Perhaps a larger sample could lead to a firmer conclusion in the future.

This does not necessarily deny the hypothesis of $\Gamma$ growth with period; instead it might indicate various levels of regolith development on the surface, connected to age and/or collisional history, and space weathering. This could be further tested if we had sufficiently large samples of objects belonging to young and old collisional families, since younger surfaces are expected to have less developed regoliths (e.g. Delbo' et al. 2014). The interpretation of our results might also be complicated by the temperature dependence of the thermal conductivity, which we account for by assuming the scaling relation between $\Gamma$ and heliocentric distance given for example in Eq. (13) of Delbo' et al. (2015) to normalise the values of $\Gamma$ to 1 au (these values are given in the last column of Table 2). This scaling is related to the $T^{3}$ dependence of the thermal conductivity (e.g. Vasavada et al. 1999), and translates into a thermal inertia dependence of $\Gamma \propto T^{3 / 2}$. However, we note that Centaurs and TNOs (trans-Neptunian objects) follow a different behaviour ( $\Gamma \propto T^{2}$; Lellouch et al. 2013), and Rozitis \& et al. (2018) have found more extreme dependencies in two near-Earth asteroids $\left(\Gamma \propto T^{4.4}\right.$ and $\Gamma \propto T^{2.92}$ ), although the fitted exponents have large error bars. More work is certainly needed in this direction.

Our large observing campaign, targeted at about 100 asteroids with slow rotation and small amplitudes, already resulted in the gathering of around $10000 \mathrm{~h}$ of photometric data, where hundreds of hours are needed for a unique lightcurve inversion solution for each of such targets. There are a few factors further limiting the number of targets with all the parameters uniquely determined, like shape model imperfections, or thermal data limited geometries. In the future we are planning to add more photometric data to our datasets accumulated already including photometry from the TESS (Transiting Exoplanet Survey Satellite) mission, which should improve the uniqueness of many spin and shape solutions for targets not presented here. The TESS mission is observing in a similar cadence to the Kepler Space Telescope. Moreover, unlike in the case of Kepler, full frames are going to be downlinked, so TESS data are going to be perfect for extensive studies of slow rotators (Pál et al. 2018). It is also possible that the shape models that best fit visual lightcurves were not the best possible ones from the thermal point of view, so the varied-shape TPM method (Hanuš et al. 2015), or simultaneous fitting of visual and thermal data using a convex inversion TPM (Ďrech et al. 2017) could help to resolve issues with unconstrained solutions. To overcome problems with limited thermal data we are planning proposals to VLT/VISIR (Very Large Telescope with the VLT spectrometer and imager for the mid-infrared) and SOFIA (Stratospheric Observatory For Infrared Astronomy) infrared facilities, carefully choosing targets and observing geometries to best complement the existing datasets in terms of aspect, pre- and post-opposition geometry, and heliocentric distance.

Acknowledgements. This work was supported by the National Science Centre, Poland, through grant no. 2014/13/D/ST9/01818. The research leading to these results has received funding from the European Union's Horizon 2020 Research and Innovation Programme, under Grant Agreement no 687378 (SBNAF). The research of V.K. was supported by a grant from the Slovak Research and Development Agency, number APVV-15-0458. R.D. acknowledges financial support from the State Agency for Research of the Spanish MCIU through the "Center of Excellence Severo Ochoa" award for the Instituto de Astrofísica de Andalucía(SEV-2017-0709). The Joan Oró Telescope (TJO) of the Montsec Astronomical Observatory (OAdM) is owned by the Catalan Government and operated by the Institute for Space Studies of Catalonia (IEEC). This article is based on observations made in the Observatorios de Canarias del IAC with the $0.82 \mathrm{~m}$ IAC 80 telescope operated on the island of Tenerife by the Instituto de Astrofísica de Canarias (IAC) in the Observatorio del Teide. This article is based on observations made with the SARA telescopes (Southeastern Association for Research in Astronomy), whose nodes are located at the Observatorios de Canarias del IAC on the island of La Palma in the Observatorio del Roque de los Muchachos; Kitt Peak, AZ under the auspices of the National Optical Astronomy Observatory (NOAO); and Cerro Tololo Inter-American Observatory (CTIO) in La Serena, Chile. This project uses data from the SuperWASP archive. The WASP project is currently funded and operated by Warwick University and Keele University, and was originally set up by Queen's University Belfast, the Universities of Keele, St. Andrews, and Leicester, the Open University, the Isaac Newton Group, the Instituto de Astrofisica de Canarias, the South African Astronomical Observatory, and by STFC. Funding for the Kepler and K2 missions is provided by the NASA Science Mission Directorate. The data presented in this paper were obtained from the Mikulski Archive for Space Telescopes (MAST). STScI is operated by the Association of Universities for Research in Astronomy, Inc., under NASA contract NAS5- 26555. Support for MAST for non-HST data is provided by the NASA Office of Space Science via grant NNX09AF08G and by other grants and contracts. This publication makes use of data products from the Wide-field Infrared Survey Explorer, which is a joint project of the University of California, Los Angeles, and the Jet Propulsion Laboratory/California Institute of Technology, funded by the National Aeronautics and Space Administration. The research leading to these results has received funding from the LP2012-31 and LP2018-7/2018 Lenduilet grants of the Hungarian Academy of Sciences.

\section{References}

Alí-Lagoa, V., Lionni, L., Delbo, M., et al. 2014, A\&A, 561, A45 Alí-Lagoa, V., Müller, T. G., Usui, F., \& Hasegawa, S. 2018, A\&A, 612, A85 Binzel, R. P. 1987, Icarus, 72, 135

Blanco, C., Cigna, M., \& Riccioli, D. 2000, Planet. Space Sci., 48, 973 Bottke, W. F., Durda, D. D., Nesvorný, D., et al. 2005, Icarus, 175, 111

Buchheim, R. K. 2007, Minor Planet Bull., 34, 68

Bus, S. J., \& Binzel, R. P. 2002a, Icarus, 158, 146

Bus, S. J., \& Binzel, R. P. 2002b, Icarus, 158, 106

Carry, B. 2012, Planet. Space Sci., 73, 98

Cibulková, H., Ďurech, J., Vokrouhlický, D., Kaasalainen, M., \& Oszkiewicz,

D. A. 2016, A\&A, 596, A57

Cutri, R. M., et al. 2012, VizieR Online Data Catalog: II/311

Delbo', M., \& Harris, A. W. 2002, Meteorit. Planet. Sci., 37, 1929

Delbo', M., \& Tanga, P. 2009, Planet. Space Sci., 57, 259

Delbo', M., Libourel, G., Wilkerson, J., et al. 2014, Nature, 508, 233

Delbo', M., Mueller, M., Emery, J. P., Rozitis, B., \& Capria, M. T. 2015,

Asteroids IV, eds. P. Michel, F. E. DeMeo, \& W. F. Bottke (Tucson, AZ: University of Arizona Press), 107

Dunham, D. W., Herald, D., Frappa, E., et al. 2016, NASA Planetary Data System, 243, EAR 
Ďurech, J., \& Hanuš, J. 2018, A\&A, 620, A91

Durech, J., Sidorin, V., \& Kaasalainen, M. 2010, A\&A, 513, A46

Durech, J., Carry, B., Delbo, M., Kaasalainen, M., \& Viikinkoski, M. 2015, Asteroids IV, eds. P. Michel, F. E. DeMeo, \& W. F. Bottke (Tucson, AZ: University of Arizona Press), 183

Ďurech, J., Hanuš, J., Oszkiewicz, D., \& Vančo, R. 2016, A\&A, 587, A48

Ďurech, J., Delbo', M., Carry, B., Hanuš, J., \& Alí-Lagoa, V. 2017, A\&A, 604, A27

Ďurech, J., Hanuš, J., \& Alí-Lagoa, V. 2018, A\&A, 617, A57

Galad, A., \& Kornos, L. 2008, Minor Planet Bull., 35, 144

Galad, A., Vilagi, J., Kornos, L., \& Gajdos, S. 2009, Minor Planet Bull., 36, 116

Gil-Hutton, R. 1990, Rev. Mex. Astron. Astrofis., 21, 593

Grice, J., Snodgrass C., Green S., Parley N., \& Carry, B. 2017, Asteroids, Comets, meteors: ACM 2017

Gundlach, B., \& Blum, J. 2013, Icarus, 223, 479

Hainaut-Rouelle, M.-C., Hainaut, O. R., \& Detal, A. 1995, A\&AS, 112, 125

Hanuš, J., Ďurech, J., Brož, M., et al. 2011, A\&A, 530, A134

Hanuš, J., Ďurech, J., Brož, M., et al. 2013, A\&A, 551, A67

Hanuš, J., Delbo', M., Ďrech, J., \& Alí-Lagoa, V. 2015, Icarus, 256, 101

Hanuš, J., Ďurech, J., Oszkiewicz, D. A., et al. 2016, A\&A, 586, A108

Hanuš, J., Delbo', M., Ďurech, J., \& Alí-Lagoa, V. 2018a, Icarus, 309, 297

Hanuš, J., Delbo', M., Alí-Lagoa, V., et al. 2018b, Icarus, 299, 84

Harris, A. W., \& Drube, L. 2016, ApJ, 832, 127

Harris, A. W., \& Young, J. W. 1989, Icarus, 81, 314

Harris, A. W., Young, J. W., Dockweiler, T., et al. 1992, Icarus, 95, 115

Howell, S. B., Sobeck, C., Haas, M., et al. 2014, PASP, 126, 398

Kaasalainen, M., \& Torppa, J. 2001, Icarus, 153, 24

Kaasalainen, M., Torppa, J., \& Muinonen, K. 2001, Icarus, 153, 37

Kryszczyńska, A. 2013, A\&A, 551, A102

Lagerkvist, C.-I., Erikson, A., Debehogne, H., et al. 1995, A\&AS, 113, 115

Lagerros, J. S. V. 1996, A\&A, 310, 1011

Lagerros, J. S. V. 1998, A\&A, 332, 1123

Lellouch, E., Santos-Sanz, P., Lacerda, P., et al. 2013, A\&A, 557, A60

Licandro, J., Hargrove, K., Kelley, M., et al. 2012, A\&A, 537, A73

Lim, L. F., Emery, J. P., \& Moskovitz, N. A. 2011, Icarus, 213, 510

Mainzer, A., Grav, T., Masiero, J., et al. 2011, ApJ, 741, 90

Mainzer, A. K., Bauer, J. M., Cutri, R. M., et al. 2016, NASA Planetary Data System, 247

Marciniak, A., Pilcher, F., Oszkiewicz, D., et al. 2015, Planet. Space Sci., 118, 256

Marciniak, A., Pilcher, F., Oszkiewicz, D., et al. 2016, in 37th Meeting of the Polish Astronomical Society, eds. A. Różańska \& M. Bejger (Warsaw: Polish Astronomical Society), 3, 84

Marciniak, A., Bartczak, P., Müller, T., et al. 2018, A\&A, 610, A7

Marzari, F., Rossi, A., \& Scheeres, D. J. 2011, Icarus, 214, 622

Masiero, J. R., Mainzer, A. K., Grav, T., et al. 2011, ApJ, 741, 68

Matter, A., Delbo, M., Carry, B., \& Ligori, S. 2013, Icarus, 226, 419

Molnár, L., Pál, A., Sárneczky, K., et al. 2018, ApJS, 234, 37

Müller, T. G. 2002, Meteorit. Planet. Sci., 37, 1919

Müller, T. G., \& Lagerros, J. S. V. 1998, A\&A, 338, 340

Müller, T. G., \& Lagerros, J. S. V. 2002, A\&A, 381, 324

Oszkiewicz, D. A., Muinonen, K., Bowell, E., et al. 2011, J. Quant. Spectr. Rad. Transf., 112, 1919

Pál, A. 2012, MNRAS, 421, 1825

Pál, A., Szabó, R., Szabó, G. M., et al. 2015, ApJ, 804, L45

Pál, A., Molnár, L., \& Kiss, C. 2018, PASP, 130, 114503

Pilcher, F. 2013, Minor Planet Bull., 40, 85

Pilcher, F., \& Martinez, L. 2015, Minor Planet Bull., 42, 239

Press, W. H., Flannery, B. P., \& Teukolsky, S. A. 1986, Numerical Recipes. The Art of Scientific Computing (Cambridge: Cambridge University Press)

Rozitis, B., Green, S. F., MacLennan, E., \& Emery, J. P. 2018, Icarus, 477, 1782

Santana-Ros, T., Bartczak, P., Michałowski, T., Tanga, P., \& Cellino, A. 2015, MNRAS, 450, 333

Slivan, S. M. 2002, Nature, 419, 49

Slivan, S. M. 2012, Minor Planet Bull., 39, 204

Szabó, R., Pál, A., Sárneczky, K., et al. 2016, A\&A, 596, A40

Szabó, G. M., Pál, A., Kiss, C., et al. 2017, A\&A, 599, A44

Tedesco, E. F. 1979, Ph.D. Thesis, New Mexico State University, NM, USA

Tedesco, E. F., Cellino, A., \& Zappalá, V. 2005, AJ, 129, 2869

Tholen, D. J. 1989, in Asteroids II, eds. R. P. Binzel, T. Gehrels, \& M. S. Matthews (Tucson, AZ: University of Arizona Press), 1139

Usui, F., Kuroda, D., Müller, T. G., et al. 2011, PASJ, 63, 1117

Vasavada, A. R., Paige, D. A., \& Wood, S. E. 1999, Icarus, 141, 179

Vereš, P., Jedicke, R., Fitzsimmons, A., et al. 2015, Icarus, 261, 34

Vokrouhlický, D., Bottke, W. F., Chesley, S. R., Scheeres, D. J., \& Statler, T. S 2015, Asteroids IV, eds. P. Michel, F. E. DeMeo, \& W. F. Bottke (Tucson, AZ: University of Arizona Press), 509
Warner, B. D. 2004, Minor Planet Bull., 31, 67

Warner, B. D., \& Harris, A. W. 2011, Icarus, 216, 610

Warner, B. D., Roy, R., Dyvig, R., et al. 2007, Minor Planet Bull., 34, 99

Warner, B. D., Harris, A. W., \& Pravec, P. 2009, Icarus, 202, 134

Wright, E. L., Eisenhardt, P. R. M., Mainzer, A. K., et al. 2010, AJ, 140, 1868

Zappala, V., Scaltriti, F., \& di Martino M. 1983, Icarus, 56, 325

1 Astronomical Observatory Institute, Faculty of Physics, A. Mickiewicz University, Słoneczna 36, 60-286 Poznań, Poland e-mail: am@amu .edu.pl

2 Max-Planck-Institut für Extraterrestrische Physik, Giessenbachstrasse 1, 85748 Garching, Germany

3 Konkoly Observatory, Research Centre for Astronomy and Earth Sciences, Hungarian Academy of Sciences, 1121 Budapest, Konkoly Thege Miklós út 15-17, Hungary

4 MTA CSFK Lendület Near-Field Cosmology Research Group, Budapest, Hungary

5 Astronomy Department, Eötvös Loránd University, Pázmány P. s. 1/A, 1171 Budapest, Hungary

6 Institute of Physics, University of Szczecin, Wielkopolska 15, 70-453 Szczecin, Poland

7 The IEA, University of Reading, Philip Lyle Building, Whiteknights Campus, Reading, RG6 6BX, UK

8 Observatoire des Hauts Patys, 84410 Bédoin, France

9 Villefagnan Observatory, Villefagnan, France

10 Geneva Observatory, 1290 Sauverny, Switzerland

11 Les Engarouines Observatory, 84570 Mallemort-du-Comtat, France

12 Stazione Astronomica di Sozzago, 28060 Sozzago, Italy

13 Departamento de Sistema Solar, Instituto de Astrofísica de Andalucía (CSIC), Glorieta de la Astronomía s/n, 18008 Granada, Spain

14 Rose-Hulman Institute of Technology, CM 1715500 Wabash Ave., Terre Haute, IN 47803, USA

15 Observatoire du Bois de Bardon, 16110 Taponnat, France

16 Elgin Observatory, 1155 Hartford St; Elgin, OR USA

17 Instituto de Astrofísica de Canarias, C/ Vía Lactea, s/n, 38205 La Laguna, Tenerife, Spain

18 Gran Telescopio Canarias (GRANTECAN), Cuesta de San José s/n, 38712 Breña Baja, La Palma, Spain

19 Lincaheira Observatory, Instituto Politécnico de Tomar, 2300-313 Tomar, Portugal

20 School of Physical Sciences, The Open University, MK7 6AA, UK

21 Chungbuk National University, 1, Chungdae-ro, Seowon-gu, Cheongju-si, Chungcheongbuk-do, Republic of Korea

22 Korea Astronomy and Space Science Institute, 776 Daedeokdae-ro, Yuseong-gu, 305-348 Daejeon, Korea

23 Institute of Physics, Faculty of Natural Sciences, University of P. J. Šafárik, Park Angelinum 9, 04001 Košice, Slovakia

24 Laboratory of Space Researches, Uzhhorod National University, Daleka st. 2a, 88000, Uzhhorod, Ukraine

25 Observatorio de Albox, Almeria, Spain

26 Observatório Nacional Rua General José Cristino, 77, 20921-400 Bairro Imperial de São Cristóvão Rio de Janeiro, RJ, Brasil

27 Mt. Suhora Observatory, Pedagogical University, Podchorążych 2, 30-084 Cracow, Poland

28 Organ Mesa Observatory, 4438 Organ Mesa Loop, Las Cruces, New Mexico 88011, USA

29 Command Module Observatory, 121 W. Alameda Dr., Tempe, AZ 85282, USA

30 Observatoire de Blauvac, 293 chemin de St Guillaume, 84570 St-Estève, France

31 Lowell Observatory, 1400 West Mars Hill Road, Flagstaff, AZ 86001, USA

32 Department of Physics, University of Adiyaman, 02040 Adiyaman, Turkey

33 Bisei Spaceguard Center, Japan Spaceguard Association, 1716-3, Okura, Bisei, Ibara, Okayama 714-1411, Japan

34 Kepler Institute of Astronomy, University of Zielona Góra, Lubuska 2, 65-265 Zielona Góra, Poland 


\section{Appendix A: Visible photometry}

Details of the observing runs (Table A.1) and composite lightcurves of asteroids with spin and shape models are presented here (Figs. A.1-A.53).

Table A.1. Observation details.

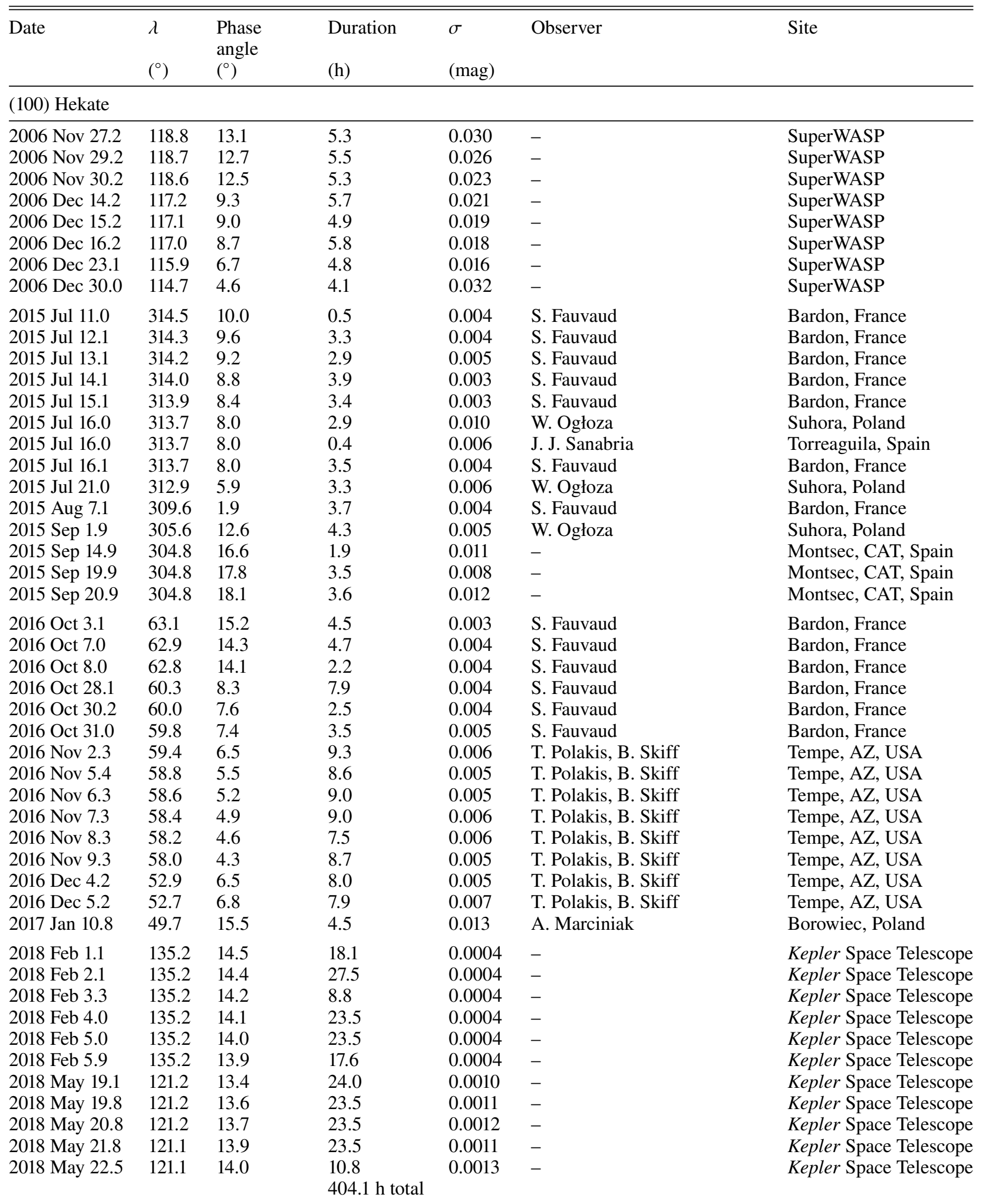

Notes. Columns are: mid-time observing date, ecliptic longitude of the target, sun-target-observer phase angle, duration of the observing run, photometric scatter, observer and site name. See Marciniak et al. (2018) for telescope and site details. The remaining part of the table for the rest of the targets is available at the CDS. 


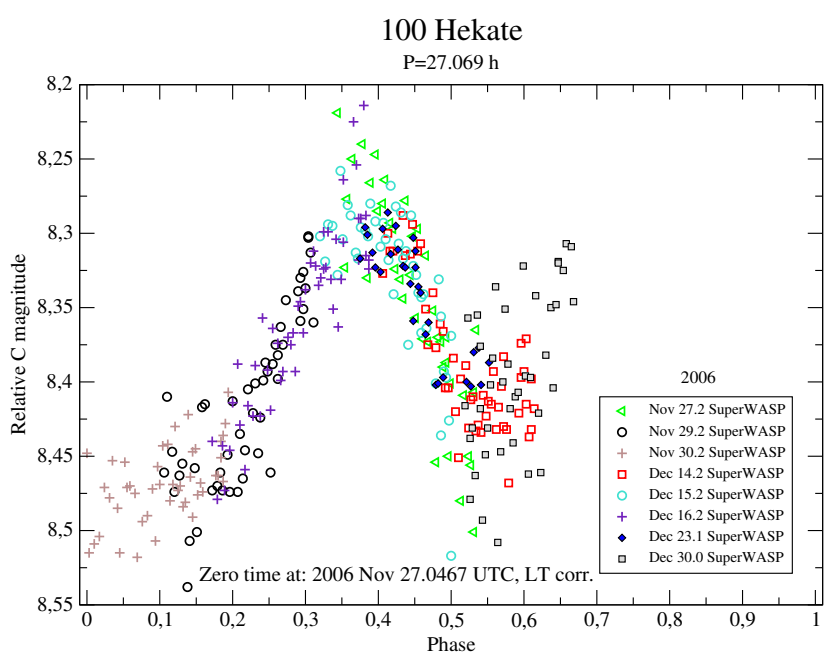

Fig. A.1. Composite lightcurve of (100) Hekate from the year 2006.

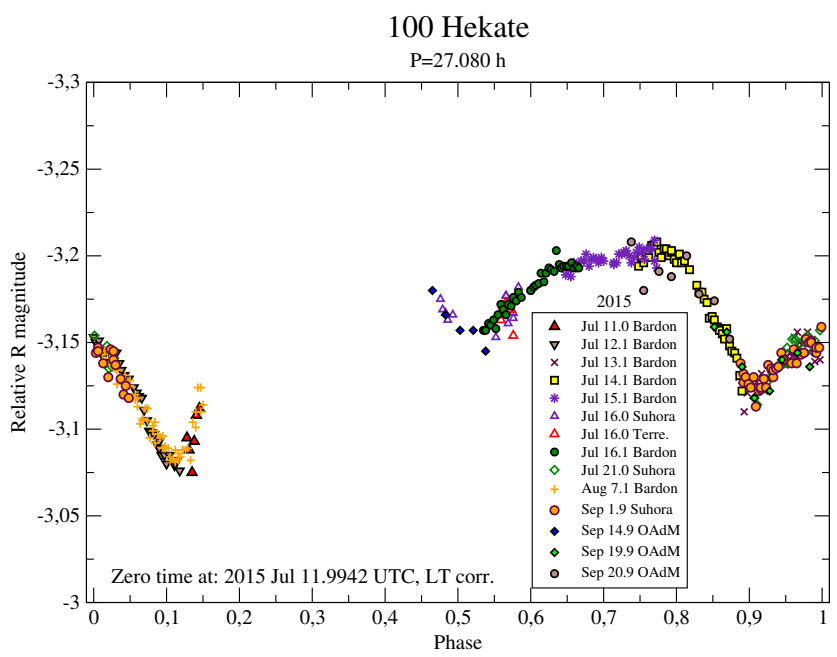

Fig. A.2. Composite lightcurve of (100) Hekate from the year 2015.

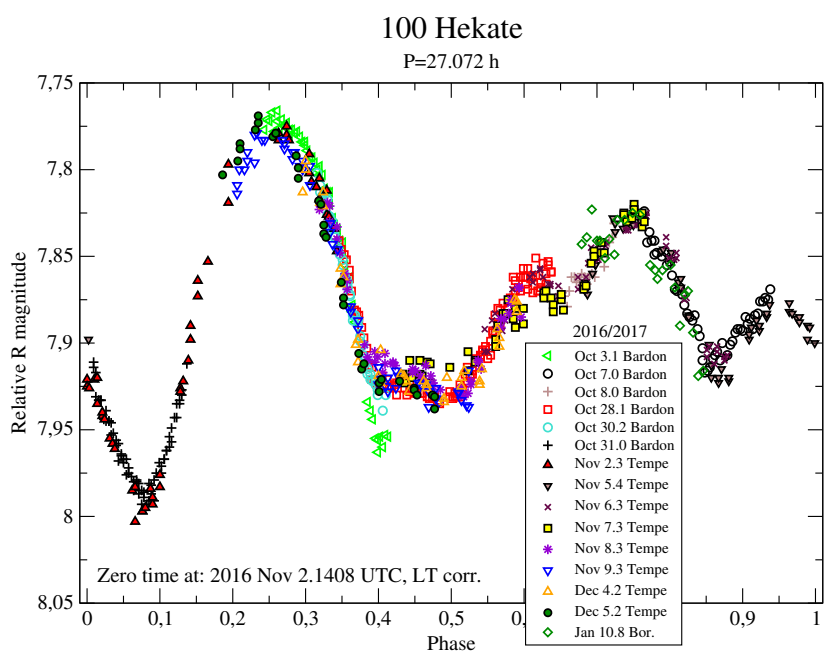

Fig. A.3. Composite lightcurve of (100) Hekate from the years 20162017.

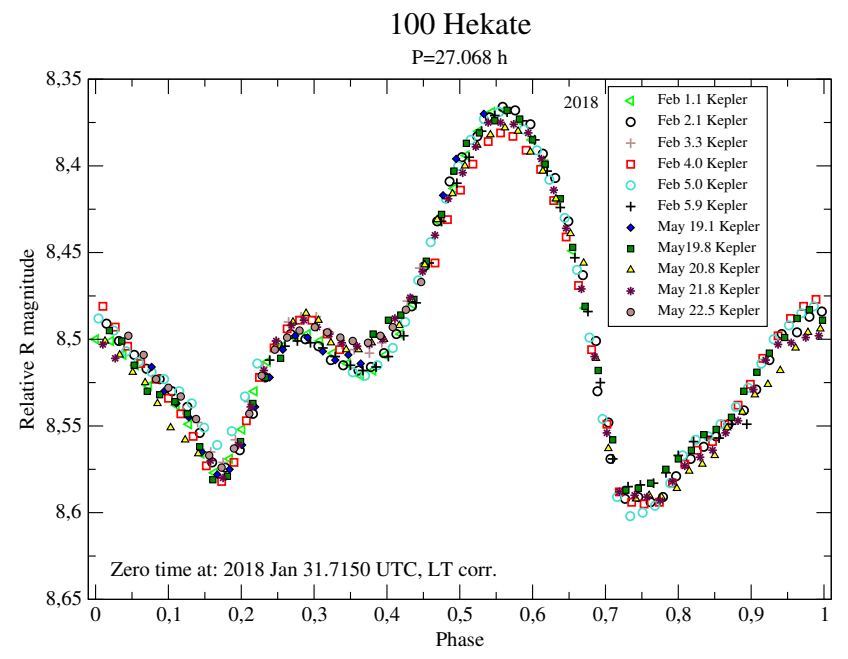

Fig. A.4. Composite lightcurve of (100) Hekate based on Kepler K2 data from the year 2018 .

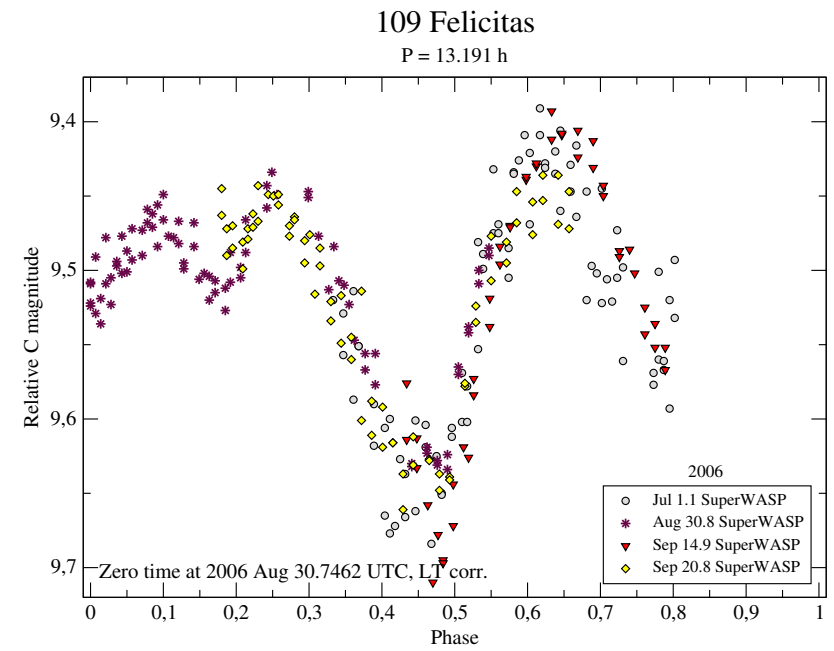

Fig. A.5. Composite lightcurve of (109) Felicitas from the year 2006.

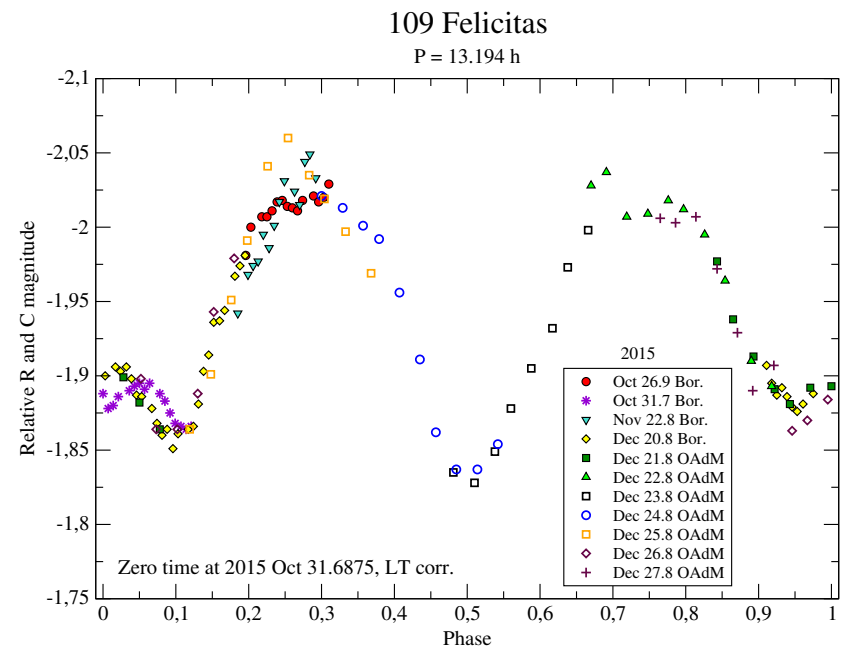

Fig. A.6. Composite lightcurve of (109) Felicitas from the year 2015. 


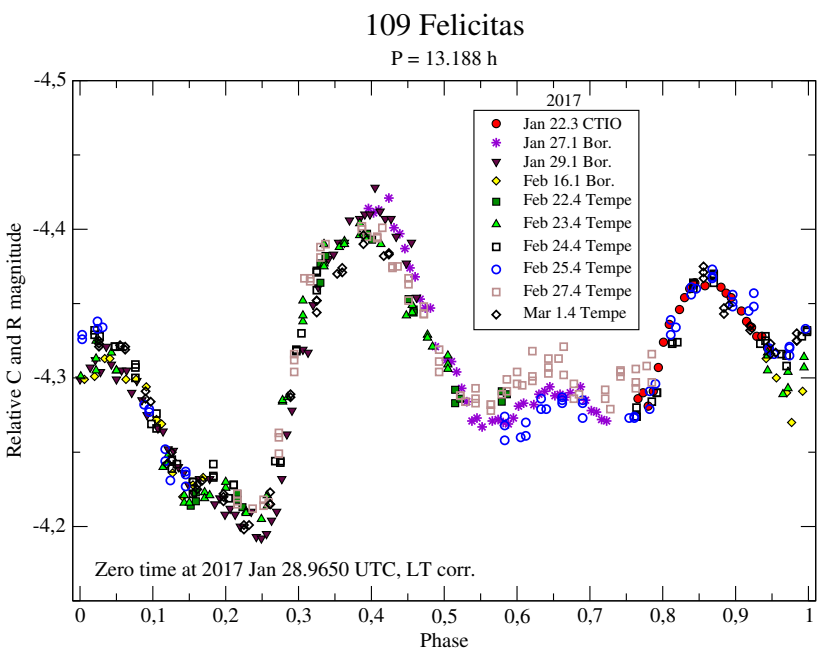

Fig. A.7. Composite lightcurve of (109) Felicitas from the year 2017.

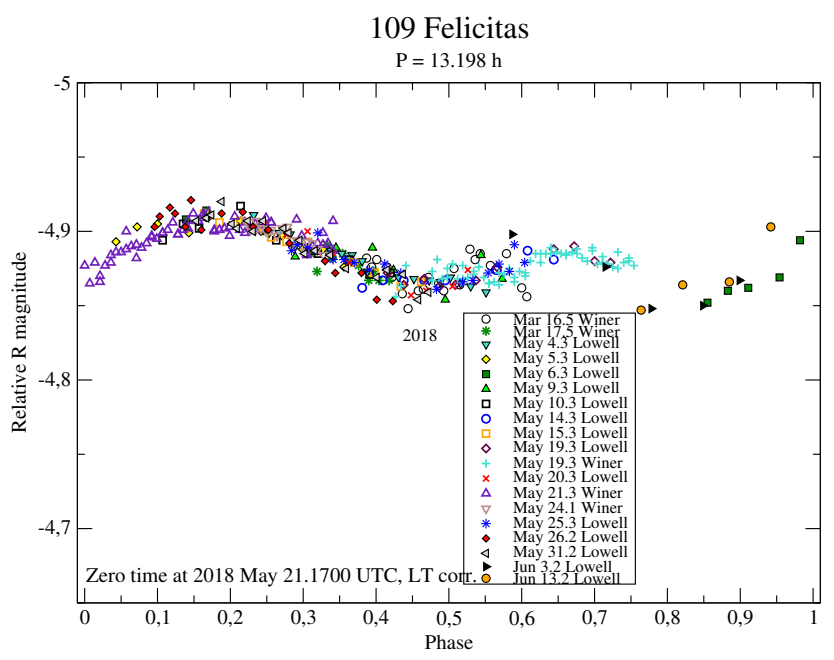

Fig. A.8. Composite lightcurve of (109) Felicitas from the year 2018.

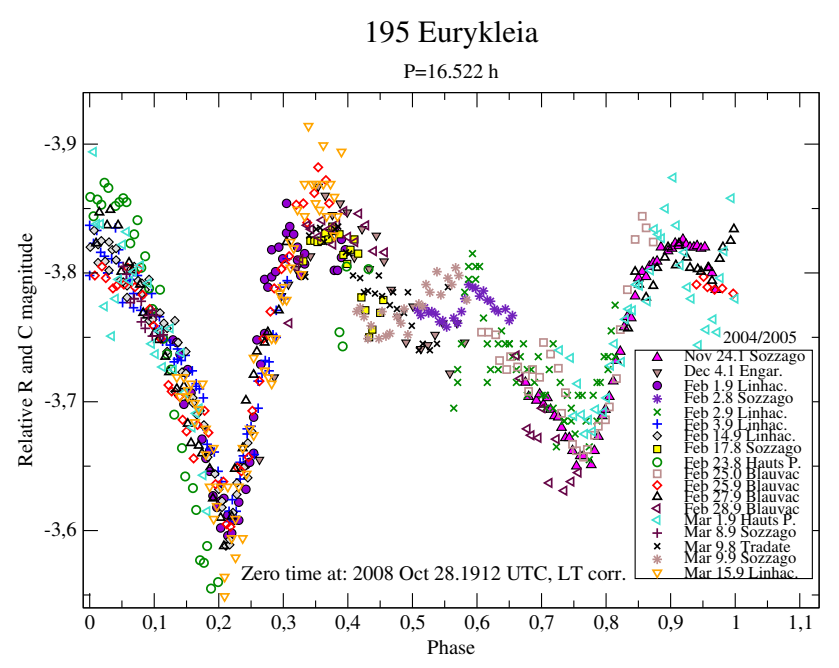

Fig. A.9. Composite lightcurve of (195) Eurykleia from the years 2004 2005.

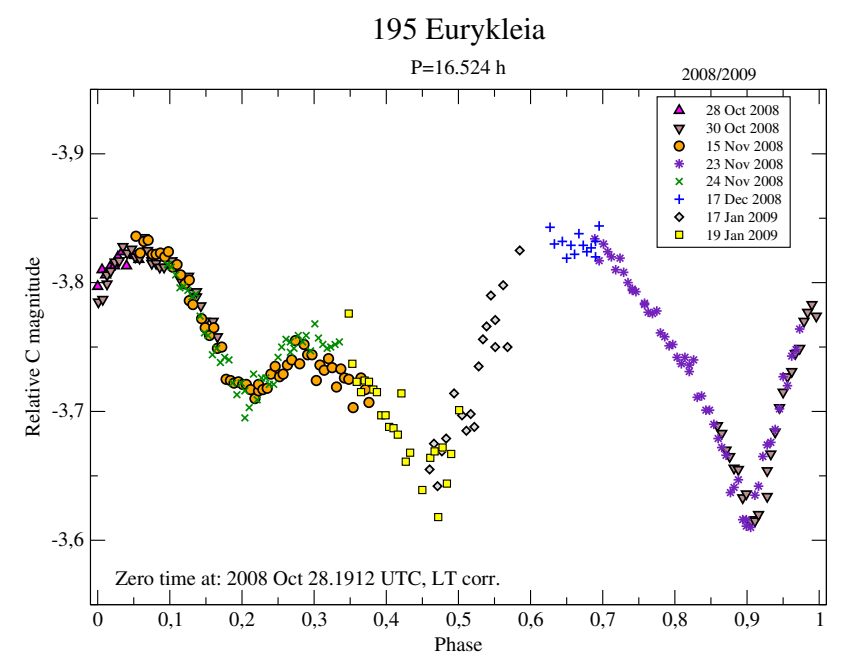

Fig. A.10. Composite lightcurve of (195) Eurykleia from the year 2008.

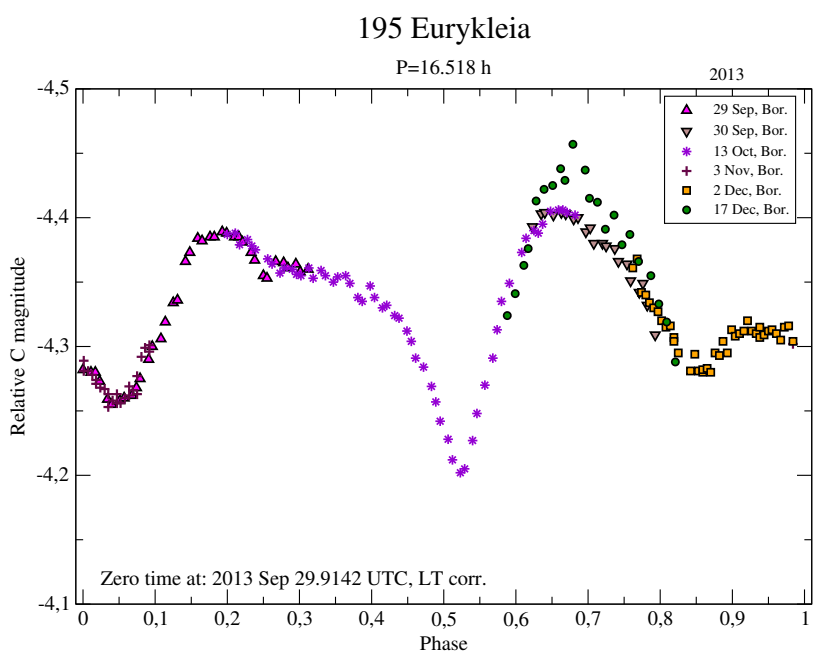

Fig. A.11. Composite lightcurve of (195) Eurykleia from the year 2013.

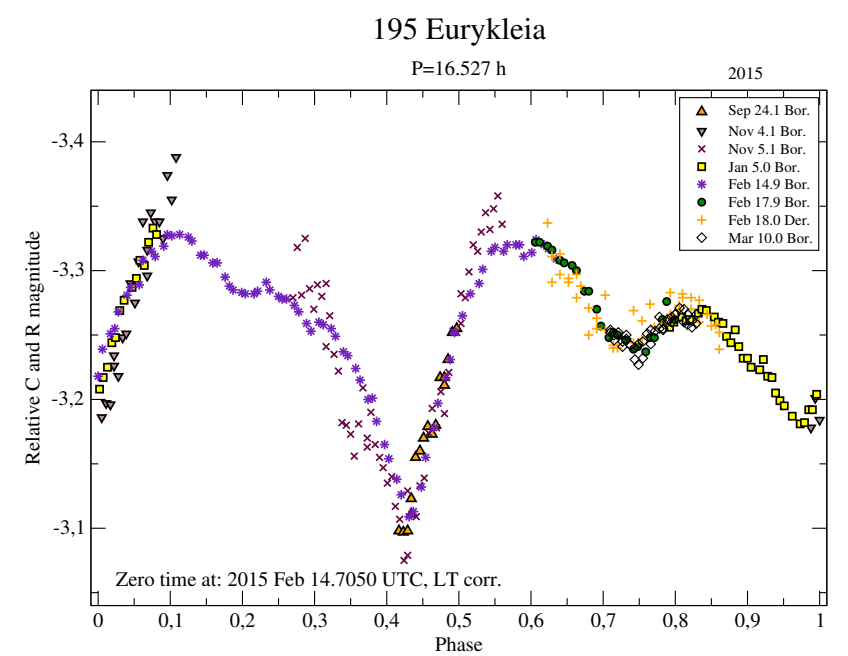

Fig. A.12. Composite lightcurve of (195) Eurykleia from the year 2015. 
195 Eurykleia

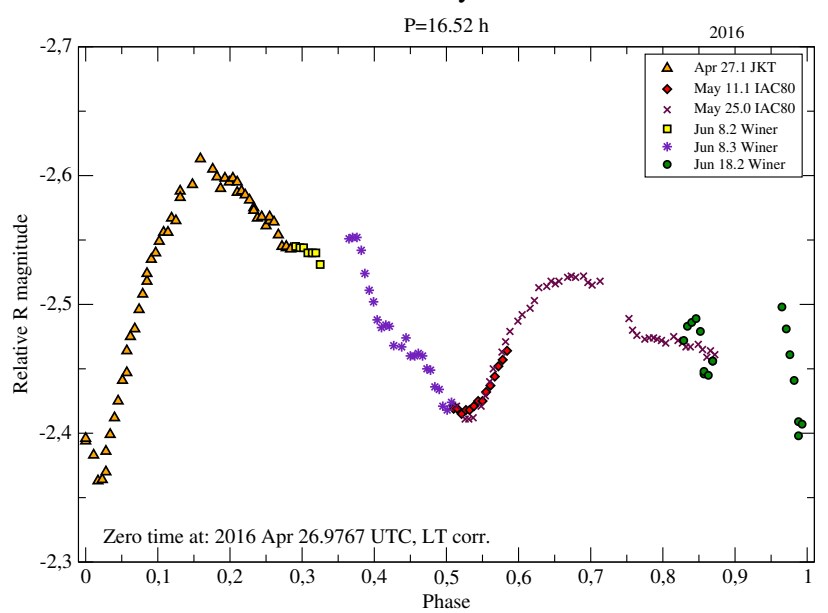

Fig. A.13. Composite lightcurve of (195) Eurykleia from the year 2016.

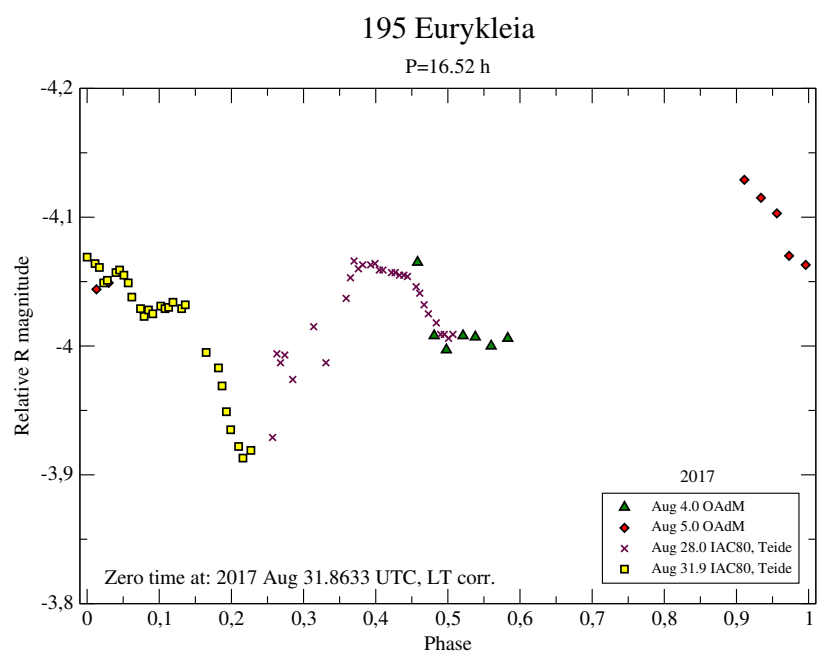

Fig. A.14. Composite lightcurve of (195) Eurykleia from the year 2017.

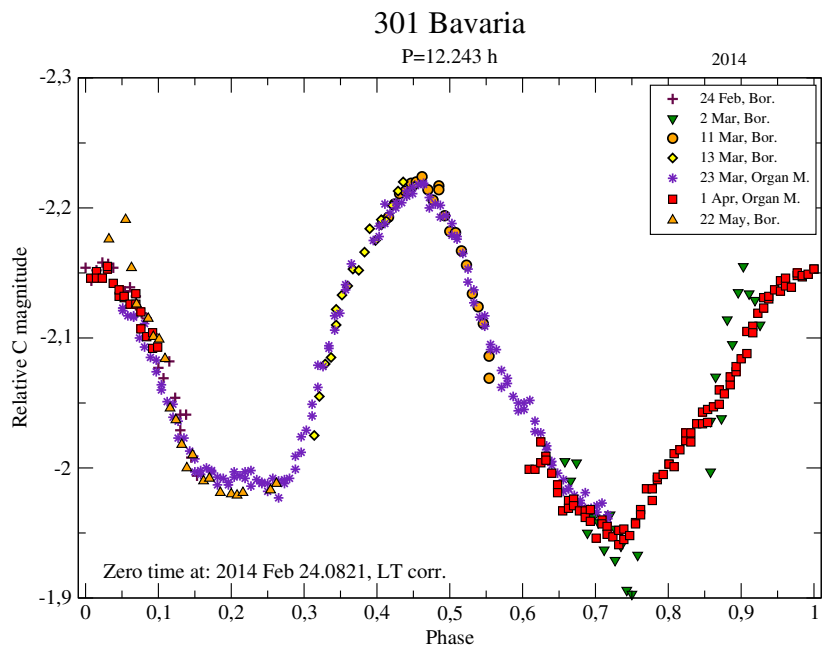

Fig. A.15. Composite lightcurve of (301) Bavaria from the year 2014.

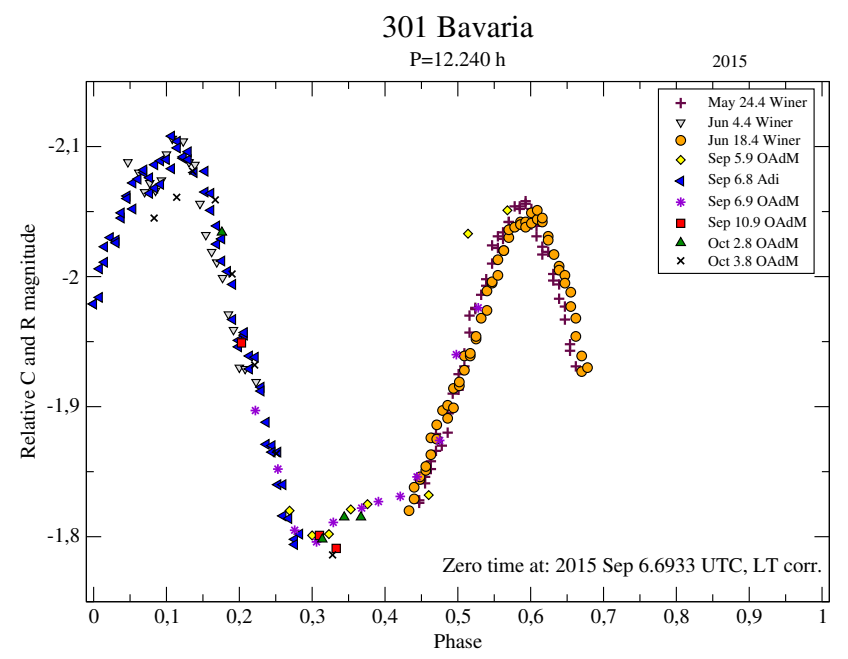

Fig. A.16. Composite lightcurve of (301) Bavaria from the year 2015.

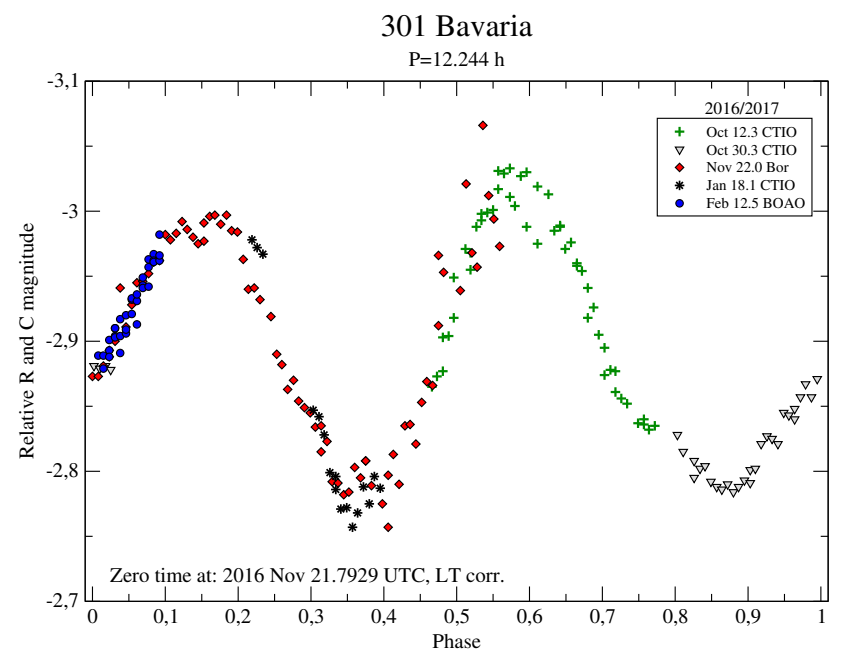

Fig. A.17. Composite lightcurve of (301) Bavaria from the years 20162017.

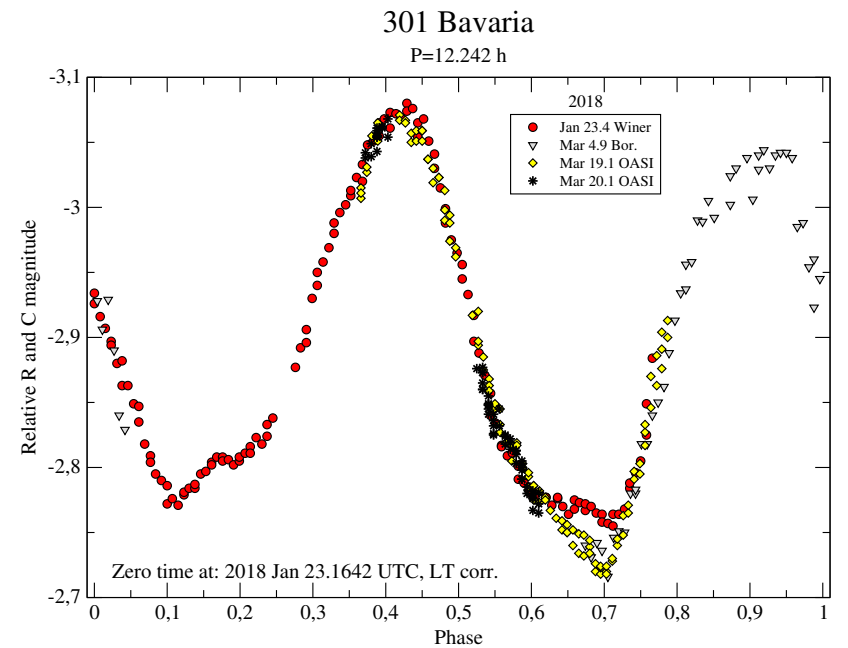

Fig. A.18. Composite lightcurve of (301) Bavaria from the year 2018. 


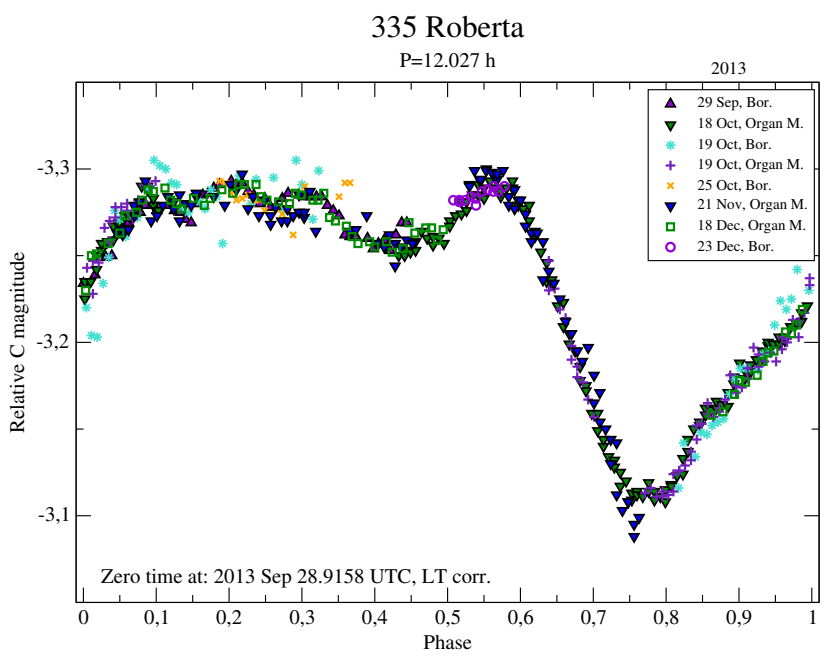

Fig. A.19. Composite lightcurve of (335) Roberta from the year 2013.

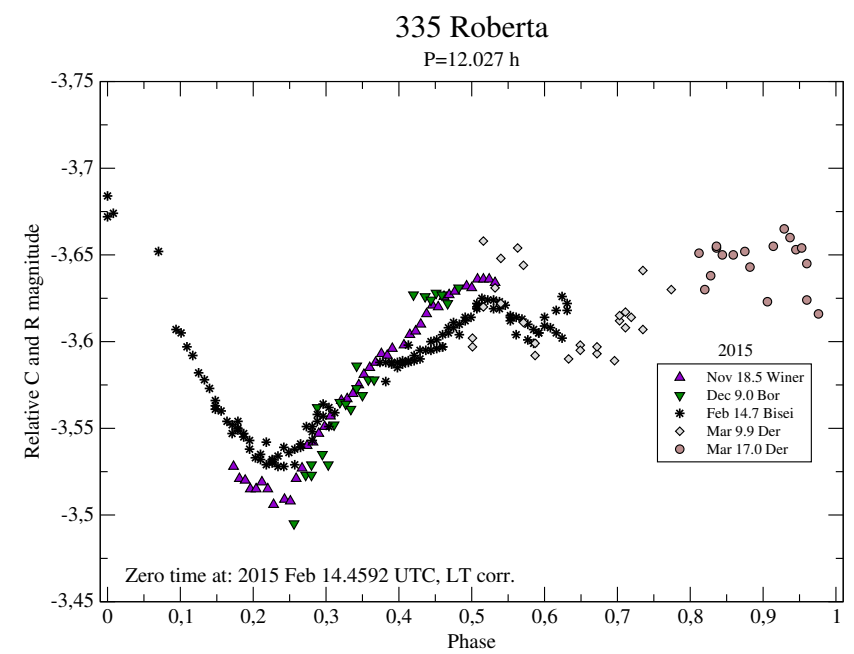

Fig. A.20. Composite lightcurve of (335) Roberta from the year 2015.

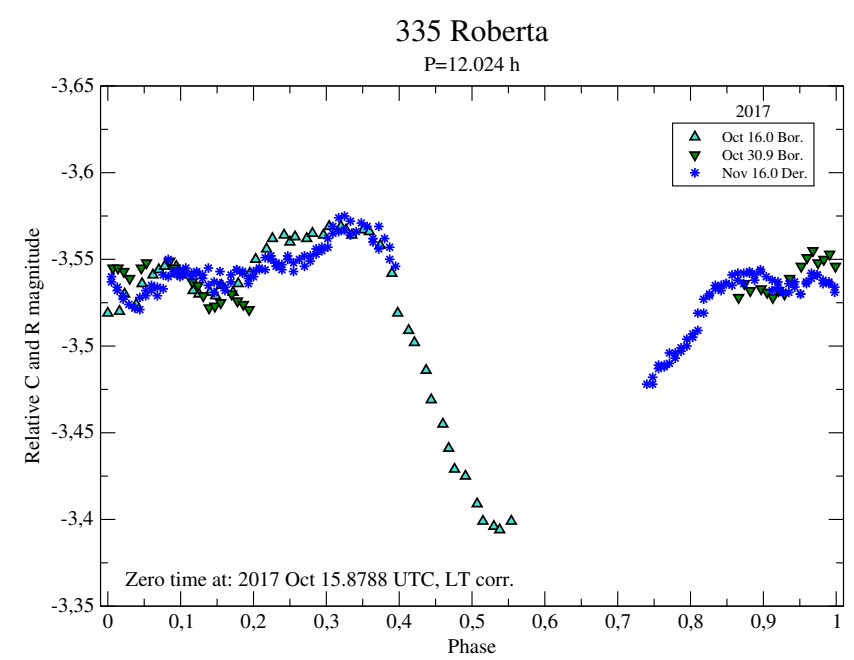

Fig. A.21. Composite lightcurve of (335) Roberta from the year 2017.

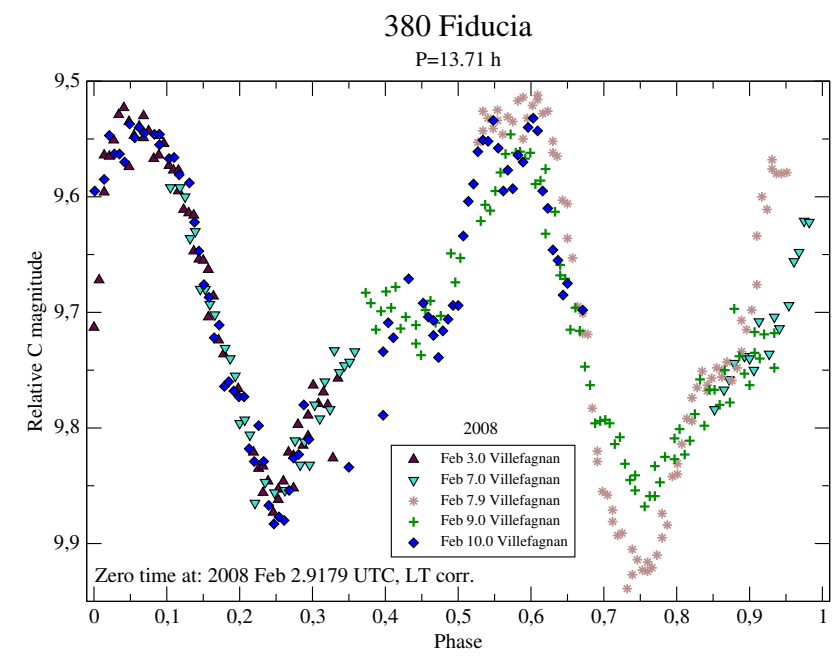

Fig. A.22. Composite lightcurve of (380) Fiducia from the year 2008.

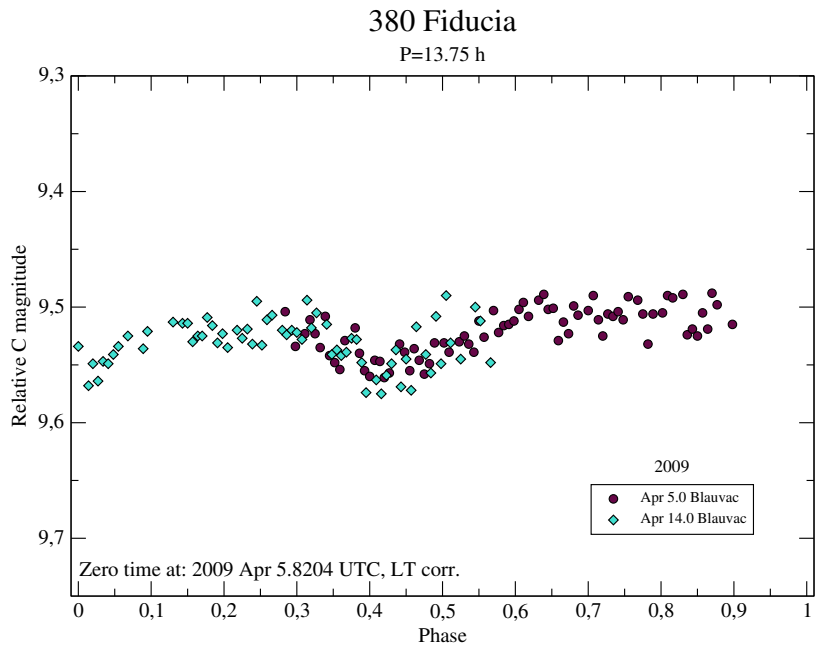

Fig. A.23. Composite lightcurve of (380) Fiducia from the year 2009.

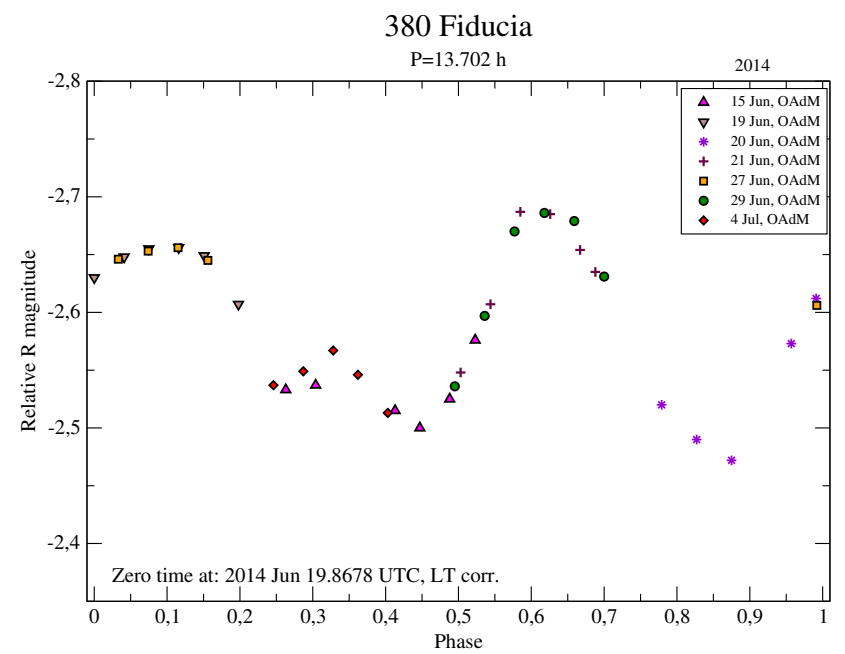

Fig. A.24. Composite lightcurve of (380) Fiducia from the year 2014. 


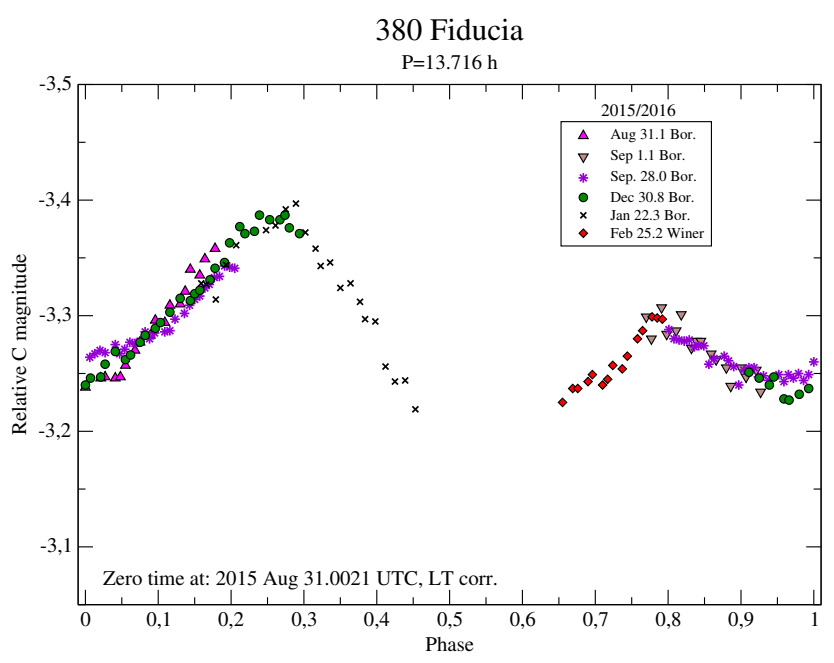

Fig. A.25. Composite lightcurve of (380) Fiducia from the years 20152016 under large phase angle.

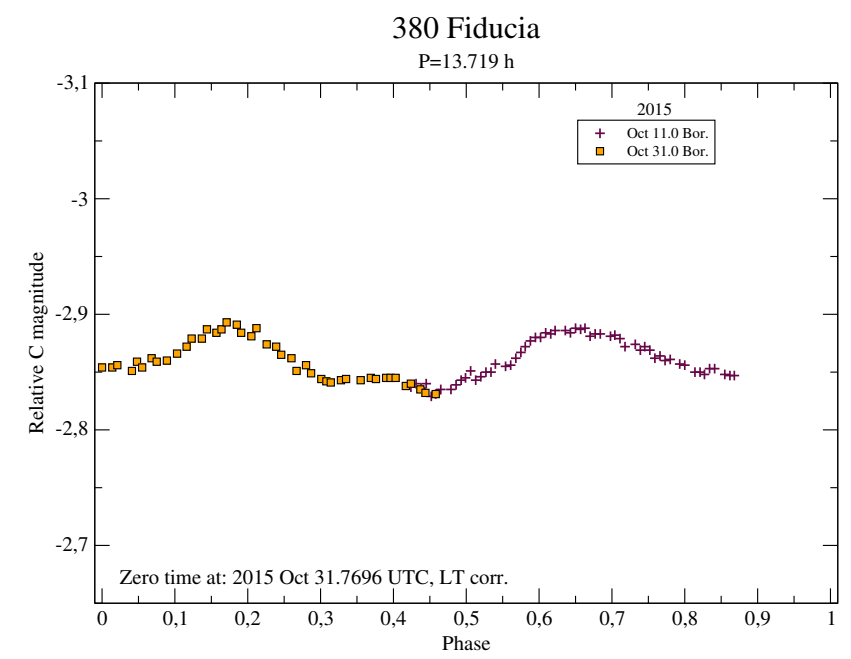

Fig. A.26. Composite lightcurve of (380) Fiducia from the year 2015 under small phase angle.

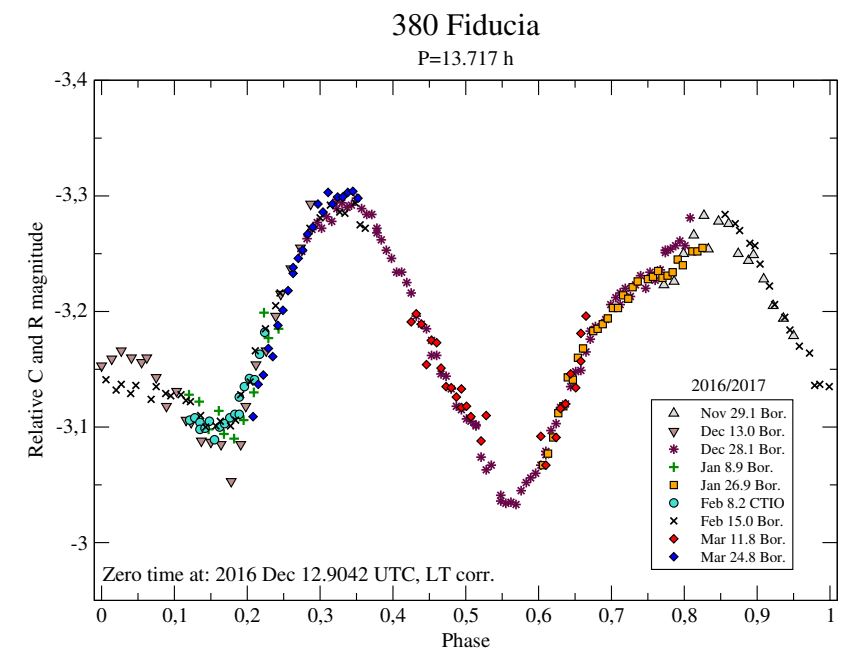

Fig. A.27. Composite lightcurve of (380) Fiducia from the years 20162017.

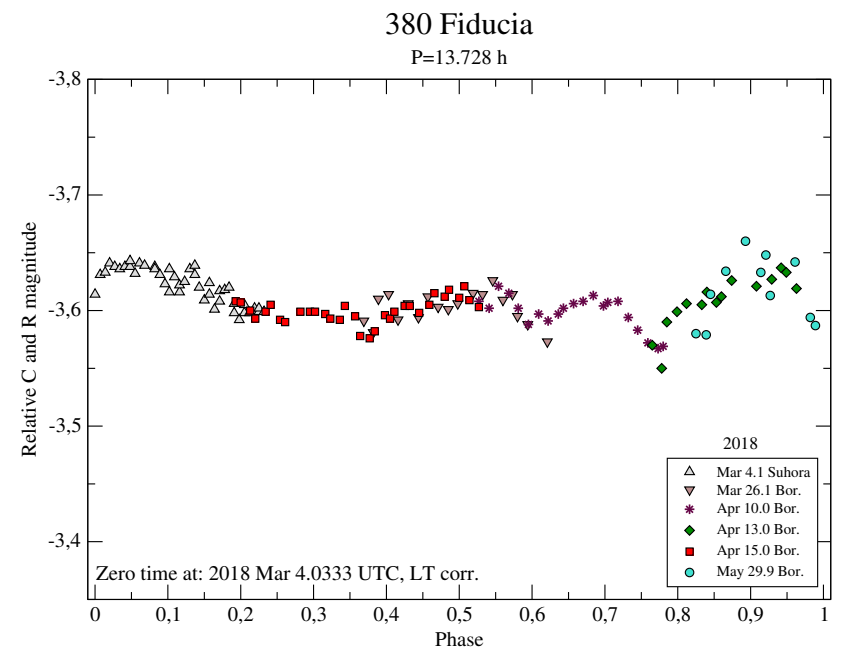

Fig. A.28. Composite lightcurve of (380) Fiducia from the year 2018.

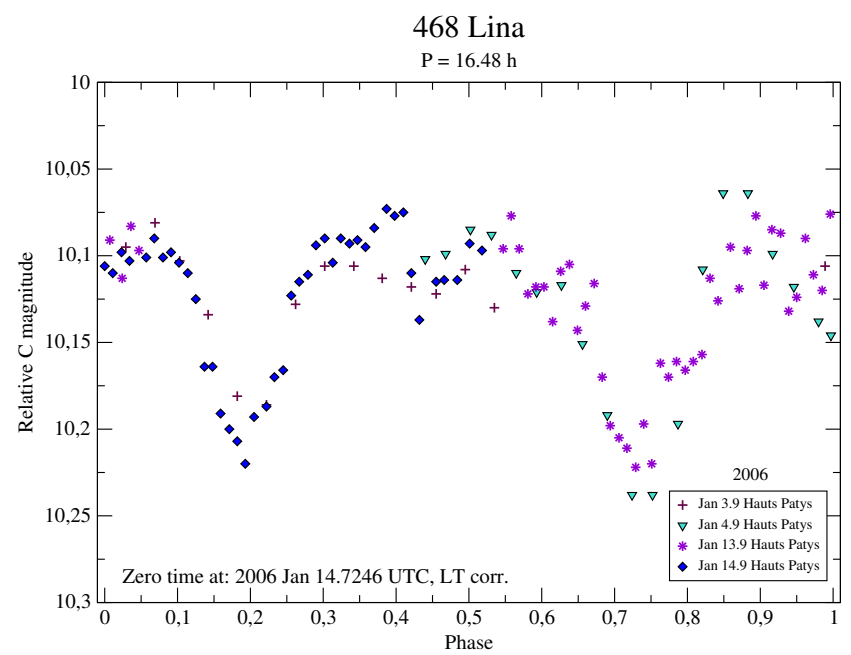

Fig. A.29. Composite lightcurve of (468) Lina from the year 2006.

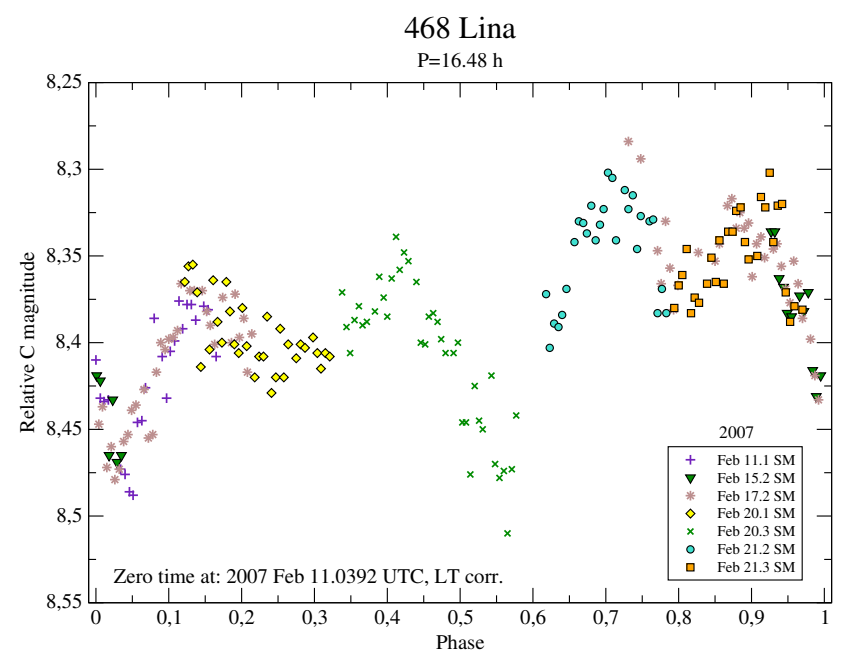

Fig. A.30. Composite lightcurve of (468) Lina from the year 2007. 


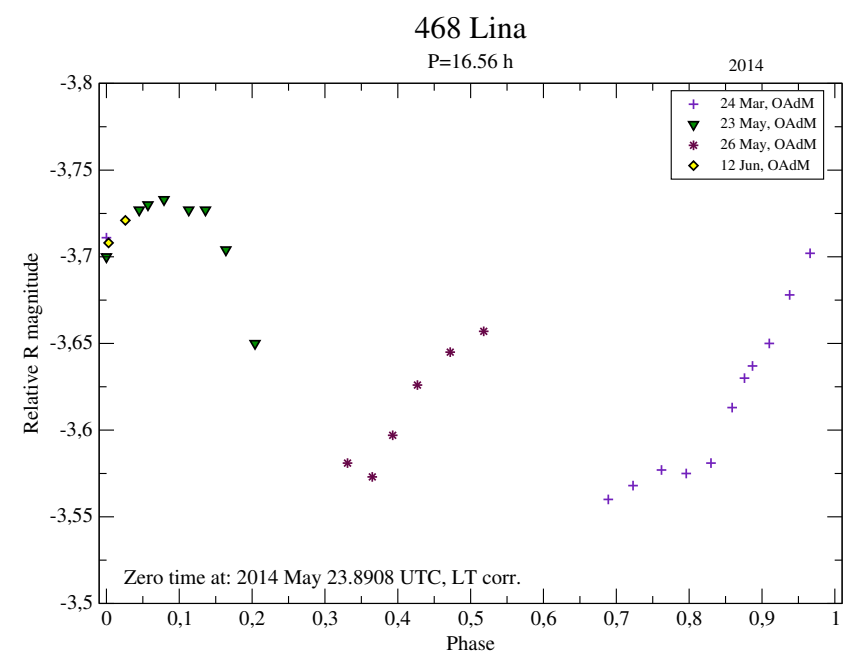

Fig. A.31. Composite lightcurve of (468) Lina from the year 2014.

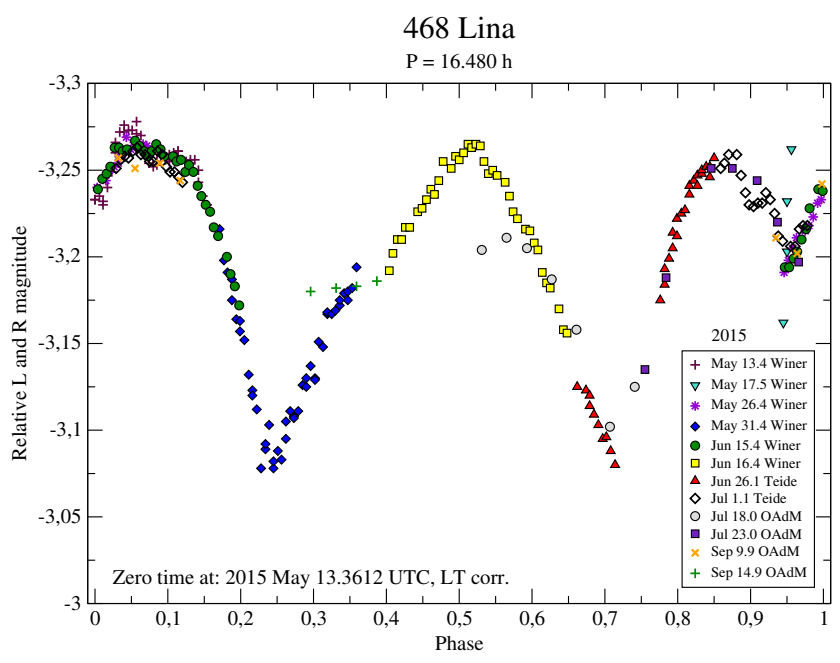

Fig. A.32. Composite lightcurve of (468) Lina from the year 2015.

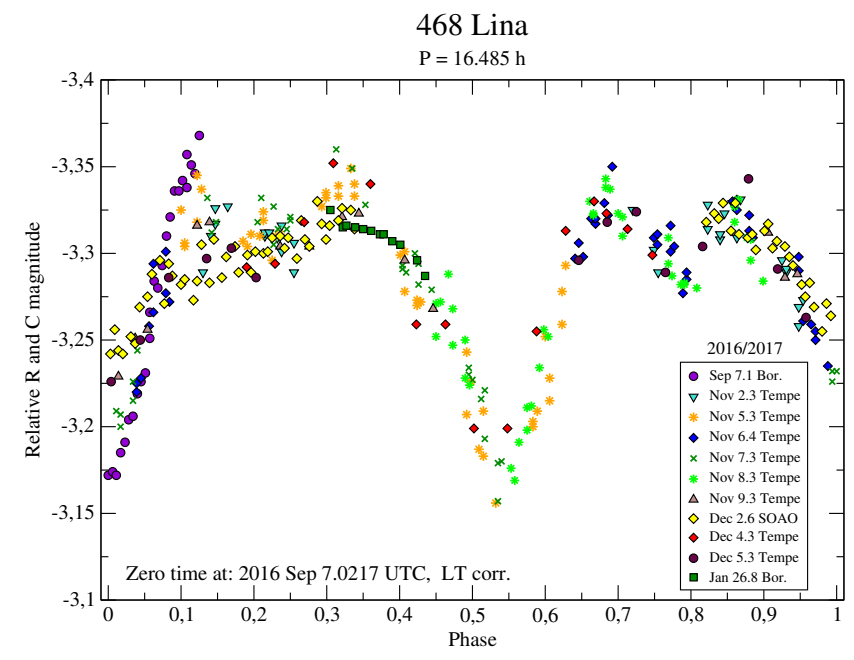

Fig. A.33. Composite lightcurve of (468) Lina from the years 20162017.

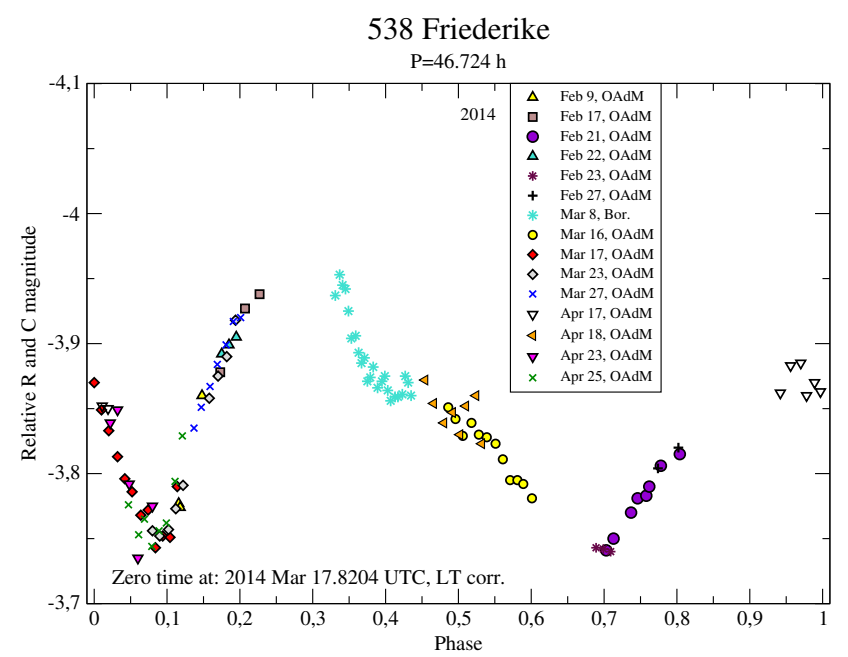

Fig. A.34. Composite lightcurve of (538) Friederike from the year 2014.

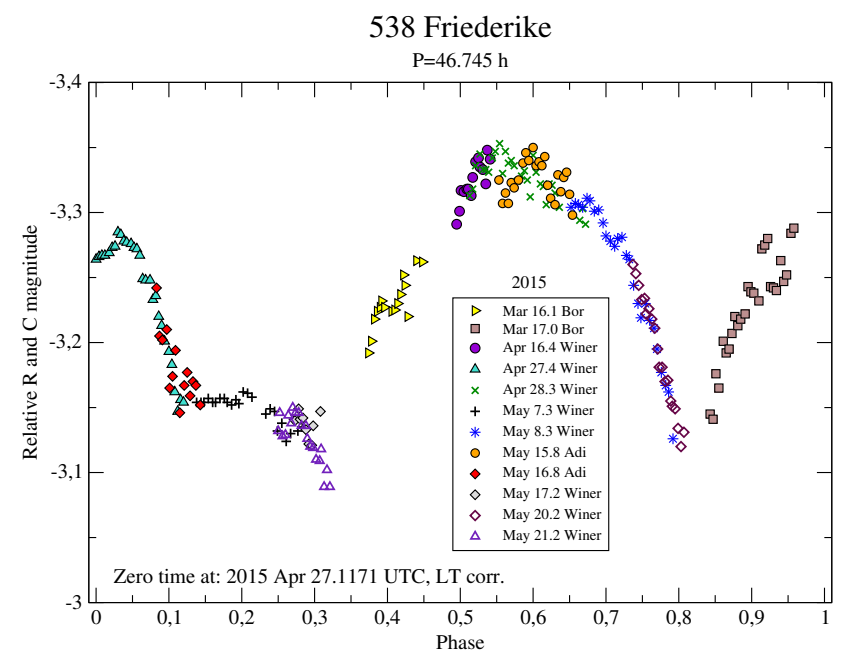

Fig. A.35. Composite lightcurve of (538) Friederike from the year 2015.

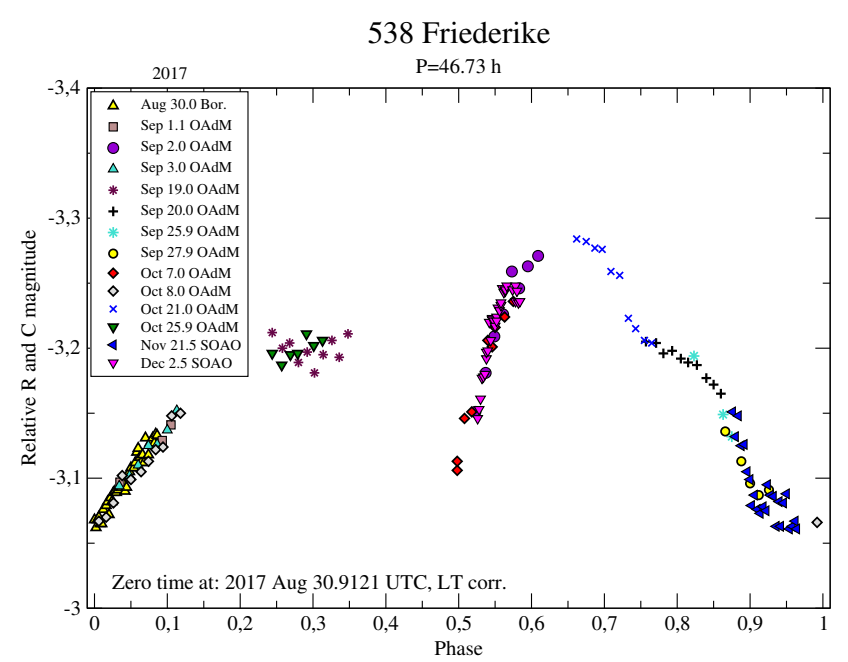

Fig. A.36. Composite lightcurve of (538) Friederike from the year 2017. 


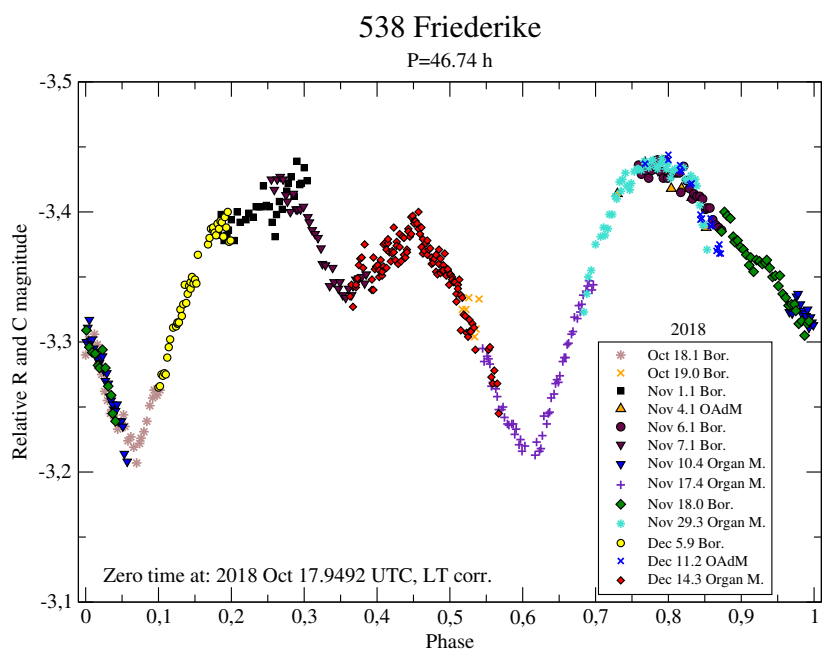

Fig. A.37. Composite lightcurve of (538) Friederike from the year 2018.

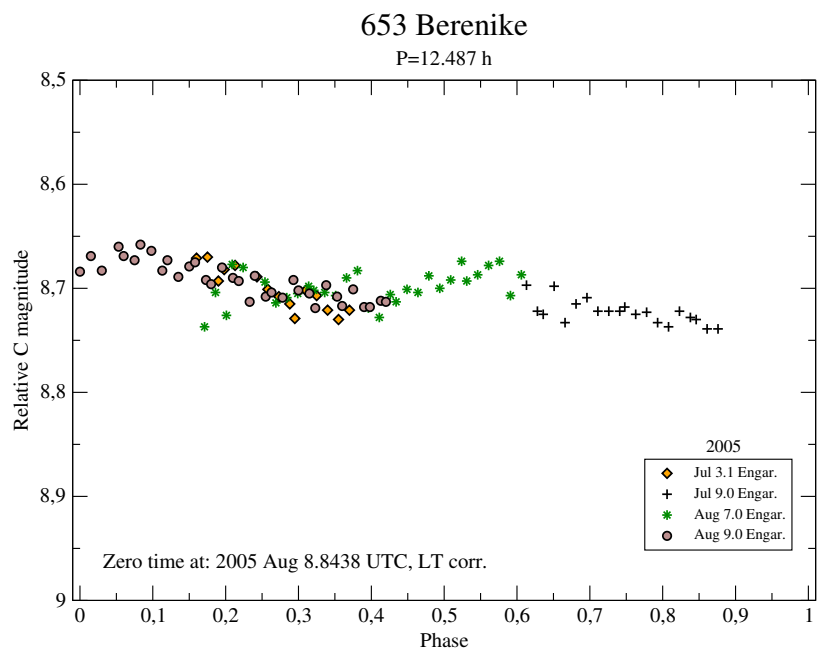

Fig. A.38. Composite lightcurve of (653) Berenike from the year 2005.

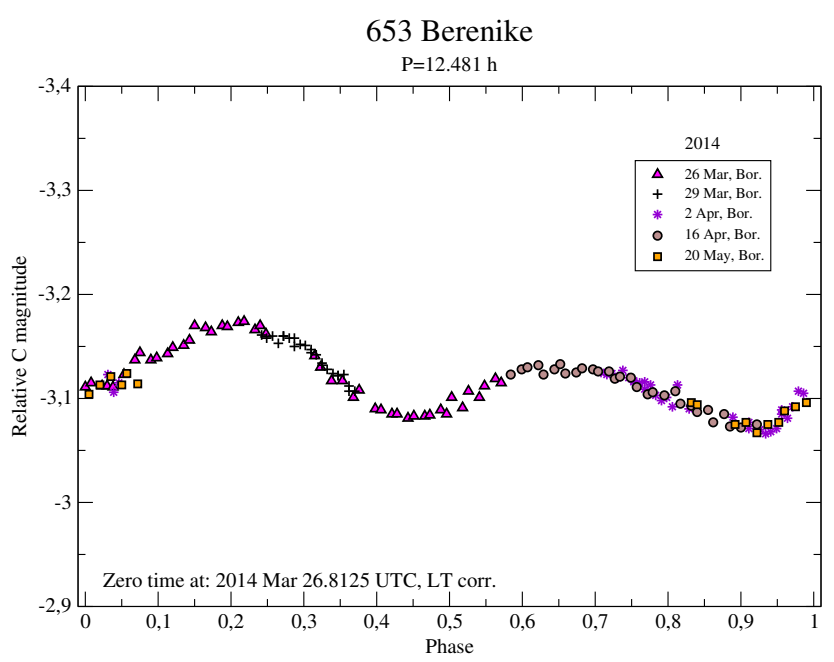

Fig. A.39. Composite lightcurve of (653) Berenike from the year 2014.

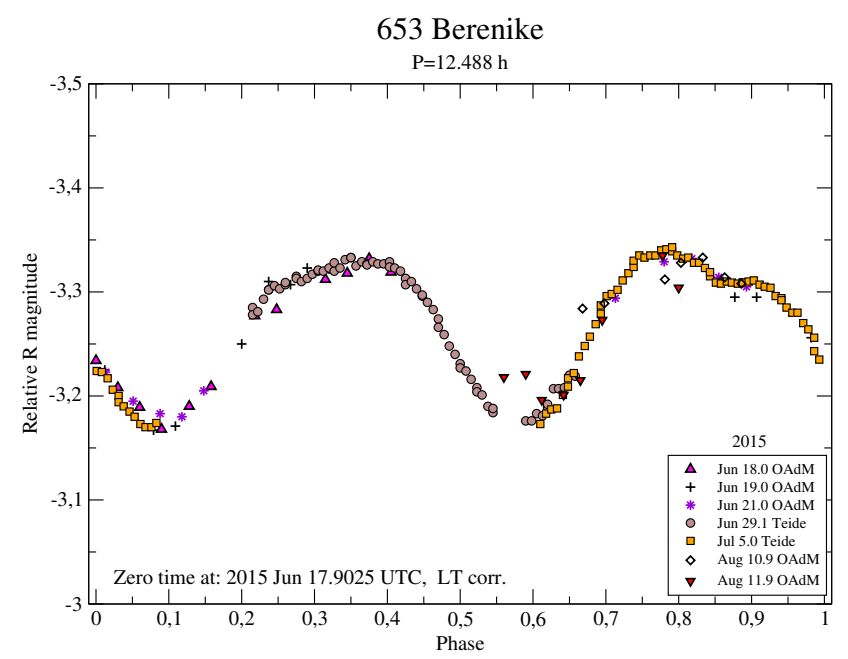

Fig. A.40. Composite lightcurve of (653) Berenike from the year 2015.

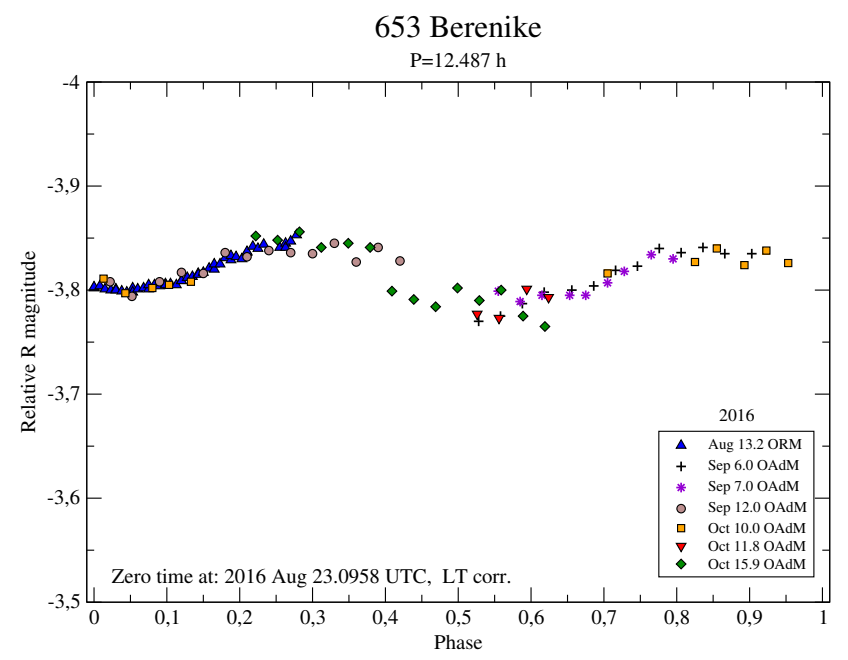

Fig. A.41. Composite lightcurve of (653) Berenike from the year 2016.

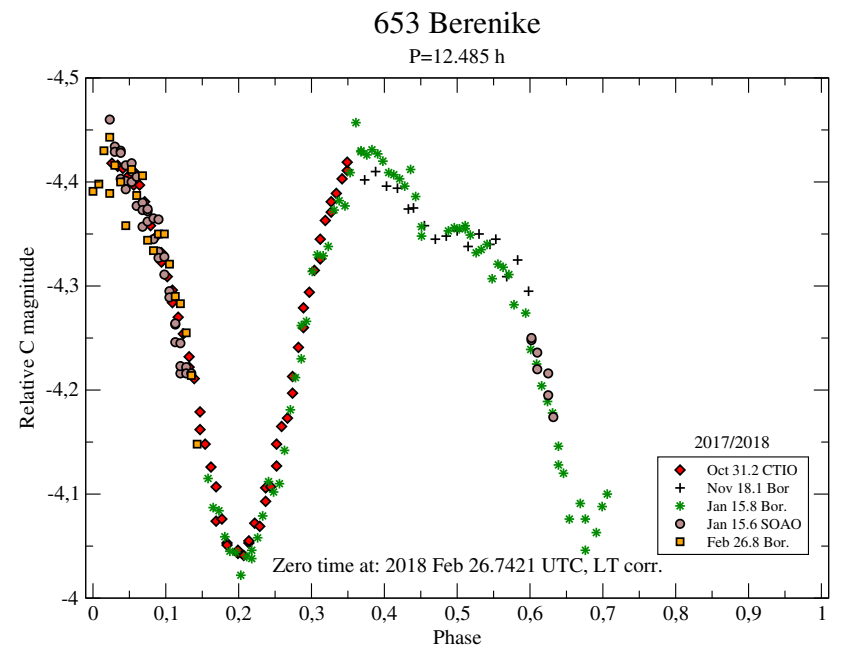

Fig. A.42. Composite lightcurve of (653) Berenike from the years 2017 2018. 


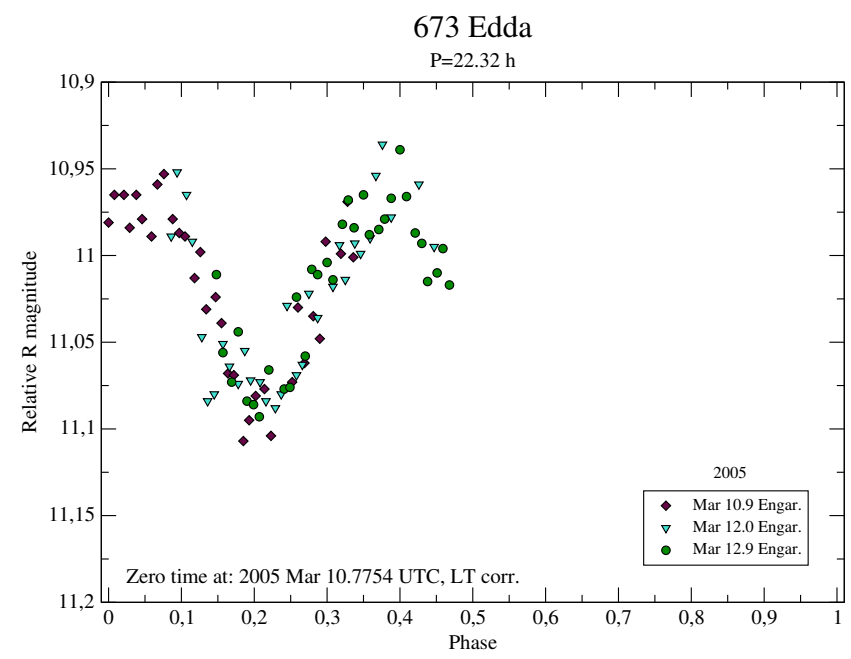

Fig. A.43. Composite lightcurve of (673) Edda from the year 2005.

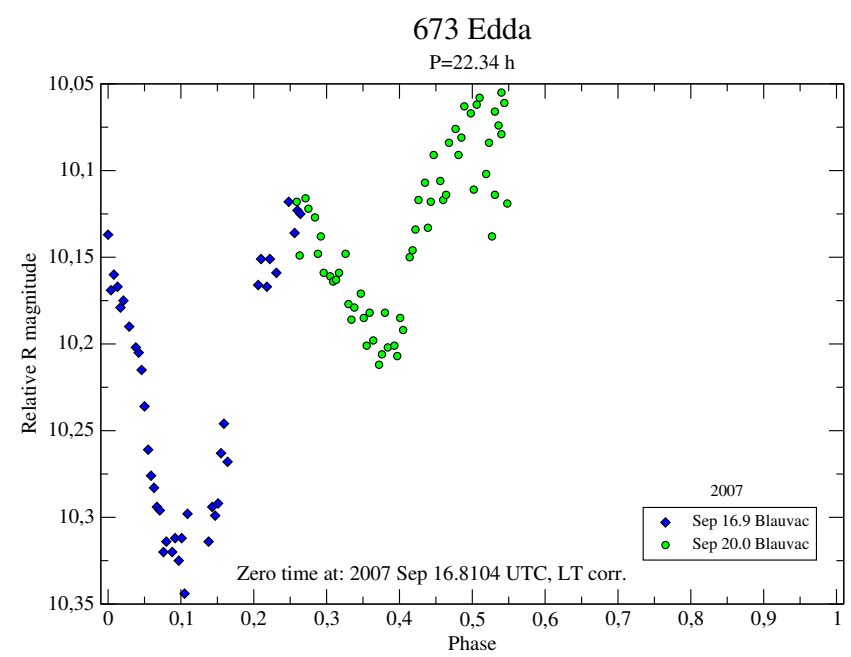

Fig. A.44. Composite lightcurve of (673) Edda from the year 2007.

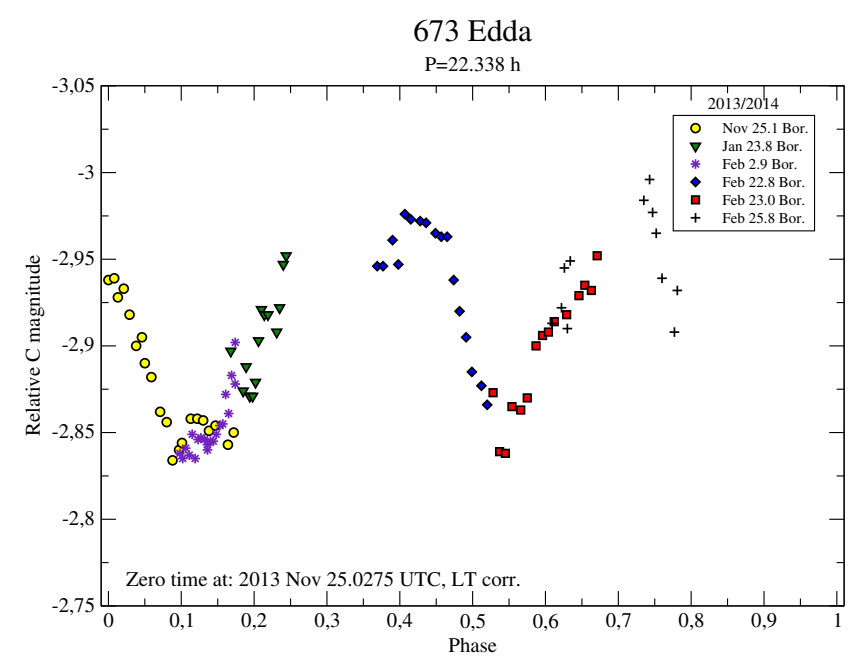

Fig. A.45. Composite lightcurve of (673) Edda from the years 20132014.

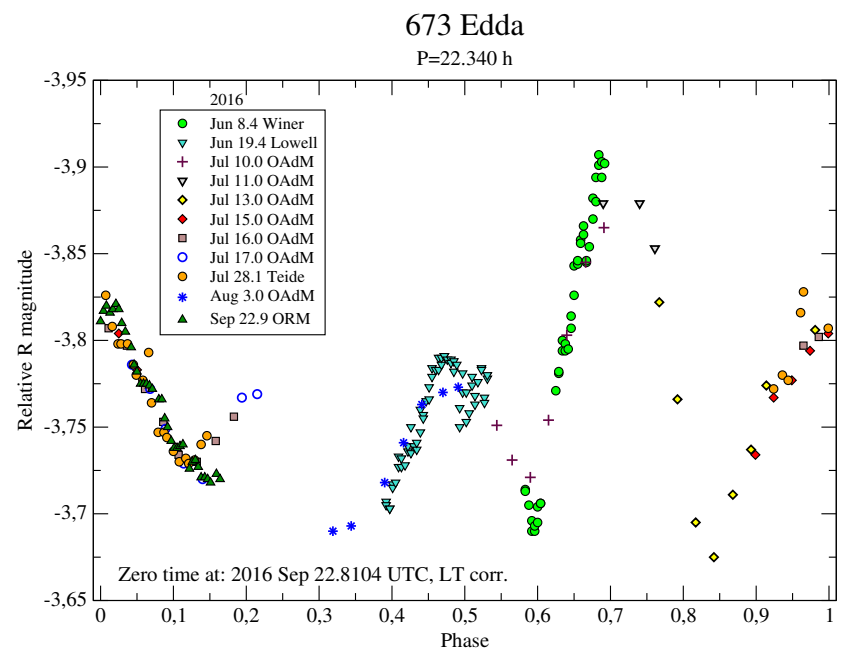

Fig. A.46. Composite lightcurve of (673) Edda from the year 2016.

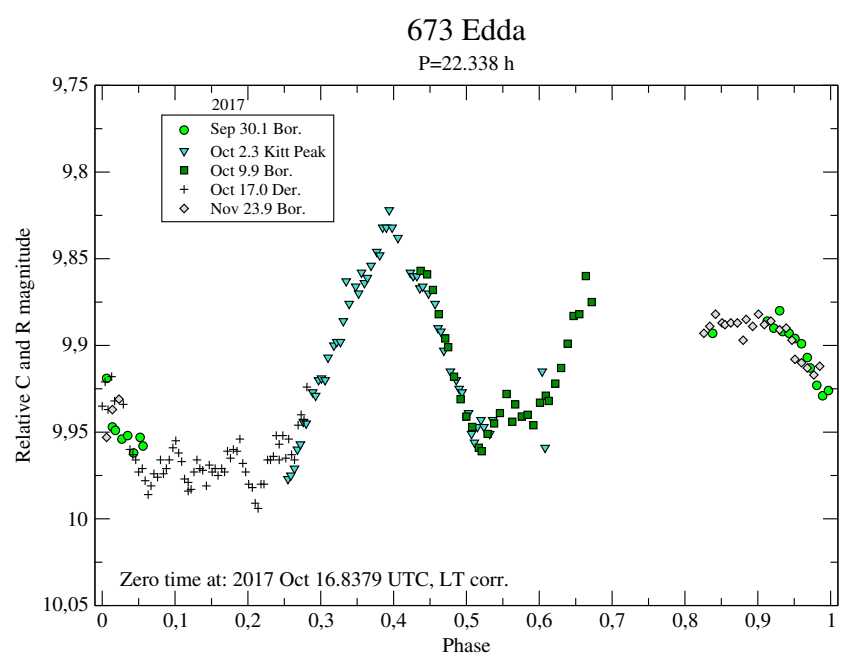

Fig. A.47. Composite lightcurve of (673) Edda from the year 2017.

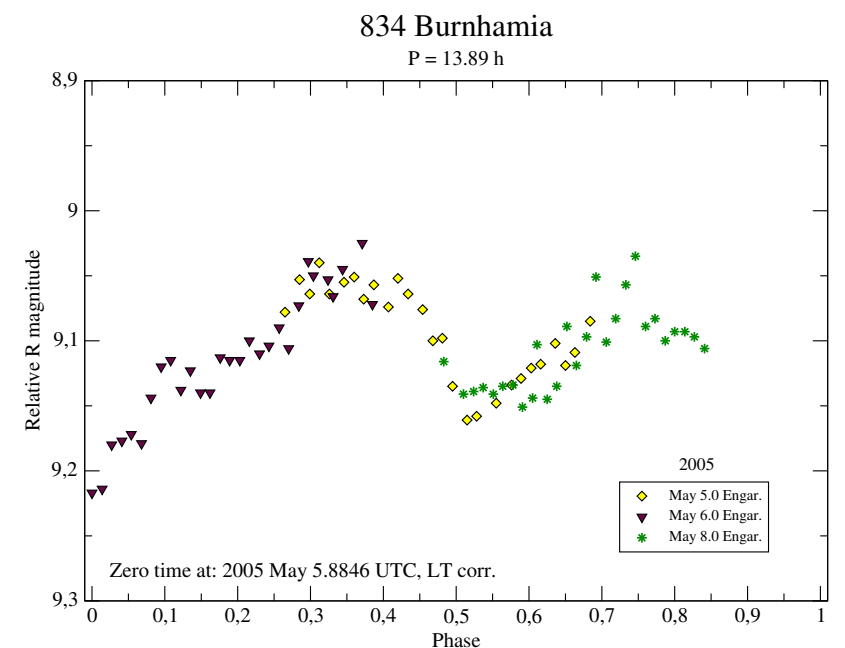

Fig. A.48. Composite lightcurve of (834) Burnhamia from the year 2005 . 


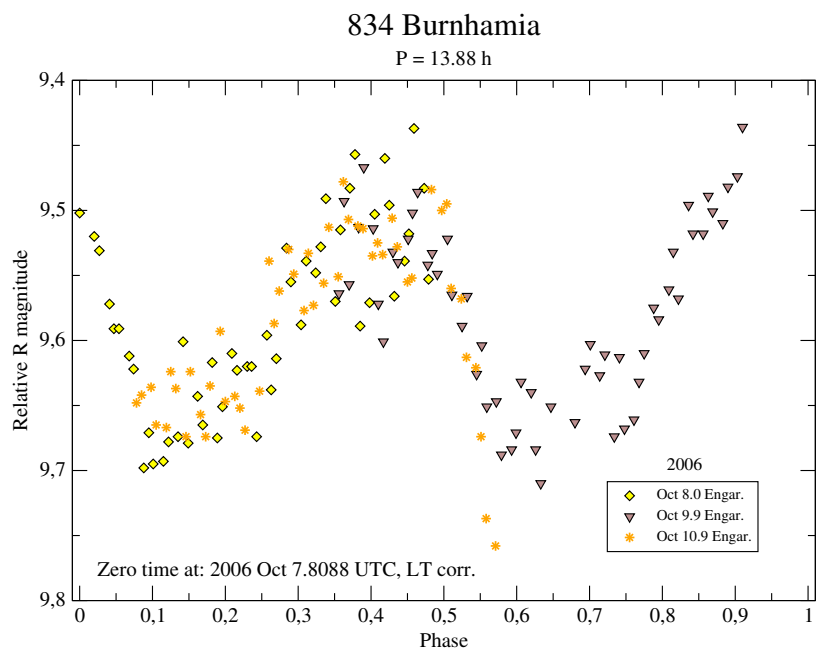

Fig. A.49. Composite lightcurve of (834) Burnhamia from the year 2006.

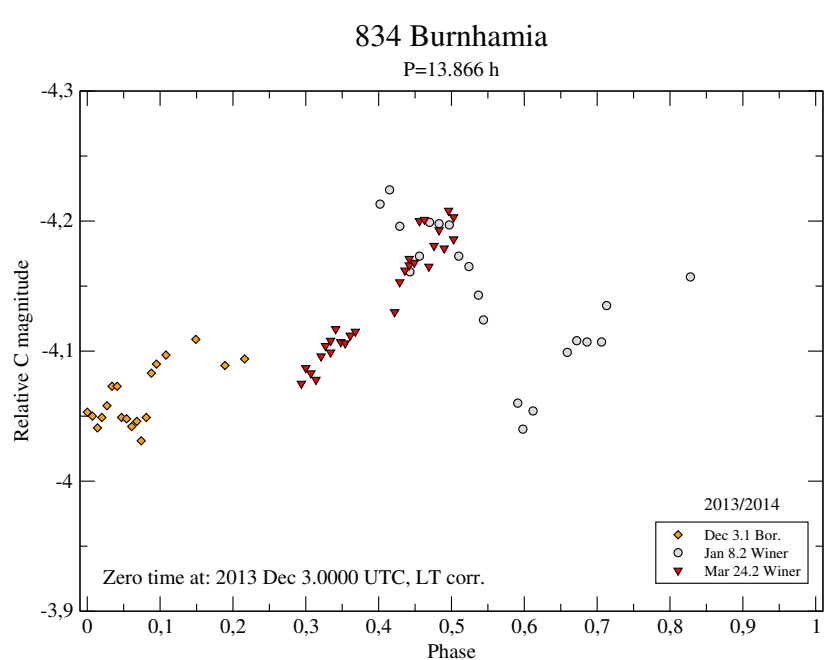

Fig. A.50. Composite lightcurve of (834) Burnhamia from the years 2013-2014.

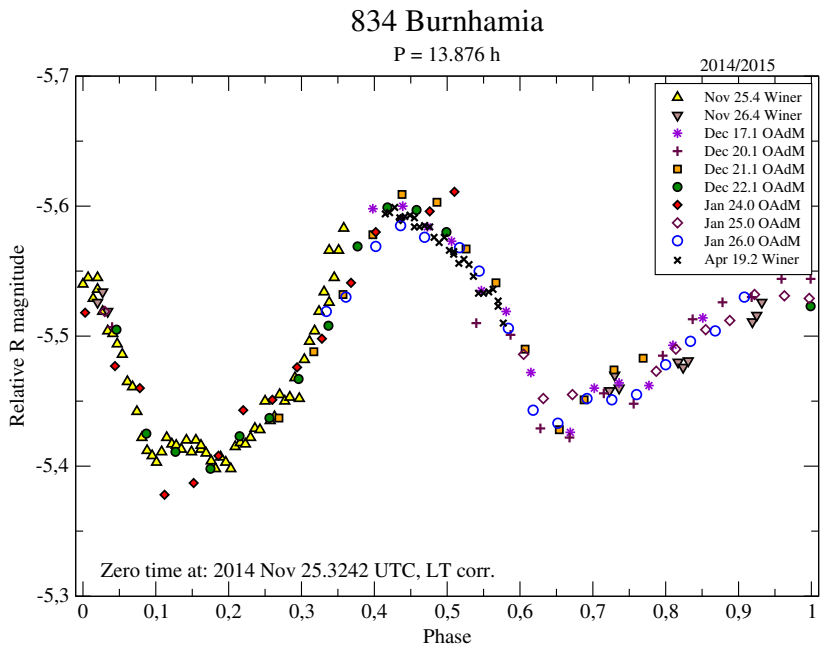

Fig. A.51. Composite lightcurve of (834) Burnhamia from the years 2014-2015.

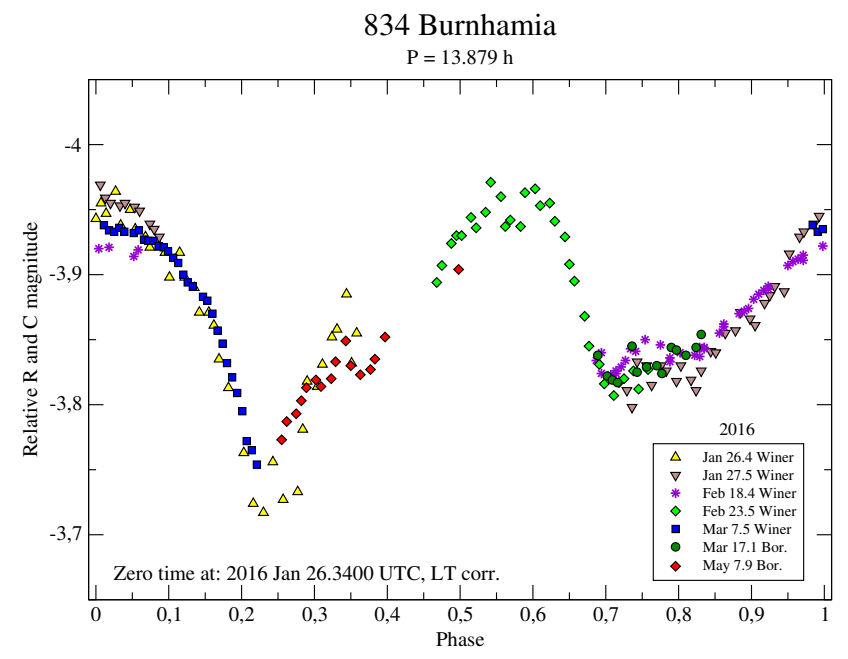

Fig. A.52. Composite lightcurve of (834) Burnhamia from the year 2016.

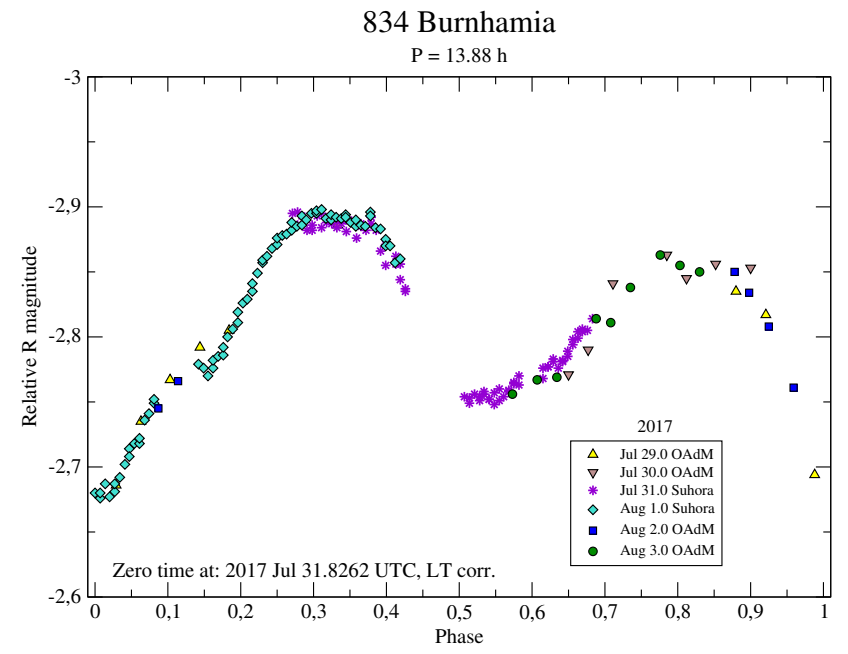

Fig. A.53. Composite lightcurve of (834) Burnhamia from the year 2017. 


\section{Appendix B: Thermophysical model details}

In Sect. 3 in the main text, we provided an overview of our modelling approach. Below, we give a few more details about how we determine the Bond albedo $(A)$ for the TPM and how we estimate our error bars. We also include all the tables (Tables B.1-B.11), observations-to-model-ratio plots (Figs. B.9-B.20), and $\chi^{2}$ versus $\Gamma$ plots that we used to give full information on the TPM analysis.

We fix the value of the Bond albedo to run the model (let us call it $A_{i}$ ). Our TPM diameters $(D)$ do not always lead to the same value of Bond albedo (say, $A_{0}$ ) when we use the $H-G_{12}$ values. However, the differences are not large enough to be meaningful in practice as long as $A \sim 0.1$ or lower. This is because flux is proportional to $T^{4}$, and that in turn is proportional to $(1-A)$, so for example wrongly assuming $A=0.05$ instead of $A=0.10$ would lead to a $\sim 5 \%$ offset in the flux, which is still comparable to the absolute calibration uncertainties. On the other hand, the effect is indeed strong for objects with higher $A$ (e.g. Vesta, with $A \sim 0.2$ ).

To give numbers in our case, all our targets - except one have $A \mathrm{~s}$ in the range $0.02-0.07$, and the differences between $A_{i}$ and $A_{\mathrm{o}}$ never lead to systematic model flux differences $>0.5 \%$, so the effect is negligible. For our highest albedo target, (100) Hekate, we used $A_{i}=0.10$ but got $A_{\mathrm{o}}=0.12$. This $20 \%$ offset leads nonetheless to a $\sim 2 \%$ systematic difference in the model fluxes, which is not ideal but it is well within the absolute calibration flux uncertainties. More quantitatively, we re-ran the TPM for model 2 with $A_{i}=0.12$ and got virtually the same size and thermal inertia but a higher surface roughness $(\mathrm{rms}=0.45$ instead of rms $=0.4$ ). In this case, the slightly lower temperature due to the higher albedo seems to be compensated by a small increase in the roughness.

To estimate $D$ and $\Gamma$ error bars we have followed standard procedures (more details and discussion can be found in Alí-Lagoa et al. 2014; Hanuš et al. 2015). Namely, if we have $\bar{\chi}_{\text {min }}^{2} \approx 1$, the $3 \sigma$ error bars are given by the range of $\Gamma$ s and $D$ s of those models with $\bar{\chi}_{\text {min }}^{2}<3 \sqrt{2 / v}$, where $v$ is the effective number of degrees of freedom (e.g. Press et al. 1986). When $\bar{\chi}_{\text {min }}^{2}$ is much lower than 1 or up to 1.5 , we assume we can scale the $\chi^{2}$ curves to have the minimum at 1 and apply the same formula. This is not rigourous mathematically but it is a working assumption to have some estimate of the error bars (Hanuš et al. 2015). After all, a very low $\bar{\chi}_{\text {min }}^{2}$ could be due to some error bars being overestimated (the IRAS data have the largest ones). If $\bar{\chi}_{\min }^{2}$ is about 1.5 , we assume it is still a reasonable fit given the model simplification (constant parameters over the surface, wavelength-independent emissivity) or other sources of errors. For instance, we do not consider the uncertainties in shape (no shape modelling scheme so far provides shape uncertainties), rotation period, or spin orientation explicitly. These are coupled parameters themselves in the shape modelling so we cannot simply vary them separately within their uncertainties to explore the effects in TPM. Hanuš et al. (2015) proposed bootstrapping the visible photometry to obtain $\sim 30$ shape and spin models that were subsequently input to the TPM (the socalled varied-shape TPM). The values of acceptable $\Gamma$ s spanned were significantly larger than the classical approach only in some cases, not systematically.

Table B.1. Summary of TPM results for (100) Hekate.

\begin{tabular}{|c|c|c|c|c|c|c|}
\hline Shape model & IR data subset & $\bar{\chi}_{m}^{2}$ & $D \pm 3 \sigma(\mathrm{km})$ & $\Gamma \pm 3 \sigma(\mathrm{SIu})$ & Roughness (rms) & Comments \\
\hline AM 1 & All data & 0.8 & $90_{-4}^{+6}$ & $6_{-4}^{+114}$ & Low/medium (0.30) & rms unconstrained at the $3 \sigma$ level \\
\hline AM 1 sphere & All data & 0.8 & $86_{-5}^{+6}$ & $20_{-20}^{+160}$ & Medium/high (0.6) & rms unconstrained at the $3 \sigma$ level \\
\hline AM 2 & All data & 0.65 & $87_{-4}^{+5}$ & $4_{-2}^{+66}$ & Medium (0.40) & rms unconstrained at the $3 \sigma$ level \\
\hline AM 2 sphere & All data & 0.8 & $86_{-8}^{+8}$ & $16_{-16}^{+144}$ & Medium/high $(\sim 0.6)$ & rms unconstrained at the $3 \sigma$ level \\
\hline
\end{tabular}

Table B.2. Summary of TPM results for (109) Felicitas.

\begin{tabular}{llcccll}
\hline \hline Shape model & IR data subset & $\bar{\chi}_{m}^{2}$ & $D \pm 3 \sigma(\mathrm{km})$ & $\Gamma \pm 3 \sigma(\mathrm{SIu})$ & Roughness $(\mathrm{rms})$ & Comments \\
\hline AM 1 & All data & 1.1 & $85_{-5}^{+7}$ & $40_{-40}^{+100}$ & Ext. high $(\sim 1.0)$ & rms unconstrained at the $3 \sigma$ level \\
AM 1 sphere & All data & 2.2 & 97 & 200 & Ext. high $(\sim 1.0)$ & Bad fit \\
\hline AM 2 & All data & 2.0 & 81 & 4 & Ext. high $(\sim 1.0)$ & Bad fit \\
AM 2 sphere & All data & 3.4 & 101 & 250 & Ext. high $(\sim 1.0)$ & Bad fit \\
\hline
\end{tabular}


A. Marciniak et al.: Thermal properties of slowly rotating asteroids

Table B.3. Summary of TPM results for (195) Eurykleia fitting different subsets of data.

\begin{tabular}{|c|c|c|c|c|c|c|}
\hline Shape model & IR data subset & $\bar{\chi}_{m}^{2}$ & $D \pm 3 \sigma(\mathrm{km})$ & $\Gamma \pm 3 \sigma(\mathrm{SIu})$ & Roughness (rms) & Comments \\
\hline AM 1 & All data & 0.51 & $82_{-4}^{+5}$ & $15_{-15}^{+37}$ & High (0.65) & $\begin{array}{l}\text { rms }>0.34 \text { at the } 1 \sigma \text { level, } \\
\text { but otherwise unconstrained }\end{array}$ \\
\hline AM 1 sphere & All data & 1.23 & $83_{-4}^{+7}$ & $25_{-20}^{+65}$ & Extr. high $(>1.0)$ & Roughness unconstrained \\
\hline AM 1 & Excluding W4 & 0.50 & $87_{-6}^{+5}$ & $15_{-15}^{+35}$ & Medium (0.45) & Roughness unconstrained \\
\hline AM 1 & Only W4 & 0.23 & 81 & 5 & Low (0.29) & $\begin{array}{l}\text { Artificially low } \bar{\chi}_{\mathrm{m}}^{2} \text {. Single-epoch data } \\
\text { are insufficient to constrain params. }\end{array}$ \\
\hline AM 2 & All data & 0.60 & $86_{-4}^{+5}$ & $20_{-20}^{+40}$ & Extr. high $(\sim 1.0)$ & $\begin{array}{l}\text { Roughness rms }>0.34 \\
\text { at the } 3 \sigma \text { level }\end{array}$ \\
\hline AM 2 sphere & All data & 1.17 & $84_{-3}^{+8}$ & $30_{-25}^{+60}$ & Extr. high $(>1.0)$ & Roughness unconstrained \\
\hline AM 2 & Excluding W4 & 0.58 & $91_{-6}^{+7}$ & $15_{-15}^{+50}$ & Med.-high (0.5) & Fitted with rel. calibration error bars \\
\hline AM 2 & Only W4 & 0.47 & 80 & 5 & Extr. high $(>1.0)$ & $\begin{array}{l}\text { Artificially low } \bar{\chi}_{\mathrm{m}}^{2} \text {. Single-epoch data } \\
\text { are insufficient to constrain params. }\end{array}$ \\
\hline
\end{tabular}

Notes. For example, the "Excluding W4" label refers to the fact that the W4 data were not used to optimise the $\chi^{2}$ in that case.

Table B.4. Summary of TPM results for (301) Bavaria.

\begin{tabular}{|c|c|c|c|c|c|c|}
\hline Shape model & IR data subset & $\bar{\chi}_{m}^{2}$ & $D \pm 3 \sigma(\mathrm{km})$ & $\Gamma \pm 3 \sigma(\mathrm{SIu})$ & Roughness (rms) & Comments \\
\hline AM 1 & All data & 0.34 & $55_{-2}^{+2}$ & $40_{-30}^{+60}$ & Med.-high (0.5) & rms unconstrained at the $3 \sigma$ level \\
\hline AM 1 sphere & All data & 0.7 & $57_{-3}^{+3}$ & $30_{-25}^{+90}$ & Low (0.20) & rms unconstrained at the $3 \sigma$ level \\
\hline AM 2 & All data & 0.36 & $55_{-2}^{+2}$ & $50_{-35}^{+50}$ & Medium/high (0.60) & rms unconstrained at the $3 \sigma$ level \\
\hline AM 2 sphere & All data & 0.7 & $57_{-3}^{+3}$ & $30_{-25}^{+70}$ & Low $(0.20)$ & rms unconstrained at the $3 \sigma$ level \\
\hline
\end{tabular}

Table B.5. Summary of TPM results for (335) Roberta.

\begin{tabular}{llccccl}
\hline \hline Shape model & IR data subset & $\bar{\chi}_{m}^{2}$ & $D \pm 3 \sigma(\mathrm{km})$ & $\Gamma \pm 3 \sigma(\mathrm{SIu})$ & Roughness $(\mathrm{rms})$ & Comments \\
\hline AM 1 & All data & 0.70 & $100_{-11}^{+10}$ & $70_{-55}^{+1500}$ & Very low $(0.21)$ & $\begin{array}{l}\text { Roughness and } \Gamma \text { unconstrained } \\
\text { at the } 3 \sigma \text { level } \\
\text { Compatible solution but requires } \\
\text { extremely high roughness }\end{array}$ \\
\hline AM 1 sphere & All data & 0.71 & $97_{-11}^{+8}$ & $150_{-125}^{+1350}$ & Extr. high $(\sim 1.0)$ & $\begin{array}{l}\text { Roughness and } \Gamma \text { unconstrained } \\
\text { aM 2 }\end{array}$ \\
\hline All data & 0.60 & $98_{-8}^{+10}$ & $90_{-85}^{+1410}$ & Low $(0.34)$ & Extr. high $(\sim 1.0)$ & Very similar to "sphere AM 1" fit \\
\hline
\end{tabular}

Table B.6. Summary of TPM results for (380) Fiducia.

\begin{tabular}{llccccl}
\hline \hline Shape model & IR data subset & $\bar{\chi}_{m}^{2}$ & $D \pm 3 \sigma(\mathrm{km})$ & $\Gamma \pm 3 \sigma(\mathrm{SIu})$ & Roughness $(\mathrm{rms})$ & Comments \\
\hline AM 1 & All data & 0.91 & $73_{-5}^{+8}$ & $15_{-15}^{+135}$ & $\begin{array}{c}\text { Low }(0.25) \\
\text { Extr. high }(\sim 1.0)\end{array}$ & $\begin{array}{l}\text { rms }<0.90 \text { at the } 3 \sigma \text { level } \\
\text { borderline acceptable } \bar{\chi}_{m}^{2}\end{array}$ \\
AM 1 sphere & All data & 1.33 & 78 & 200 & & but unconstrained thermal props. \\
& & & & & Low $(0.25)$ & rms $<0.90$ at the $3 \sigma$ level \\
AM 2 & All data & 0.59 & $72_{-5}^{+9}$ & $10_{-10}^{+140}$ & Extr. high $(\sim 1.0)$ & $\begin{array}{l}\text { Borderline acceptable } \bar{\chi}_{m}^{2} \\
\text { but unconstrained thermal props. }\end{array}$ \\
\hline
\end{tabular}


Table B.7. Summary of TPM results for (468) Lina.

\begin{tabular}{llccccl}
\hline \hline Shape model & IR data subset & $\bar{\chi}_{m}^{2}$ & $D \pm 3 \sigma(\mathrm{km})$ & $\Gamma \pm 3 \sigma(\mathrm{SIu})$ & Roughness $(\mathrm{rms})$ & Comments \\
\hline AM 1 & All data & 1.20 & $69_{-4}^{+11}$ & $20_{-20}^{+280}$ & Very low $(0.20)$ & $\begin{array}{l}\text { rms unconstrained at the } 3 \sigma \text { level } \\
\text { Low }(\sim 0.30)\end{array}$ \\
AM 1 sphere & All data & 2.0 & 65 & 35 & $\begin{array}{l}\text { Bad } \bar{\chi}_{m}^{2} \text { but } \\
\text { similar thermal properties }\end{array}$ \\
& & & & & Very low $(0.2)$ & rms not constrained at the $3 \sigma$ level \\
AM 2 & All data & 1.22 & $70_{-5}^{+11}$ & $20_{-20}^{+280}$ & Low $(\sim 0.30)$ & $\begin{array}{l}\text { Bad } \bar{\chi}_{m}^{2} \text { but } \\
\text { similar thermal properties }\end{array}$ \\
\hline
\end{tabular}

Table B.8. Summary of TPM results for (538) Friederike.

\begin{tabular}{llccccl}
\hline \hline Shape model & IR data subset & $\bar{\chi}_{m}^{2}$ & $D \pm 3 \sigma(\mathrm{km})$ & $\Gamma \pm 3 \sigma(\mathrm{SIu})$ & Roughness $(\mathrm{rms})$ & Comments \\
\hline AM 1 & All data & 1.65 & $74_{-1}^{+2}$ & $<15$ & Extr. high $(0.9)$ & Bad fit \\
AM 1 sphere & All data & 1.52 & $71_{-1}^{+3}$ & $<28$ & Extr. high $\sim 1$ & Bad fit \\
AM 1 & WISE only & 0.99 & $73_{-2}^{+3}$ & $10_{-6}^{+34}$ & Extr. high $\sim 1$ & $\mathrm{rms}>0.40$ \\
\hline AM 2 & All data & 2.04 & $75_{-1}^{+2}$ & $<14$ & High $(0.75)$ & Bad fit \\
AM 2 sphere & All data & 1.52 & $71_{-1}^{+3}$ & $<28$ & Extr. high $\sim 1$ & Bad fit \\
AM 2 & WISE only & 0.72 & $76_{-2}^{+5}$ & $20_{-20}^{+25}$ & Extr. high $\sim 1$ & $\mathrm{rms}>0.45$ \\
\hline
\end{tabular}

Table B.9. Summary of TPM results for (653) Berenike.

\begin{tabular}{llccccl}
\hline \hline Shape model & IR data subset & $\bar{\chi}_{m}^{2}$ & $D \pm 3 \sigma(\mathrm{km})$ & $\Gamma \pm 3 \sigma(\mathrm{SIu})$ & Roughness (rms) & Comments \\
\hline AM 1 & All data & 1.1 & $46_{-2}^{+4}$ & $40_{-40}^{+120}$ & Med.-high $(0.5)$ & rms $<0.3$ rejected at the $3 \sigma$ level \\
AM 1 sphere & All data & 2.0 & 52 & 100 & Extr. high (1.00) & Bad fit \\
\hline AM 2 & All data & 2.0 & 52 & 120 & Extr. high (1.00) & Bad fit \\
AM 2 sphere & All data & 2.6 & 52 & 100 & Extr. high (1.00) & Bad fit \\
\hline
\end{tabular}

Table B.10. Summary of TPM results for (673) Edda.

\begin{tabular}{llccccl}
\hline \hline Shape model & IR data subset & $\bar{\chi}_{m}^{2}$ & $D \pm 3 \sigma(\mathrm{km})$ & $\Gamma \pm 3 \sigma(\mathrm{SIu})$ & Roughness $(\mathrm{rms})$ & Comments \\
\hline AM 1 & All data & 0.47 & $38_{-2}^{+6}$ & $3_{-3}^{+33}$ & Med.-high $(0.5)$ & rms $>0.34$ at the $3 \sigma$ level \\
AM 1 sphere & All data & 1.83 & 38 & 5 & Medium (0.4) & Bad fit \\
\hline AM 2 & All data & 0.59 & $38_{-2}^{+2}$ & $3_{-3}^{+37}$ & Extr. high $(\sim 1.0)$ & rms $>0.34$ at the $3 \sigma$ level \\
AM 2 sphere & All data & 1.76 & 38 & 10. & Medium $(0.45)$ & Bad fit \\
\hline
\end{tabular}

Table B.11. Summary of TPM results for (834) Burnhamia.

\begin{tabular}{llccccl}
\hline \hline Shape model & IR data subset & $\bar{\chi}_{m}^{2}$ & $D \pm 3 \sigma(\mathrm{km})$ & $\Gamma \pm 3 \sigma(\mathrm{SIu})$ & Roughness $(\mathrm{rms})$ & Comments \\
\hline AM 1 & All data & 0.78 & $67_{-6}^{+8}$ & $22_{-22}^{+23}$ & Extr. high $(0.9)$ & rms unconstrained at the $3 \sigma$ level \\
AM 1 sphere & All data & 1.12 & $65_{-5}^{+6}$ & $40_{-40}^{+45}$ & Extr. high $(\sim 1.0)$ & $\begin{array}{l}\text { Acceptable } \bar{\chi}_{m}^{2} \text { and } \\
\text { similar thermal properties }\end{array}$ \\
\hline AM 2 & All data & 0.80 & $66_{-4}^{+5}$ & $20_{-20}^{+30}$ & High $(0.6)$ & rms not constrained at the $3 \sigma$ level \\
AM 2 sphere & All data & 1.12 & $65_{-5}^{+6}$ & $40_{-40}^{+45}$ & Extr. high $(\sim 1.0)$ & $\begin{array}{l}\text { Acceptable } \bar{\chi}_{m}^{2} \text { but } \\
\text { similar thermal properties }\end{array}$ \\
\hline
\end{tabular}


A. Marciniak et al.: Thermal properties of slowly rotating asteroids

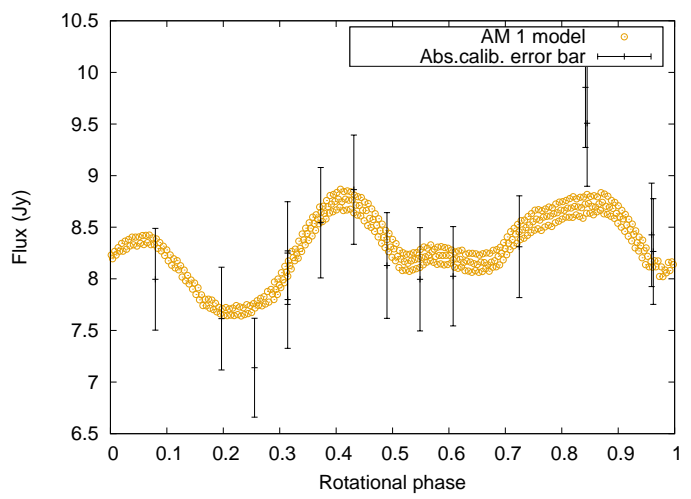

Fig. B.1. Asteroid (100) Hekate W4 data and model thermal lightcurves for shape model 1. Data error bars are $1 \sigma$. Table B.1 summarises the TPM analysis.

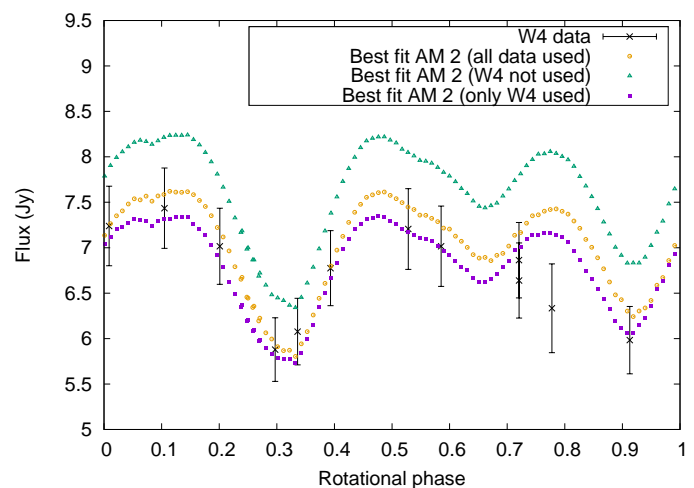

Fig. B.2. W4 data and model thermal lightcurves for (195) Eurykleia's shape model AM 2. The different models resulted from fitting different subsets of data. Table B. 3 contains the corresponding thermo-physical parameters.

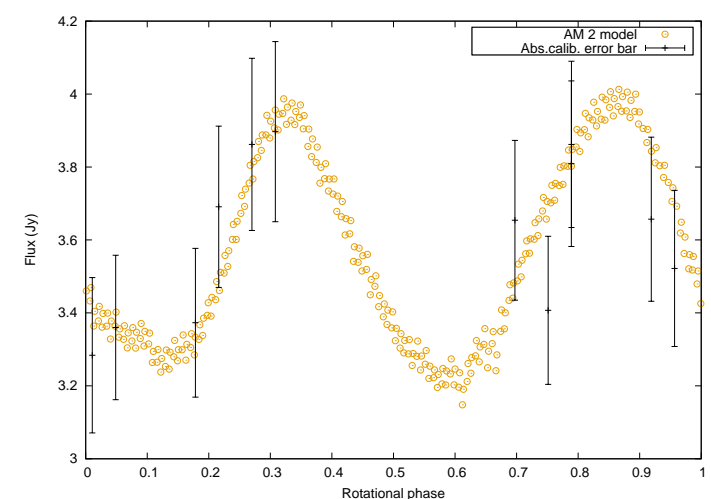

Fig. B.3. Asteroid (301) Bavaria AM 2 model thermal lightcurves and W4 data (see Table B.4).

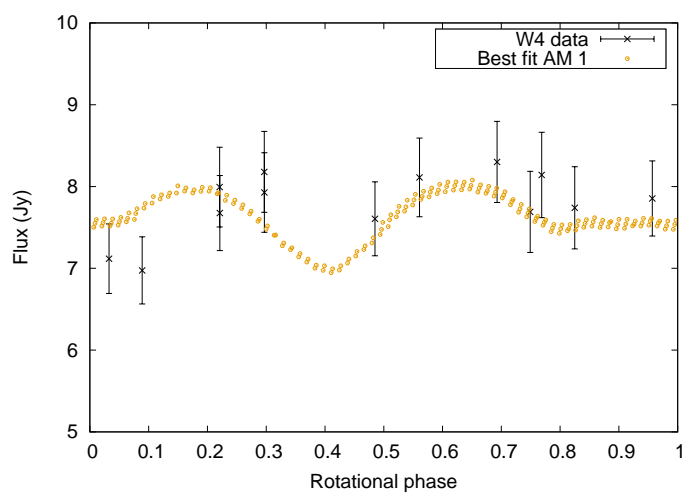

Fig. B.4. Asteroid (335) Roberta's WISE data and model thermal lightcurves for shape model 1 and the corresponding best-fitting solutions (very low thermal inertia of $15 \mathrm{SIu}$ ).

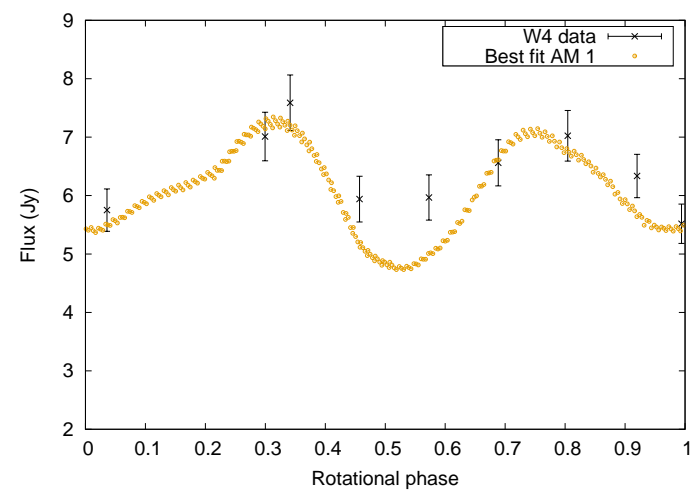

Fig. B.5. Asteroid (380) Fiducia's WISE data and model thermal lightcurves for shape model 1 and the corresponding best-fitting solutions (very low thermal inertia of 15 SIu; see Table B.6).

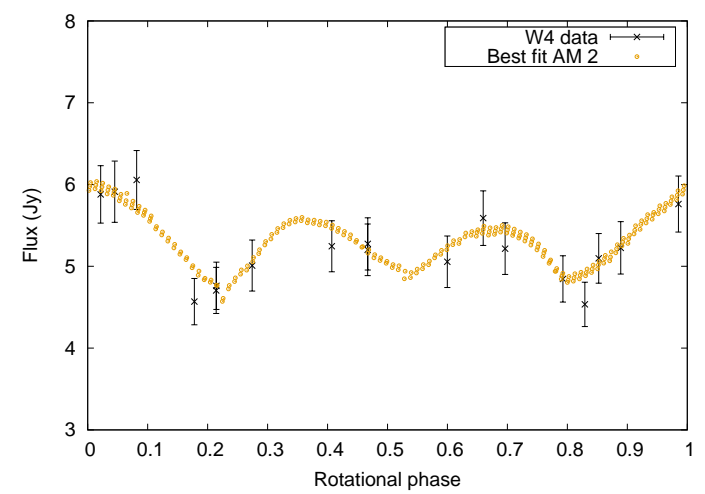

Fig. B.6. Asteroid (468) Lina's WISE data and model thermal lightcurves for shape model 2's corresponding best-fitting solutions (see Table B.7). 

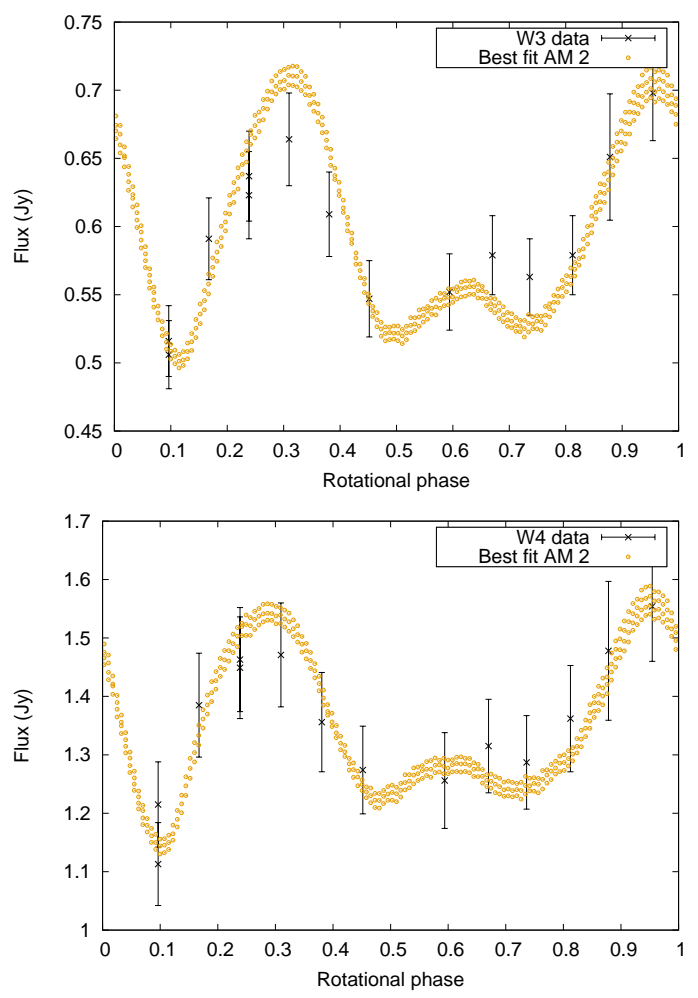

Fig. B.7. Asteroid (673) Edda's WISE data and model thermal lightcurves for shape model 2's best-fitting solution (very low thermal inertia of 3 SI units).
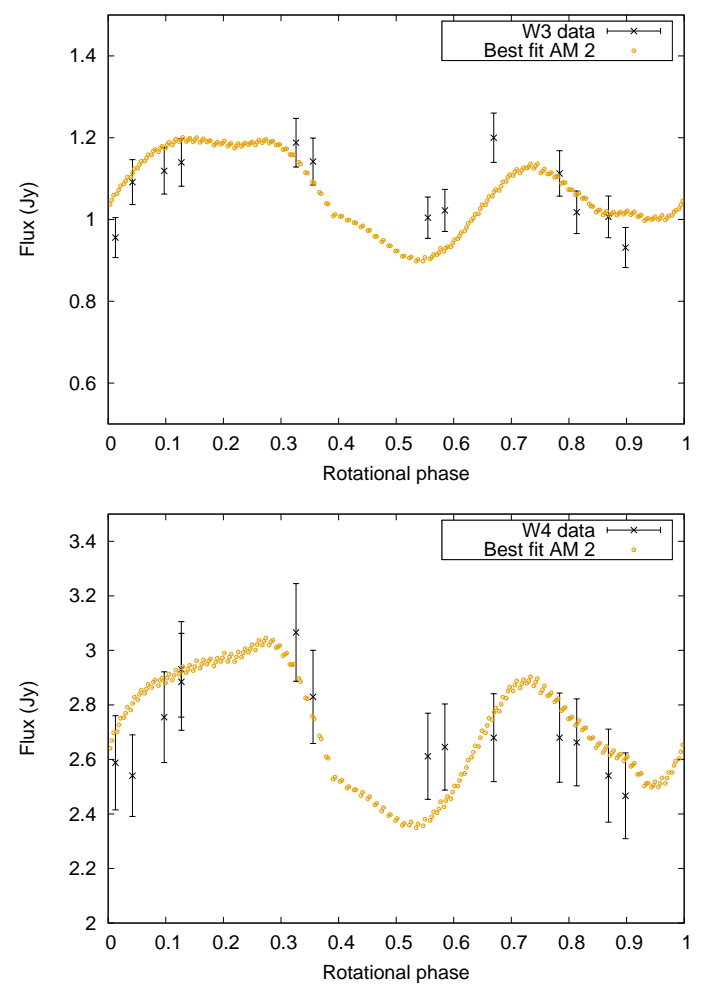

Fig. B.8. Asteroid (834) Burnhamia's WISE data and model thermal lightcurves for shape model 2's best-fitting solution (see Table B.11). 
A. Marciniak et al.: Thermal properties of slowly rotating asteroids


Fig. B.9. Asteroid (100) Hekate. Left, from top to bottom: observation to model ratios versus wavelength, heliocentric distance, rotational phase, and phase angle. Depending on the plot, the colour indicates the aspect angle or wavelength at which the observations were taken (see the legend on the right). The plots for AM 1 looked similar. Right: $\chi^{2}$ versus thermal inertia curves for (100) Hekate model 1 (top) and model 2 (bottom). In all the subsequent figures of this kind, the colours denote optimised diameter, while various symbols show various levels of surface roughness. 
A\&A 625, A139 (2019)
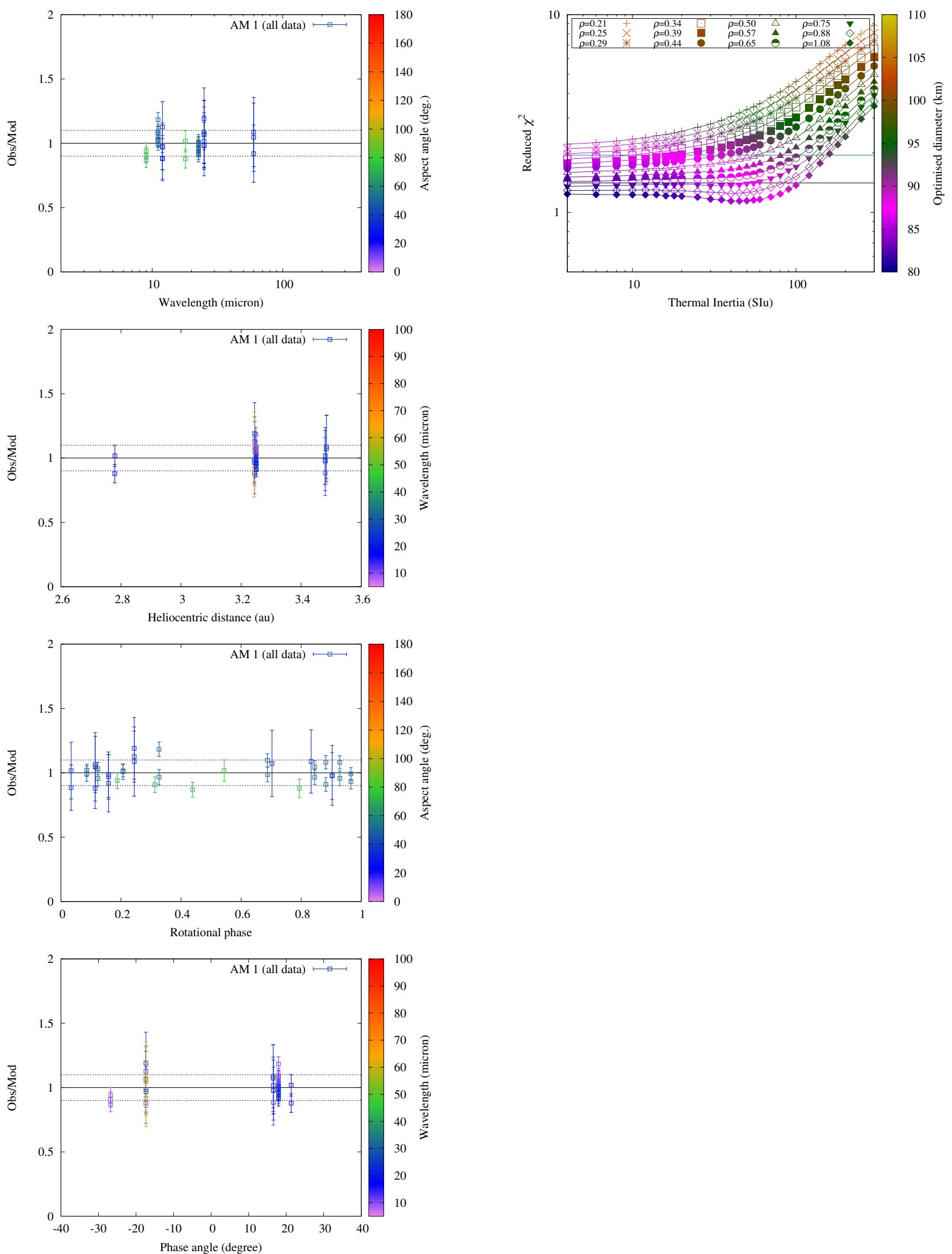

Fig. B.10. Asteroid (109) Felicitas. Left, from top to bottom: observation to model ratios versus wavelength, heliocentric distance, rotational phase, and phase angle for model AM 1 (AM 2 was rejected). Right: $\chi^{2}$ versus thermal inertia curves for model AM1. 
A. Marciniak et al.: Thermal properties of slowly rotating asteroids
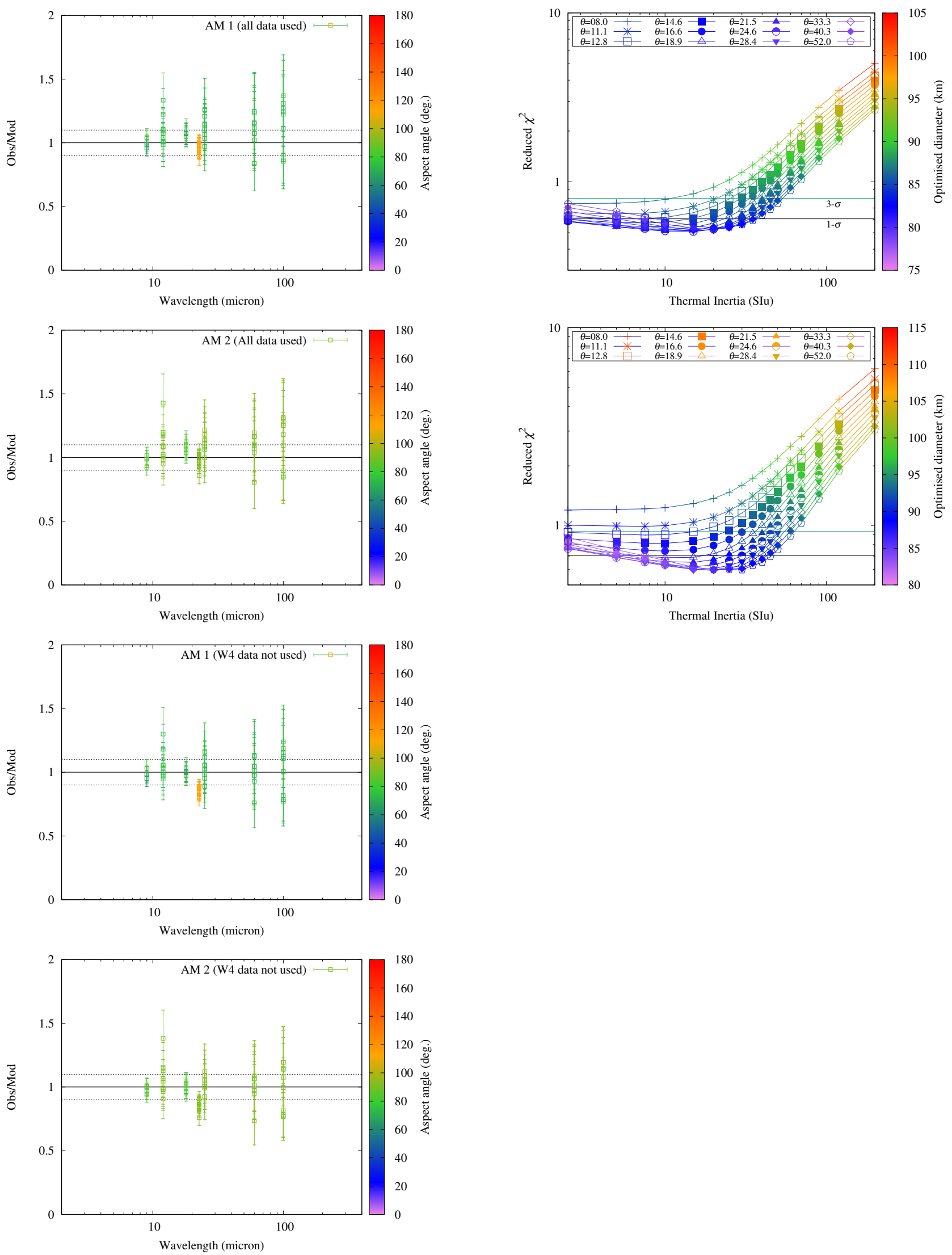

Fig. B.11. Asteroid (195) Eurykleia. Left: observation to model ratios versus wavelength for models AM 1 (first and third plot) and AM 2 (second and fourth) using all data to minimise the $\chi^{2}$ (top) and excluding the W4 data (bottom). The colour indicates the aspect angle at which the observations were taken. The AM 1 model's pole orientation is such that the WISE data would have been taken at a sub-observer latitude of $\sim 25^{\circ}$ south, and the rest of the data at $\sim 25^{\circ}$ north. On the other hand, if pole 2 is correct, then all data would have been taken at similar "equator-on" aspect angles between $80^{\circ}$ and $100^{\circ}$. Right: $\chi^{2}$ versus thermal inertia curves for model AM1 (top) and AM2 (bottom) using all thermal data. 

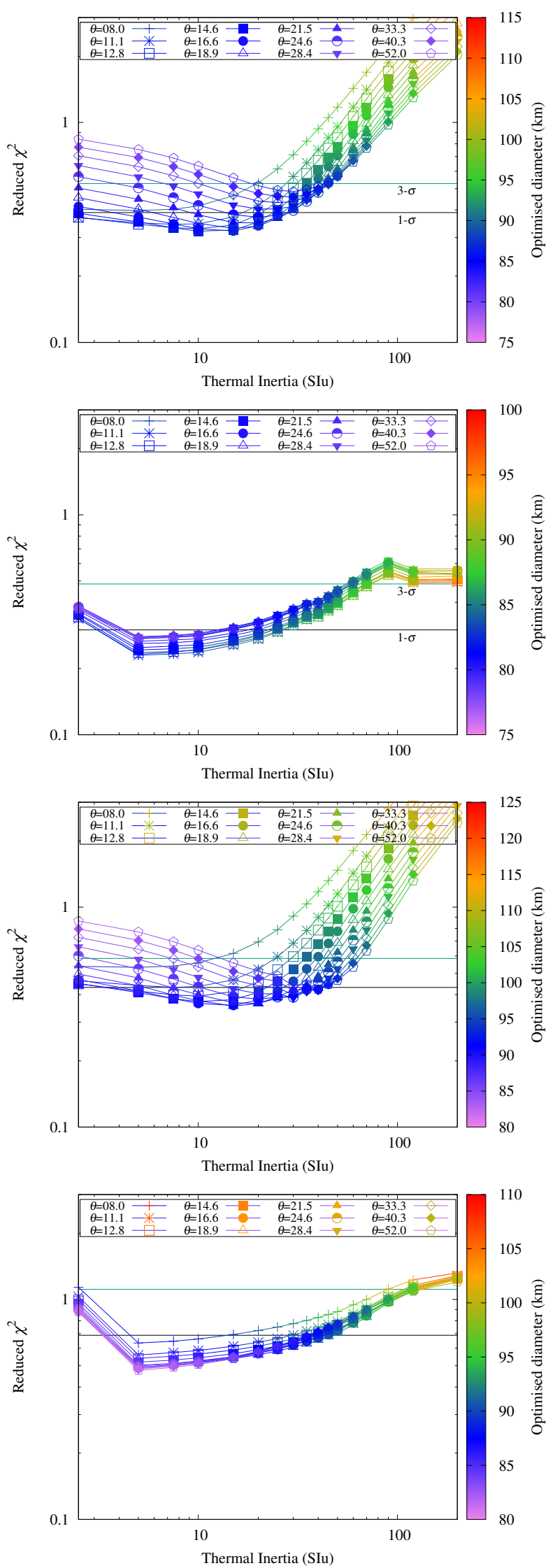

Fig. B.12. Additional $\chi^{2}$ versus thermal inertia plots for (195) Eurykleia. Top to bottom: model AM1 fitted to data excluding WISE W4 data, AM1 with W4 data only, model AM2 fitted to data excluding WISE W4 data, AM2 with W4 data only. 
A. Marciniak et al.: Thermal properties of slowly rotating asteroids
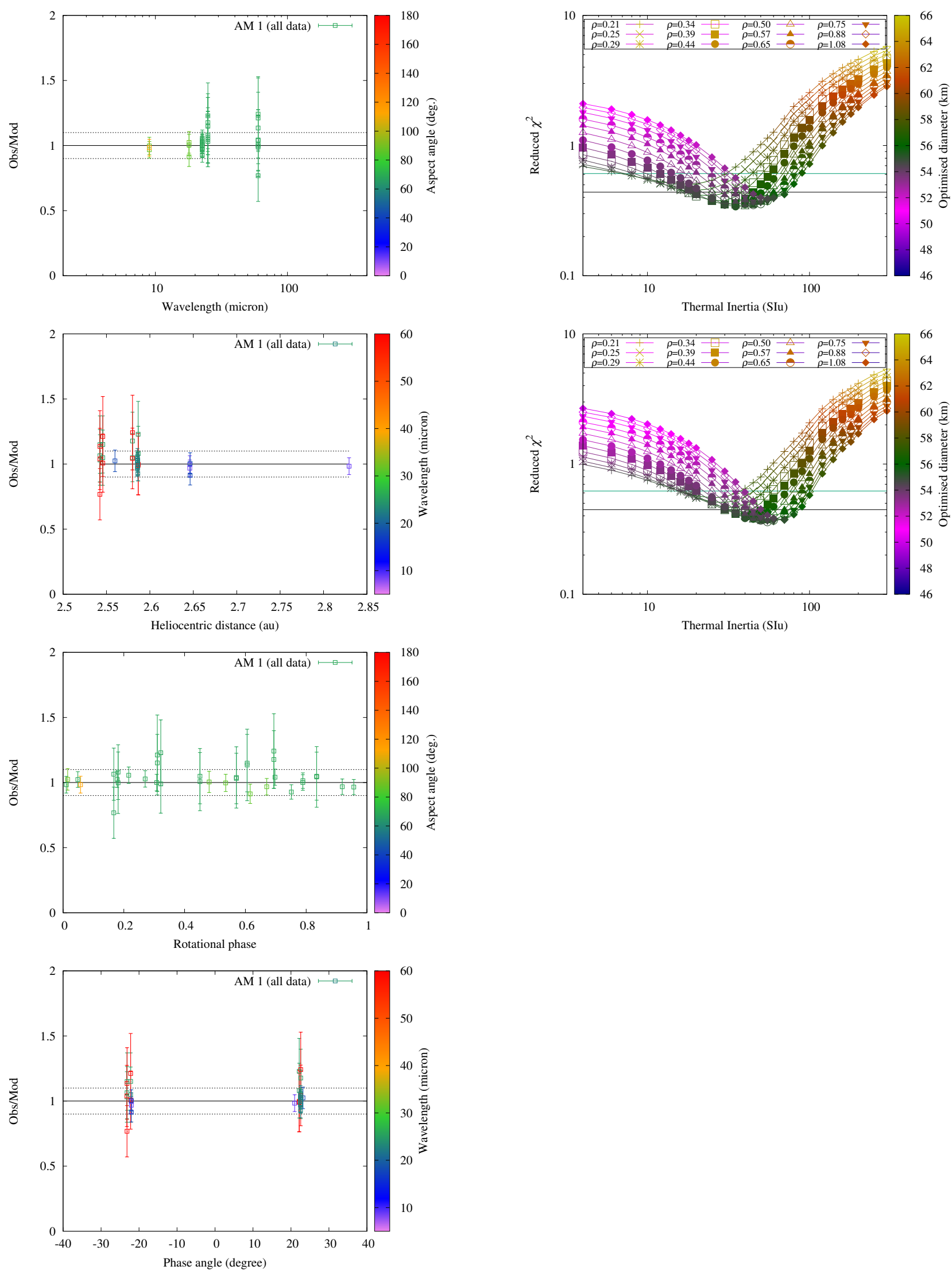

Fig. B.13. Asteroid (301) Bavaria. Left, from top to bottom: observation to model ratios versus wavelength, heliocentric distance, rotational phase, and phase angle for model AM 1 (the same plots for AM 2 looked similar). Right: $\chi^{2}$ versus thermal inertia curves for model AM1 (top), and AM2 (bottom). 

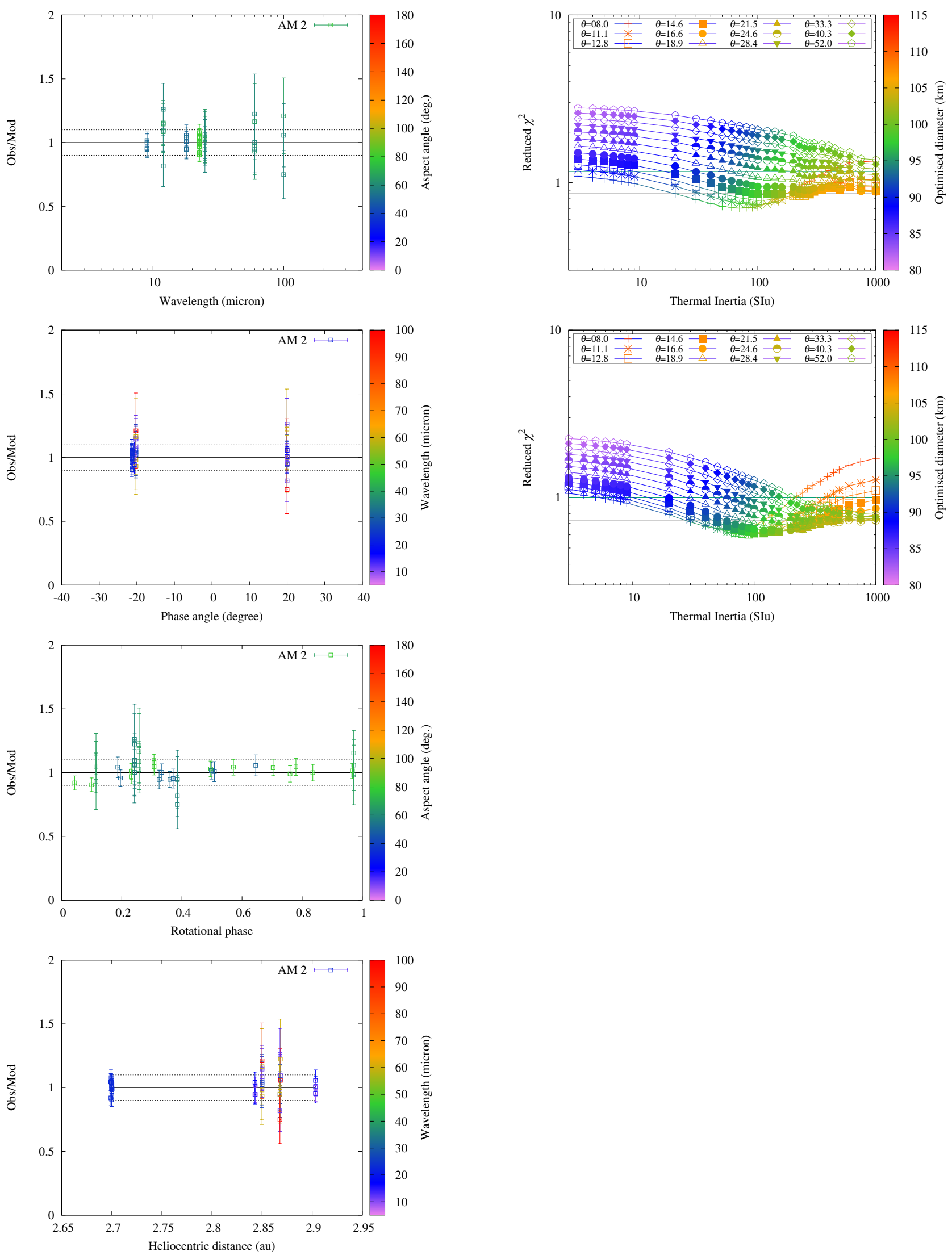

Fig. B.14. Asteroid (335) Roberta. Left: observation to model ratios versus wavelength, phase angle, rotational phase, and heliocentric distance for model AM 2. The plots for AM 1 looked similar. Right: $\chi^{2}$ versus thermal inertia curves for model AM1 (top), and AM2 (bottom). 
A. Marciniak et al.: Thermal properties of slowly rotating asteroids
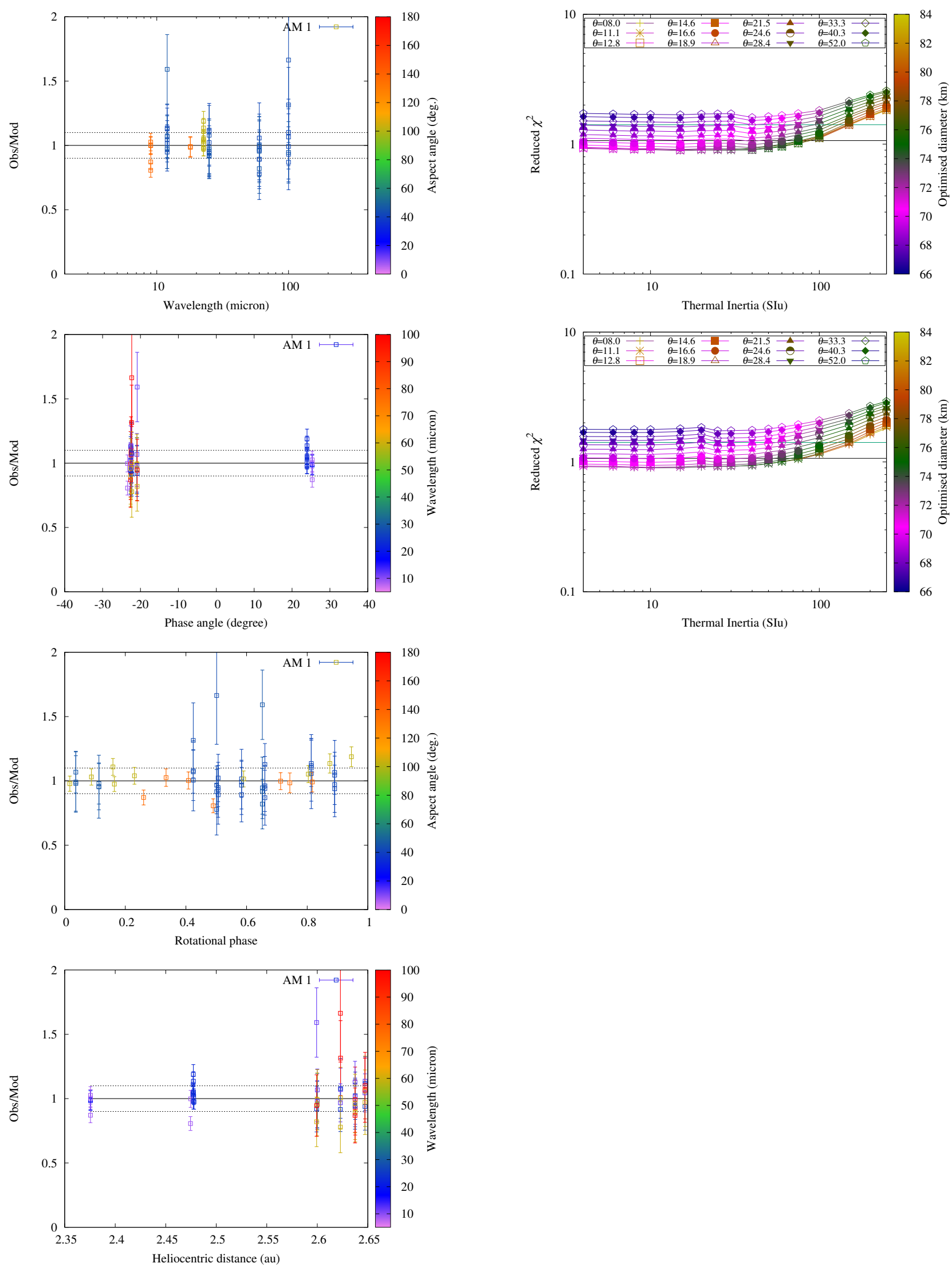

Fig. B.15. Asteroid (380) Fiducia. Left: observation to model ratios versus wavelength, phase angle, rotational phase, and heliocentric distance for model AM 1. The plots for AM 2 looked similar. Right: $\chi^{2}$ versus thermal inertia curves for model AM1 (top), and AM2 (bottom). 

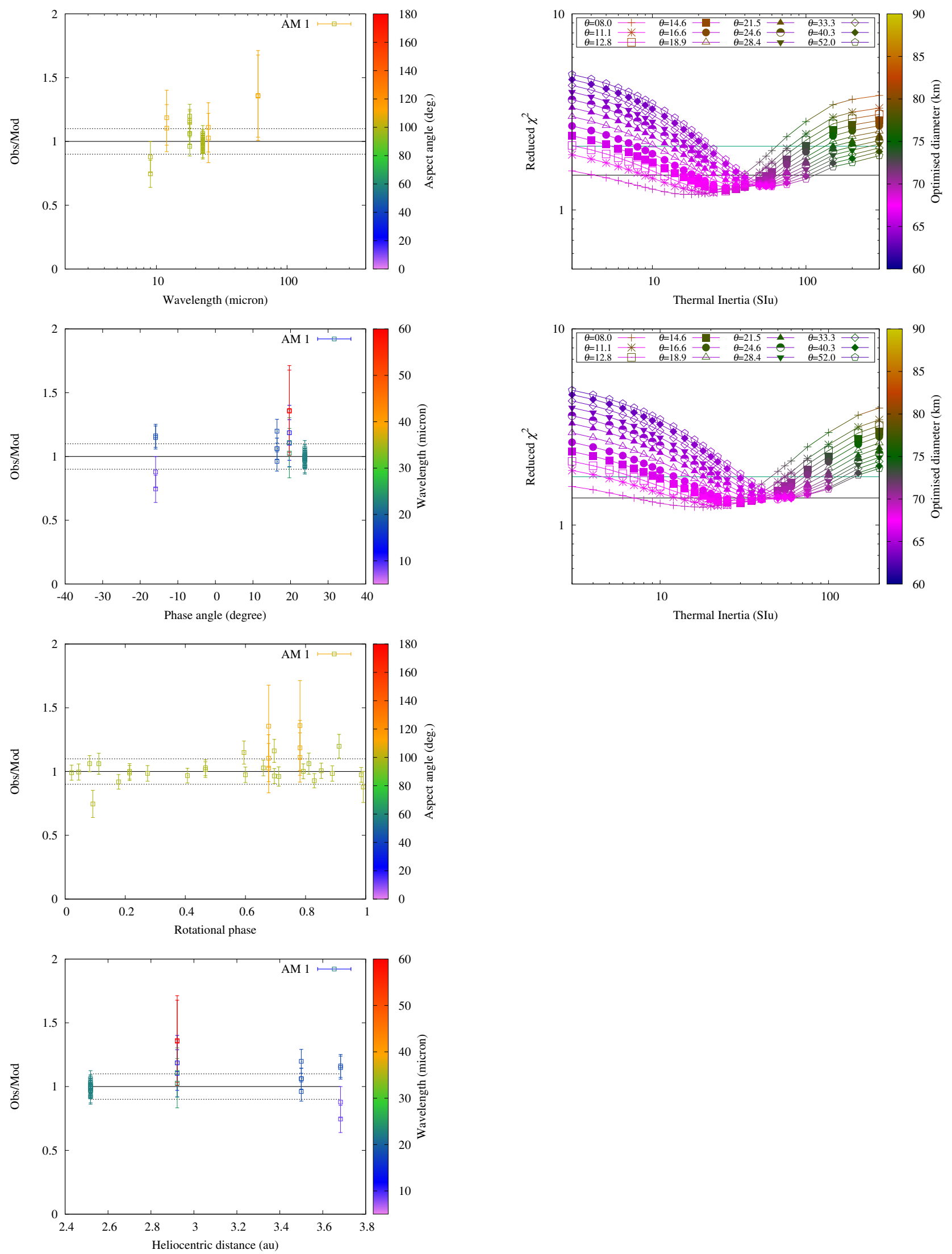

Fig. B.16. Asteroid (468) Lina. Left: observation to model ratios versus wavelength, phase angle, rotational phase, and heliocentric distance for model AM 1. The plots for AM 2 looked similar. Right: $\chi^{2}$ versus thermal inertia curves for model AM1 (top), and AM2 (bottom). 
A. Marciniak et al.: Thermal properties of slowly rotating asteroids
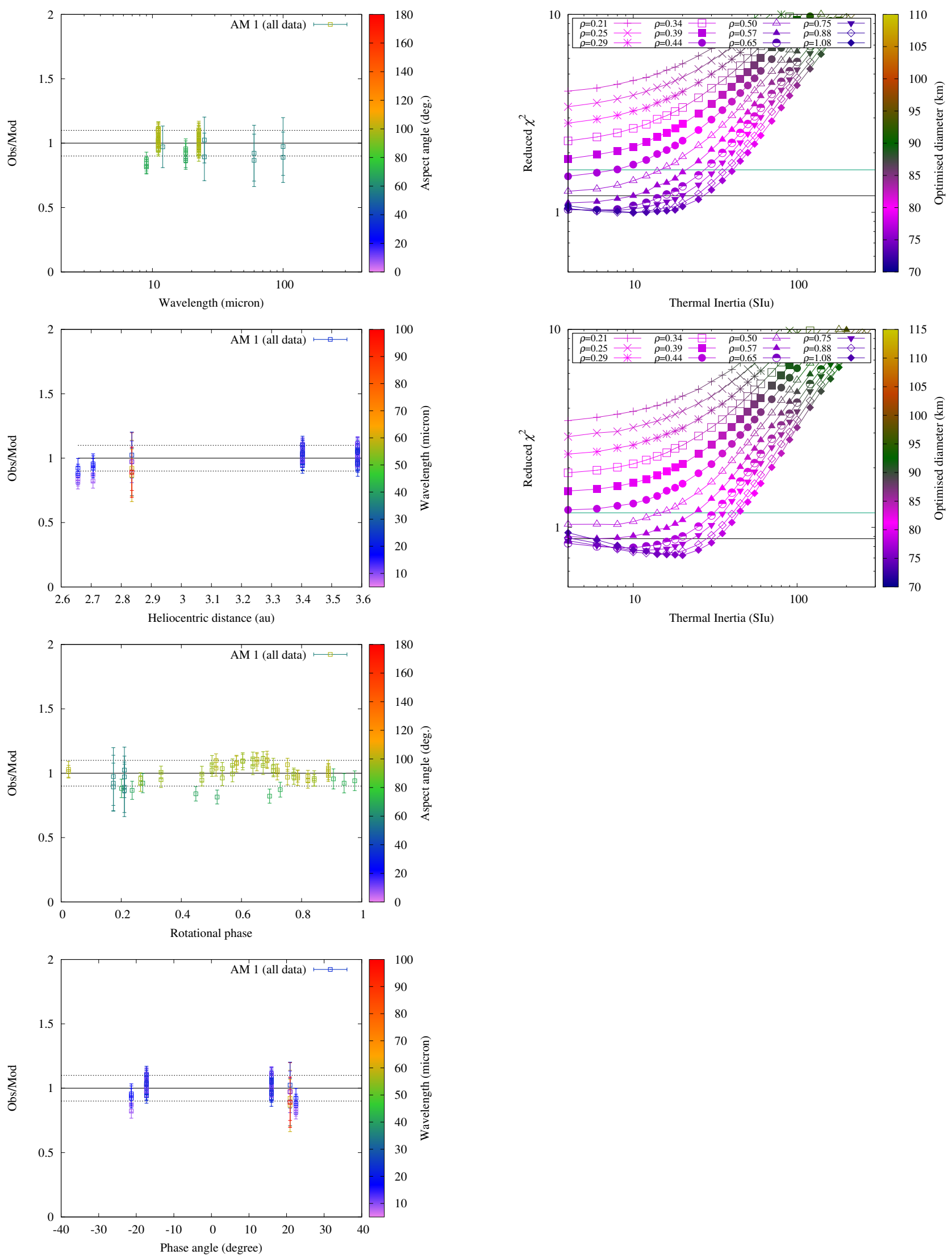

Fig. B.17. Asteroid (538) Friederike. From top to bottom: observation to model ratios versus wavelength, heliocentric distance, rotational phase, and phase angle for model AM 1 (the AM 2 model on all data did not provide an acceptable fit and was rejected). Right: $\chi^{2}$ versus thermal inertia curves for model AM1 (top), and AM2 (bottom) using WISE W3 and W4 data only. 

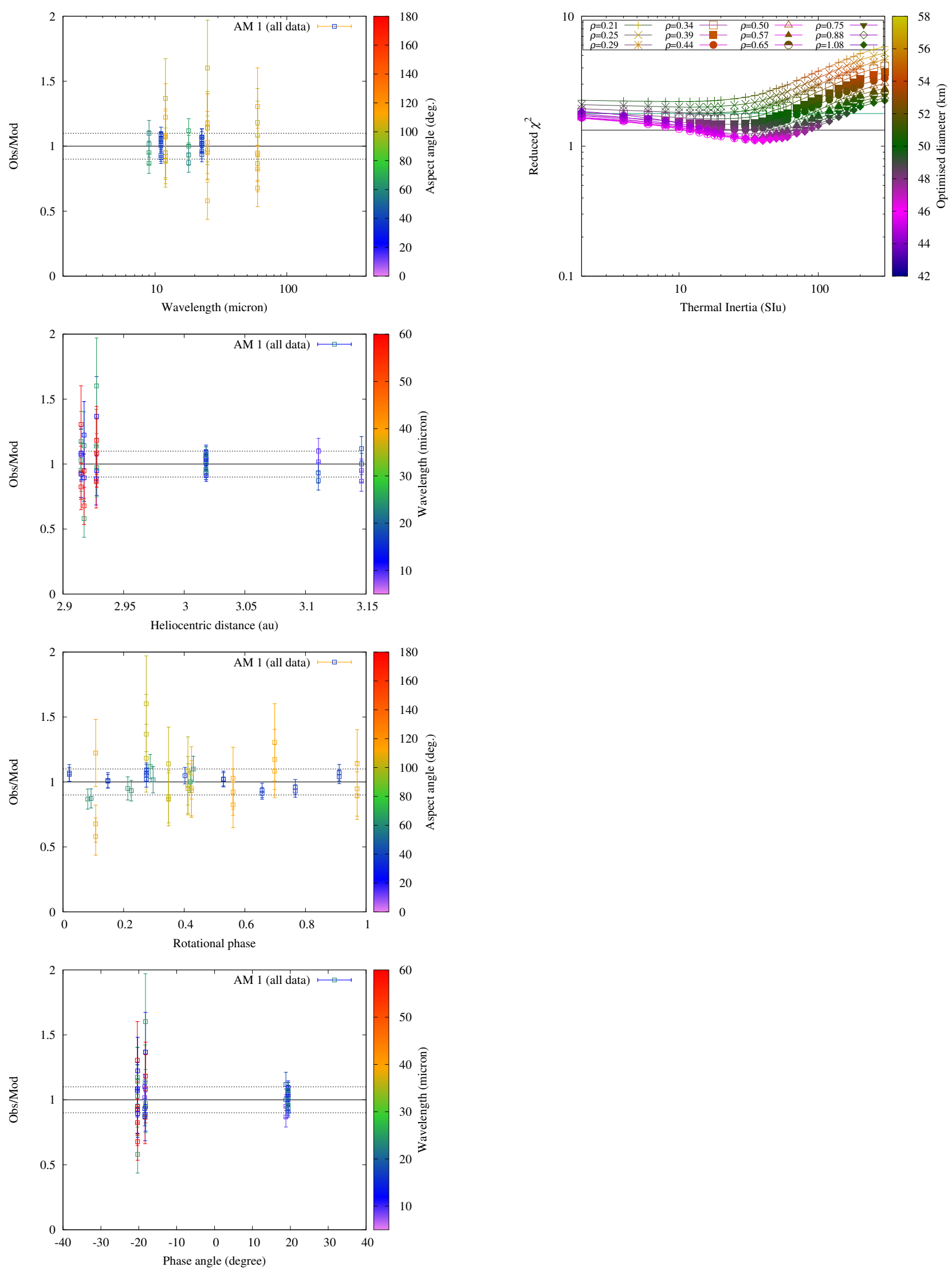

Fig. B.18. Asteroid (653) Berenike. Left, from top to bottom: observation to model ratios versus wavelength, heliocentric distance, rotational phase, and phase angle for model AM 1 (the AM 2 model did not provide an acceptable fit and was rejected). Right: $\chi^{2}$ versus thermal inertia curves for model AM1. 
A. Marciniak et al.: Thermal properties of slowly rotating asteroids
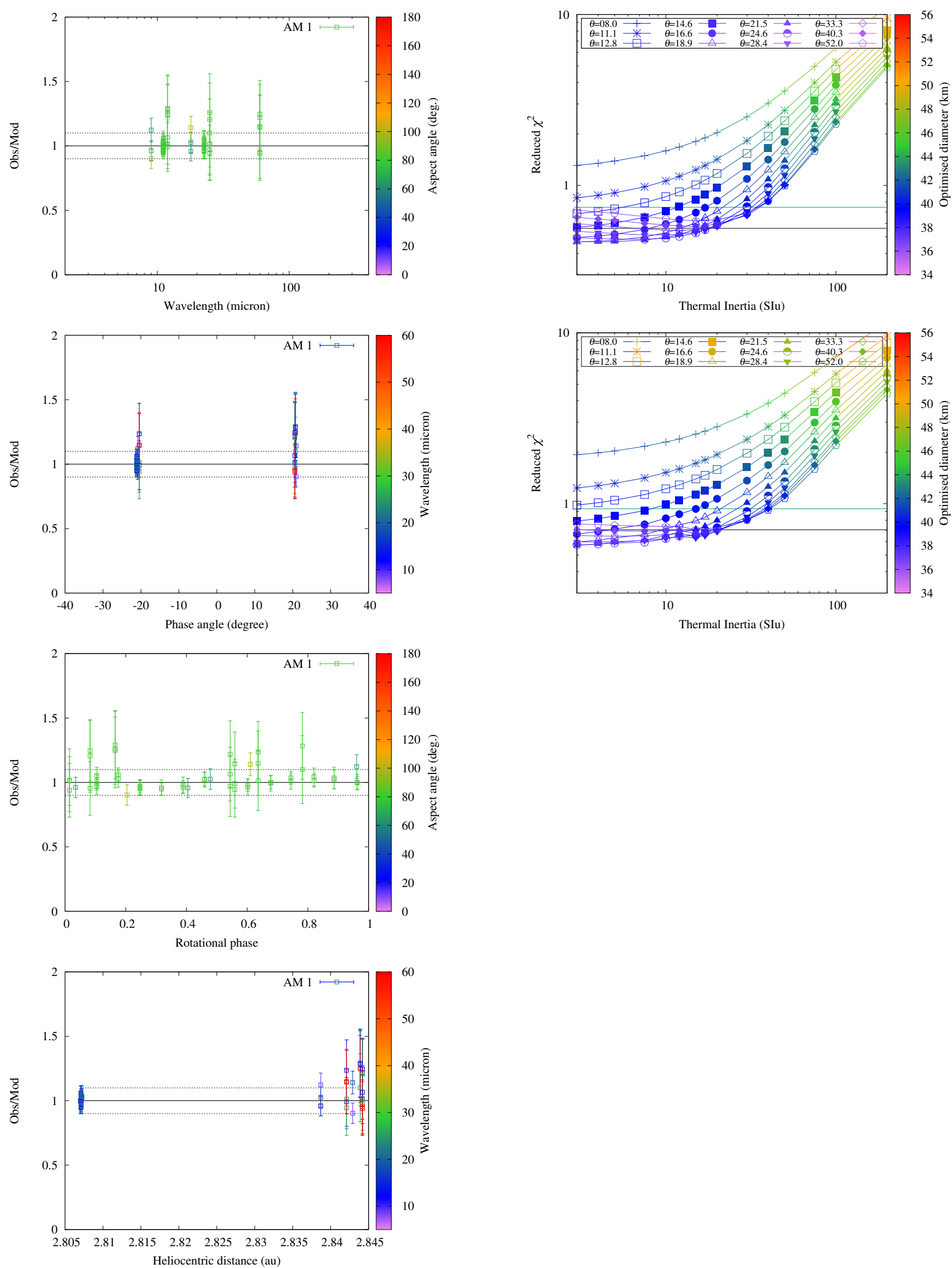

Fig. B.19. Asteroid (673) Edda. Left: observation to model ratios versus wavelength, phase angle, rotational phase, and heliocentric distance for model AM 1. The plots for AM 2 looked similar. Right: $\chi^{2}$ versus thermal inertia curves for model AM1 (top) and AM2 (bottom). 



Fig. B.20. Asteroid (834) Burnhamia. Left: observation to model ratios versus wavelength, phase angle, rotational phase, and heliocentric distance for model AM 1. The plots for AM 2 looked similar. Right: $\chi^{2}$ versus thermal inertia curves for model AM1 (top) and AM2 (bottom). 